\title{
Semilinear degenerate elliptic boundary value problems via the Semenov approximation
}

\author{
Kazuaki Taira \\ Dedicated to Professor Jerome Arthur Goldstein on the occasion of his 80th birthday
}

Received: date / Accepted: date

\begin{abstract}
This paper is devoted to the study of static bifurcation theory for a class of degenerate boundary value problems for semilinear elliptic differential operators of second-order which includes as particular cases the Dirichlet and Robin problems. The purpose of this paper is to generalize some results of Szulkin [52] to the degenerate case. We study the behavior of nontrivial solution branches for bounded nonlinear terms, and prove that the branches turn back towards the first eigenvalue of the linearized degenerate boundary value problem. Our proof is based on global static bifurcation theory for positive mappings in ordered Banach spaces, with the special emphasis on the Semenov approximation in Chemistry. The approach here is distinguished by the extensive use of the ideas and techniques characteristic of the recent developments in the $L^{p}$ theory of pseudo-differential operators.
\end{abstract}

Keywords Semilinear elliptic problem · degenerate Robin condition · global static bifurcation · simple eigenvalue - Leray-Schauder continuation method . Semenov approximation

Mathematics Subject Classification (2010) 35B32 35 J65 $\cdot 35$ P15 35 P30

\section{Contents}

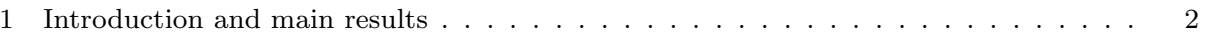

2 Linear elliptic boundary value problems . . . . . . . . . . . . . . . . . . . . . . . . . 12

3 Elements of topological methods in nonlinear analysis . . . . . . . . . . . . . . . . . 29

Proof of Theorem 1.2 . . . . . . . . . . . . . . . . . . . . . . . . 38

5 The Lyapunov-Schmidt procedure in the Banach space $C(\bar{D})$. . . . . . . . . . . . . 51

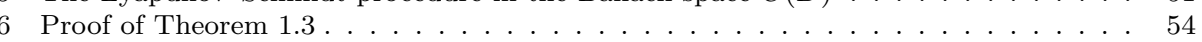

7 Proof of Theorem 1.4 . . . . . . . . . . . . . . . . . . . . . . . . . . . . . . 62

8 The Leray-Schauder index of critical points and its application . . . . . . . . . . . . 68

9 Concluding remarks . . . . . . . . . . . . . . . . . . . . . . . . . . . . . . . . . . 76

10 Appendix: The maximum principle in $L^{p}$ Sobolev spaces . . . . . . . . . . . . . . . . 76

Kazuaki Taira

Institute of Mathematics, University of Tsukuba, Tsukuba 305-8571, Japan

E-mail: taira@math.tsukuba.ac.jp 


\section{Introduction and main results}

Let $D$ be a bounded domain of Euclidean space $\mathbf{R}^{N}, N \geq 2$, with $C^{\infty}$ boundary $\partial D$; its closure $\bar{D}=D \cup \partial D$ is an $N$-dimensional, compact $C^{\infty}$ manifold with boundary. In this paper we consider a second-order, uniformly elliptic differential operator with real $C^{\infty}$ coefficients in divergence form

$$
A u(x)=-\sum_{i=1}^{N} \frac{\partial}{\partial x_{i}}\left(\sum_{j=1}^{N} a^{i j}(x) \frac{\partial u}{\partial x_{j}}(x)\right)+c(x) u(x) .
$$

Here:

(1) $a^{i j}(x)=a^{j i}(x)$ for all $x \in \bar{D}$ and $1 \leq i, j \leq N$, and there exists a constant $a_{0}>0$ such that

$$
\sum_{i, j=1}^{N} a^{i j}(x) \xi_{i} \xi_{j} \geq a_{0}|\xi|^{2} \quad \text { for all } x \in \bar{D} \text { and } \xi \in \mathbf{R}^{N}
$$

(2) $c(x) \geq 0$ on $\bar{D}$.

We study the following linear elliptic boundary value problem: Given function $f$ defined in $D$, find a function $u$ in $D$ such that

$$
\begin{cases}A u=f & \text { in } D, \\ B u=a\left(x^{\prime}\right) \frac{\partial u}{\partial \boldsymbol{\nu}}+b\left(x^{\prime}\right) u=0 & \text { on } \partial D .\end{cases}
$$

Here:

(3) $a \in C^{\infty}(\partial D)$ and $a\left(x^{\prime}\right) \geq 0$ on $\partial D$

(4) $b \in C^{\infty}(\partial D)$ and $b\left(x^{\prime}\right) \geq 0$ on $\partial D$.

(5) $\partial / \partial \boldsymbol{\nu}$ is the outward conormal derivative associated with the operator $A$ :

$$
\frac{\partial}{\partial \boldsymbol{\nu}}=\sum_{i, j=1}^{N} a^{i j} n_{j} \frac{\partial}{\partial x_{i}},
$$

where $\mathbf{n}=\left(n_{1}, n_{2}, \ldots, n_{N}\right)$ is the unit outward normal to the boundary $\partial D$ (see Figure 1.1).

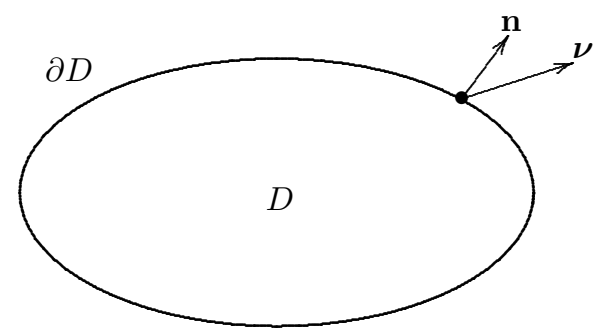

Fig. 1.1 The unit outward normal $\mathbf{n}$ and the conormal $\boldsymbol{\nu}$ to $\partial D$ 
Remark 1.1 The terms $a\left(x^{\prime}\right) \partial u / \partial \boldsymbol{\nu}$ and $b\left(x^{\prime}\right) u$ of the boundary condition $B$ are supposed to correspond to reflection and absorption phenomena at the boundary $\partial D$, respectively (see [64]). The situation may be represented schematically as in Figure 1.2.
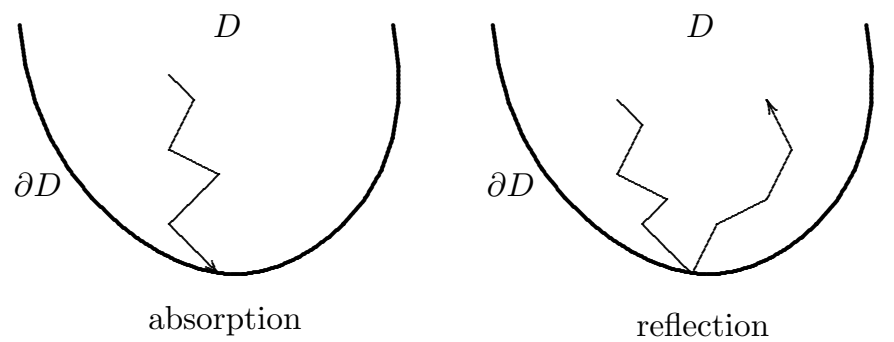

Fig. 1.2 The absorption phenomenon and the reflection phenomenon

It is worth pointing out (see [2, Definition 10.1], [35, Theorem 5]) that the elliptic boundary value problem (1.1) is coercive (or non-degenerate) if and only if either $a\left(x^{\prime}\right)>0$ on $\partial D$ or $a\left(x^{\prime}\right) \equiv 0$ and $b\left(x^{\prime}\right)>0$ on $\partial D$. In particular, if $a\left(x^{\prime}\right) \equiv 1$ and $b\left(x^{\prime}\right) \equiv 0$ on $\partial D$ (resp. $a\left(x^{\prime}\right) \equiv 0$ and $b\left(x^{\prime}\right) \equiv 1$ on $\left.\partial D\right)$, then the boundary condition $B$ is the Neumann (resp. Dirichlet) condition.

However, our boundary condition $B$ is degenerate in the Lopatinski-Shapiro sense (see [3, p. 633], [24, Chapitre V, condition (4.5)], [31, Chapter XX, Definition 20.1.1], [73, Chapter II, Condition 11.1]). This is due to the fact that the so-called Lopatinski-Shapiro complementary condition is violated at each point of the set

$$
M=\left\{x^{\prime} \in \partial D: a\left(x^{\prime}\right)=0\right\}
$$

(see [60, Section 6.6]).

The present paper is amply illustrated; 1 table and 21 figures are provided with appropriate captions in such a fashion that a broad spectrum of readers could understand our problem and main results.

\subsection{The closed realization $\mathfrak{A}$}

First, we study problem (1.1) in the framework of $L^{2}$ spaces. To do this, we associate with problem (1.1) a unbounded linear operator $\mathfrak{A}$ from the Hilbert space $L^{2}(D)$ into itself as follows:

(a) The domain of definition $D(\mathfrak{A})$ of $\mathfrak{A}$ is the space

$$
D(\mathfrak{A})=\left\{u \in H^{2}(D)=W^{2,2}(D): B u=0 \text { on } \partial D\right\} .
$$

(b) $\mathfrak{A} u=A u$ for every $u \in D(\mathfrak{A})$.

Our starting point is the following theorem (see Theorem 2.1):

Theorem 1.1 Assume that the following hypotheses (H.1) and (H.2) are satisfied: 
(H.1) $a\left(x^{\prime}\right) \geq 0$ and $b\left(x^{\prime}\right) \geq 0$ on $\partial D$.

(H.2) $a\left(x^{\prime}\right)+b\left(x^{\prime}\right)>0$ on $\partial D$.

Then the operator $\mathfrak{A}$ is a non-negative, selfadjoint operator in the space $L^{2}(D)$. Moreover, the spectrum of $\mathfrak{A}$ is discrete and the eigenvalues $\lambda_{j}$ of $\mathfrak{A}$ have finite multiplicities. In particular, the first eigenvalue $\lambda_{1}$ of $\mathfrak{A}$ is positive and algebraically simple, that is, $0<\lambda_{1}<\lambda_{2}$, and the associated eigenfunction $\varphi_{1} \in D(\mathfrak{A})$ is strictly positive everywhere in $D$ :

$$
\begin{cases}A \varphi_{1}=\lambda_{1} \varphi_{1} & \text { in } L^{2}(D) \\ B \varphi_{1}=0 & \text { on } \partial D \\ \varphi_{1}>0 & \text { in } D\end{cases}
$$

Remark 1.2 A probabilistic meaning of condition (H.2) is that absorption phenomenon occurs at each point of the boundary portion

$$
M=\left\{x^{\prime} \in \partial D: a\left(x^{\prime}\right)=0\right\},
$$

while reflection phenomenon occurs at each point of the boundary portion

$$
\partial D \backslash M=\left\{x^{\prime} \in \partial D: a\left(x^{\prime}\right)>0\right\} .
$$

More precisely, a Markovian particle moves continuously in the state space $\bar{D} \backslash M$ until it "dies" at the time when it reaches the set $M$ where the particle is definitely absorbed (see Figure1.3).

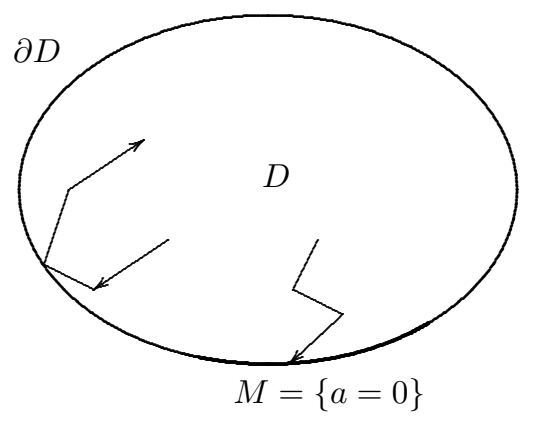

Fig. 1.3 A probabilistic meaning of condition (H.2)

We give a simple example of eigenvalue problems for the operator $\mathfrak{A}$ in the plane $\mathbf{R}^{2}$ ([66, Theorem 2.3] and [32, Theorem II, formula (3)]):

Example 1.1 Let $D=\left\{\left(x_{1}, x_{2}\right) \in \mathbf{R}^{2}: x_{1}^{2}+x_{2}^{2}<1\right\}$ be the unit disk with the boundary (unit circle) $S=\left\{\left(x_{1}, x_{2}\right) \in \mathbf{R}^{2}: x_{1}^{2}+x_{2}^{2}=1\right\}$. For a local coordinate system $x_{1}=\cos \theta, x_{2}=\sin \theta$ with $\theta \in[0,2 \pi]$ on $S$, we define a function $a\left(x_{1}, x_{2}\right)$ as follows:

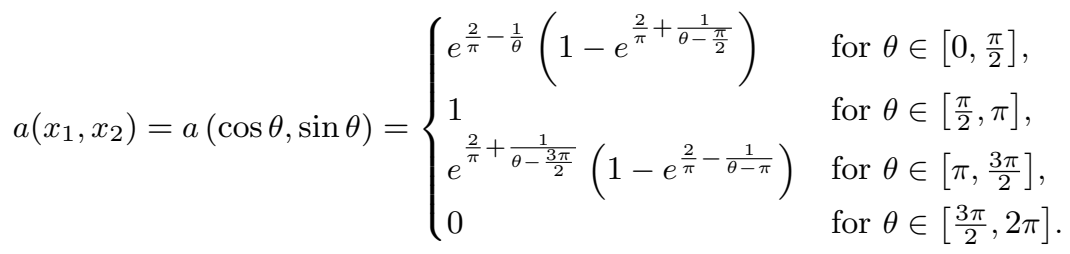


We consider the negative Laplacian $-\Delta_{B}$ with the degenerate Robin boundary condition $B$ in the unit disk $D$ :

$$
\begin{cases}-\Delta u=\lambda u & \text { in } D, \\ B u=a\left(x_{1}, x_{2}\right) \frac{\partial u}{\partial \mathbf{n}}+\left(1-a\left(x_{1}, x_{2}\right)\right) u=0 & \text { on } S .\end{cases}
$$

Then we have the asymptotic expansion formula for the $\Theta$-function

$$
\Theta(t)=\sum_{j=1}^{\infty} \exp \left[-\lambda_{j} t\right]=\frac{1}{4 t}+\frac{\sqrt{\pi}}{8 \sqrt{t}}+o\left(\frac{1}{\sqrt{t}}\right) \quad \text { as } t \downarrow 0 .
$$

1.2 Bifurcation theory for the semilinear elliptic boundary value problem

Now, as an application of Theorem 1.1 we consider global static bifurcation problems for the following semilinear elliptic boundary value problem: Let $h(t)$ be a real-valued function on $\mathbf{R}$, not depending explicitly on $x$. For a real parameter $\lambda$, find a function $u$ defined on $\bar{D}$ such that

$$
\begin{cases}A u-\lambda u+f(u)=0 & \text { in } D, \\ B u=a\left(x^{\prime}\right) \frac{\partial u}{\partial \boldsymbol{\nu}}+b\left(x^{\prime}\right) u=0 & \text { on } \partial D .\end{cases}
$$

A solution $u \in C^{2}(\bar{D})$ of the semilinear problem (1.2) is said to be nontrivial if it does not identically equal to zero on $\bar{D}$. We call a nontrivial solution $u$ of the semilinear problem (1.2) a positive solution (resp. negative solution) if $u(x) \geq 0$ (resp. $u(x) \leq 0$ ) on $\bar{D}$.

If the boundary condition $B$ is non-degenerate such as the Dirichlet, Neumann and Robin boundary conditions, then the exact number of solutions of the semilinear elliptic boundary value problem (1.2) was studied by Ambrosetti-Prodi [12], Amann [4], [6], Ambrosetti-Mancini [11], Berger [18], Berestycki [16] and Szulkin [52]. See also Ambrosetti-Prodi [13], Runst-Sickel [49], Chang [23], Drábek-Milota [29] and the references therein.

This paper is a continuation of the previous papers [57] through [62] and [70] in the case where the boundary condition $B$ is degenerate as in Example 1.1.

By using the bifurcation theory from a simple eigenvalue due to CrandallRabinowitz [26] (Theorem 3.3), we can prove that there exist precisely two nontrivial branches of solutions of the semilinear problem (1.2) bifurcating at the point $\left(\lambda_{1}, 0\right)$ where $\lambda_{1}$ is the first eigenvalue of $\mathfrak{A}$ (cf. [55, Theorem 3]). Theorems 1.3 and 1.4 below characterize them globally.

It is worthwhile pointing out here that the bifurcation solution curve $(\lambda, u)$ of the semilinear problem (1.2) is "formally" given by the so-called Semenov approximation in Chemistry ([51])

$$
\lambda=\lambda_{1}+\frac{f(u)}{u} .
$$

Indeed, Theorem 1.1 asserts that the first eigenvalue $\lambda_{1}$ is the unique eigenvalue corresponding to a positive eigenfunction of the operator $\mathfrak{A}$. Hence, if we rewrite the semilinear problem (1.2) as

$$
\left\{\begin{array}{l}
\mathfrak{A} u=\lambda u-f(u)=\left(\lambda-\frac{f(u)}{u}\right) u, \\
u>0 \text { in } D
\end{array}\right.
$$


then we have the equation

$$
\lambda_{1}=\lambda-\frac{f(u)}{u} .
$$

This proves the desired formula (1.3) (see Figure 1.4).

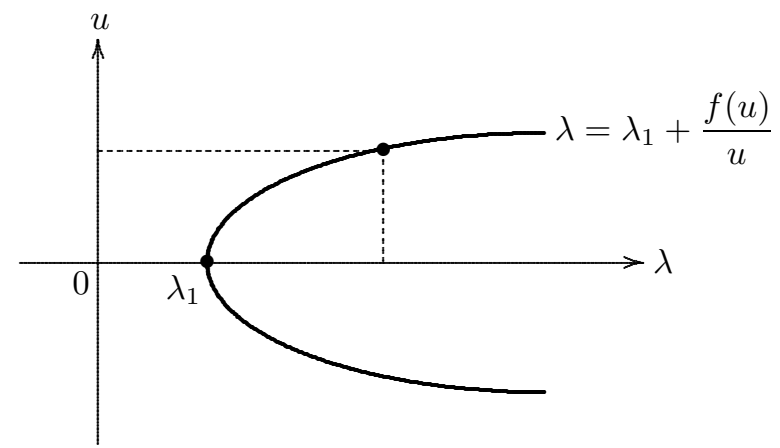

Fig. 1.4 The formal positive solution curve $\lambda=\lambda_{1}+\frac{f(u)}{u}$

(I) The asymptotically linear nonlinearity case: The first theorem is a generalization of Szulkin [52, Theorem 1.3] to the degenerate case:

Theorem 1.2 Assume that hypotheses (H.1) and (H.2) are satisfied and let $\lambda_{1}$ be the first eigenvalue of $\mathfrak{A}$. Let $f(t)$ be a function of class $C^{1}$ on $\mathbf{R}$ such that

$$
f(0)=f^{\prime}(0)=0 .
$$

Moreover, we assume that

$$
\sup _{t \in \mathbf{R}}\left|f^{\prime}(t)\right|<\lambda_{1}-\delta \quad \text { for some constant } 0<\delta<\lambda_{1},
$$

and further that the derivative $f^{\prime}(t)$ is strictly decreasing for $t<0$ and strictly increasing for $t>0$ and that there exist constants $k_{-}>0$ and $k_{+}>0$ such that

$$
\begin{aligned}
& \lim _{t \rightarrow-\infty} f^{\prime}(t)=k_{-}, \\
& \lim _{t \rightarrow+\infty} f^{\prime}(t)=k_{+} .
\end{aligned}
$$

Then we have the following four assertions (see Figures 1.5 and 1.6):

(i) The point $\left(\lambda_{1}, 0\right)$ is a bifurcation point of the semilinear problem (1.2). More precisely, the set of nontrivial solutions of the semilinear problem (1.2) consists of two $C^{1}$ curves $\Gamma_{-}$and $\Gamma_{+}$parametrized respectively by $\lambda$ as follows:

$$
\begin{aligned}
& \Gamma_{-}=\left\{\left(\lambda, u_{-}(\lambda)\right) \in \mathbf{R} \times C(\bar{D}): \lambda_{1} \leq \lambda<\lambda_{1}+k_{-}\right\}, \\
& \Gamma_{+}=\left\{\left(\lambda, u_{+}(\lambda)\right) \in \mathbf{R} \times C(\bar{D}): \lambda_{1} \leq \lambda<\lambda_{1}+k_{+}\right\} .
\end{aligned}
$$

The branch $\Gamma_{-}$is negative and the branch $\Gamma_{+}$is positive except at $\left(\lambda_{1}, 0\right)$. 
(ii) The uniform norms $\left\|u_{-}(\lambda)\right\|$ and $\left\|u_{+}(\lambda)\right\|$ tend to $\infty$ as $\lambda \rightarrow \lambda_{1}+k_{-}$and as $\lambda \rightarrow \lambda_{1}+k_{+}$, respectively. In other words, $\lambda_{1}+k_{+}$is a bifurcation point from infinity for positive solutions and $\lambda_{1}+k_{-}$is a bifurcation point from infinity for negative solutions, respectively.

(iii) The semilinear problem (1.2) has no other positive or negative solutions for any $\lambda \geq \lambda_{1}$.

(iv) The semilinear problem (1.2) has no other nontrivial solutions for $\lambda \in\left[\lambda_{1}, \lambda_{2}\right]$.

Remark 1.3 If either $c_{1}:=\lambda_{1}+k_{+}>\lambda_{2}$ or $c_{2}:=\lambda_{1}+k_{-}>\lambda_{2}$, then we do not describe the behaviors of $\Gamma_{\mp}$ for $\lambda \geq \lambda_{2}$.

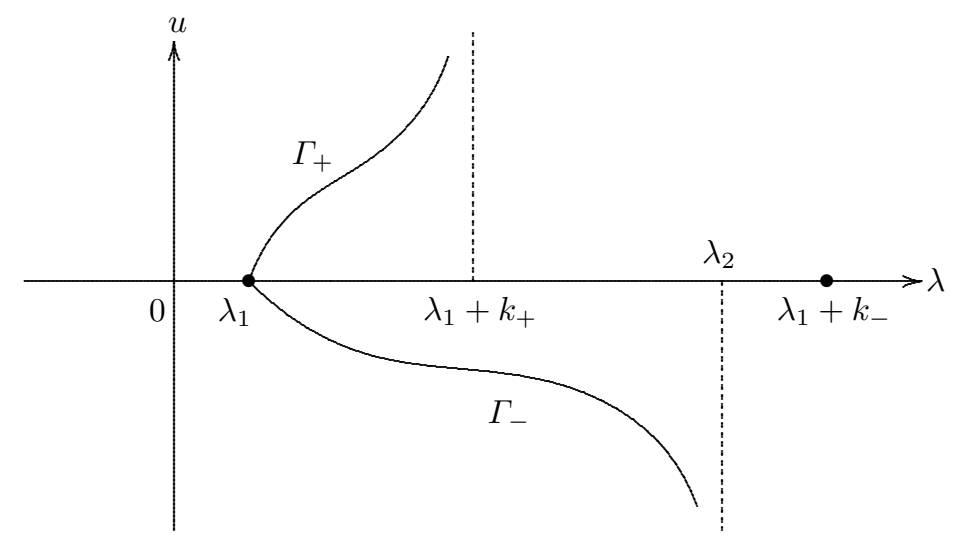

Fig. 1.5 The bifurcation curves $\Gamma_{+}$and $\Gamma_{-}$in the case $c_{1}=\lambda_{1}+k_{+}<\min \left\{c_{2}, \lambda_{2}\right\}=\lambda_{2}$

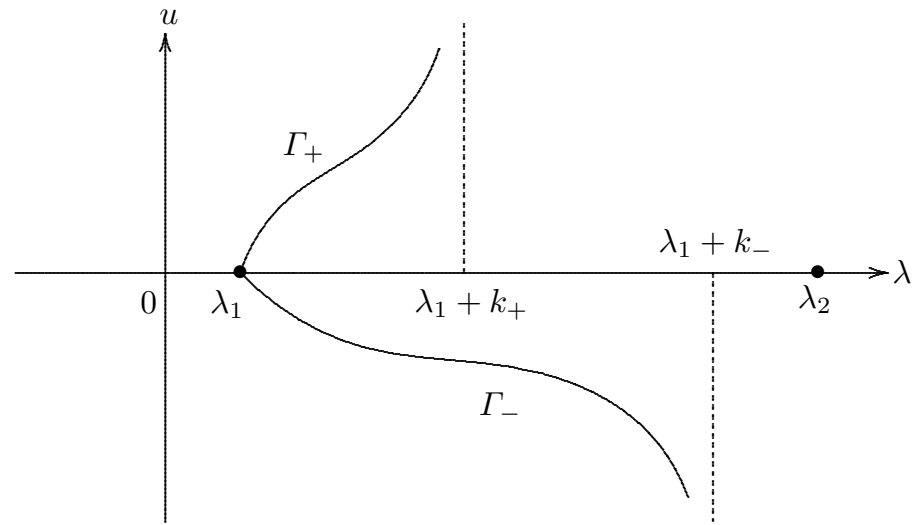

Fig. 1.6 The bifurcation curves $\Gamma_{+}$and $\Gamma_{-}$in the case $c_{2}=\lambda_{1}+k_{-} \leq \lambda_{2}$

For Theorem 1.2, we give a simple example of the function $f(t)$ : 
Example 1.2 If $k_{ \pm}$are constants such that

$$
0<k_{ \pm}<\lambda_{1}-\delta \quad \text { for some constant } \delta>0
$$

then we let

$$
f(t):= \begin{cases}k_{+}\left(t+\frac{1}{2 t}-\frac{4}{3}\right) & \text { for } t>1 \\ \frac{k_{+}}{6} t^{3} & \text { for } 0 \leq t \leq 1 \\ \frac{k_{-}}{6} t^{3} & \text { for }-1 \leq t \leq 0 \\ k_{-}\left(t+\frac{1}{2 t}+\frac{4}{3}\right) & \text { for } t<-1\end{cases}
$$

The next corollary is a generalization of Szulkin [52, Corollary 1.4] to the degenerate case:

Corollary 1.1 We let

$$
\begin{aligned}
& c_{1}:=\min \left\{\lambda_{1}+k_{-}, \lambda_{1}+k_{+}\right\}, \\
& c_{2}:=\max \left\{\lambda_{1}+k_{-}, \lambda_{1}+k_{+}\right\} .
\end{aligned}
$$

Then we have the following three assertions (see Figures 1.5 and 1.6):

(i) If $\lambda_{1}<\lambda<\min \left\{c_{1}, \lambda_{2}\right\}$, the semilinear problem (1.2) has two nontrivial solutions.

(ii) If $c_{1}<\min \left\{c_{2}, \lambda_{2}\right\}$ and $c_{1}<\lambda<\min \left\{c_{2}, \lambda_{2}\right\}$, the semilinear problem (1.2) has one nontrivial solution.

(iii) If $c_{2} \leq \lambda_{2}$ and $c_{2} \leq \lambda \leq \lambda_{2}$, the semilinear problem (1.2) has no nontrivial solutions.

(II) The bounded nonlinearity case: The second theorem asserts that if the function $f(t)$ is bounded, then the set of nontrivial solutions has properties considerably different from Theorem 1.2. More precisely, we have the following generalization of Szulkin [52, Theorem 5.1] to the degenerate case:

Theorem 1.3 Assume that hypotheses (H.1) and (H.2) are satisfied. Let $f(t)$ be a bounded, $C^{1}$ function on $\mathbf{R}$ such that

$$
f(0)=f^{\prime}(0)=0 .
$$

Moreover, we assume that $f^{\prime}(t)$ is decreasing for $t<0$ and increasing for $t>0$, near the origin $t=0$, and further that

$$
\sup _{|t|<\eta}\left|f^{\prime}(t)\right|<\lambda_{1}-\delta \quad \text { for some } \delta>0 \text { and } \eta>0 .
$$

Then the semilinear problem (1.2) has at least four nontrivial solutions $u_{1}, u_{2}, u_{3}$ and $u_{4}$ for each $\lambda \in\left(\lambda_{1}, \lambda_{1}+\varepsilon\right)$ provided that $\varepsilon>0$ is sufficiently small (see Figure 1.7).

For Theorem 1.3, we give a simple example of the function $f(t)$ :

Example 1.3 We let

$$
f(t):= \begin{cases}-\frac{1}{2 t}+\frac{2}{3} & \text { for } t>1 \\ \frac{t^{3}}{6} & \text { for }-1 \leq t \leq 1 \\ -\frac{1}{2 t}-\frac{2}{3} & \text { for } t<-1\end{cases}
$$

We may take $\eta:=\sqrt{\lambda_{1}}$ and $\delta:=\lambda_{1} / 4$. 


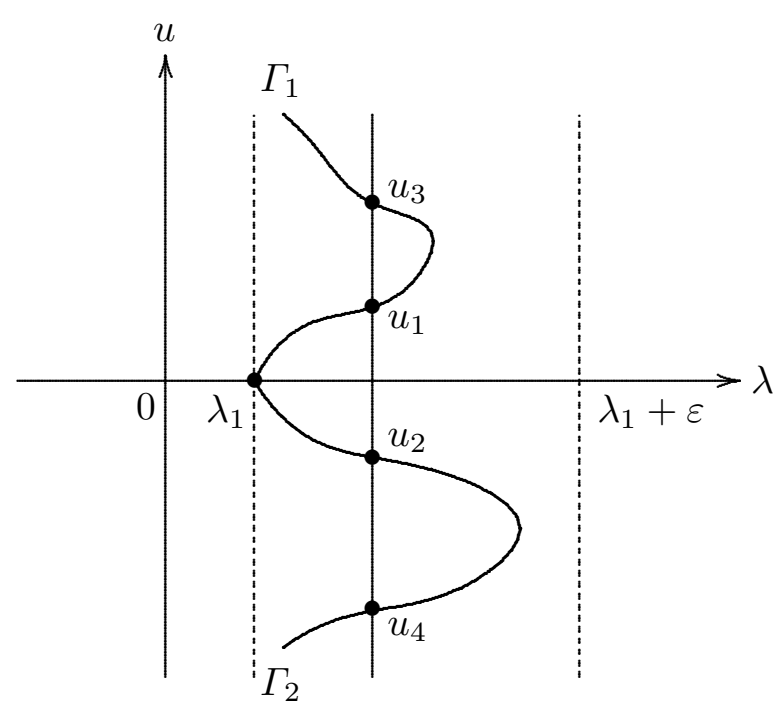

Fig. 1.7 The semilinear problem (1.2) has at least four nontrivial solutions $u_{1}, u_{2}, u_{3}$ and $u_{4}$

The third theorem is a generalization of Szulkin [52, Theorem 5.2] to the degenerate case, which asserts that the nontrivial solution branches turn back towards the first eigenvalue $\lambda_{1}$ :

Theorem 1.4 Assume that hypotheses (H.1) and (H.2) are satisfied and let $\lambda_{1}, \lambda_{2}$ be the first and second eigenvalues of $\mathfrak{A}$, respectively. Let $f(t)$ be a bounded, $C^{1}$ function on $\mathbf{R}$ such that $f(0)=0$ and $f^{\prime}(0)=0$. Moreover, we assume that there exists a constant $k>0$ such that

$$
0 \leq f^{\prime}(t) \leq k<\min \left\{\lambda_{1}, \lambda_{2}-\lambda_{1}\right\} \quad \text { for all } t \in \mathbf{R} .
$$

Then the set of nontrivial solutions of the semilinear problem (1.2), bifurcating at $\left(\lambda_{1}, 0\right)$, consists of two $C^{1}$ branches $\Gamma_{1}$ and $\Gamma_{2}$. The branches $\Gamma_{1}$ and $\Gamma_{2}$ may be parametrized respectively by $s$ as follows (see Figure 1.8):

$$
\begin{aligned}
& \Gamma_{1}=\left\{\left(\lambda^{(1)}(s), u_{1}(s)\right) \in \mathbf{R} \times C(\bar{D}): 0 \leq s<\infty\right\}, \\
& \Gamma_{2}=\left\{\left(\lambda^{(2)}(s), u_{2}(s)\right) \in \mathbf{R} \times C(\bar{D}): 0 \leq s<\infty\right\} .
\end{aligned}
$$

Moreover, $\left(\lambda^{(i)}(0), u_{i}(0)\right)=\left(\lambda_{1}, 0\right)$ and $\lambda^{(i)}(s) \rightarrow \lambda_{1}$ as $s \rightarrow \infty$, for $i=1$ and 2 .

Remark 1.4 Condition (1.5) implies that the distance between $\lambda_{1}$ and $\lambda_{2}$ determines the rate of the linear growth of the nonlinearity.

For Theorem 1.4, we give a simple example of the function $f(t)$ :

Example 1.4 If $k$ is a constant such that

$$
0<k<\min \left\{\lambda_{1}, \lambda_{2}-\lambda_{1}\right\}
$$




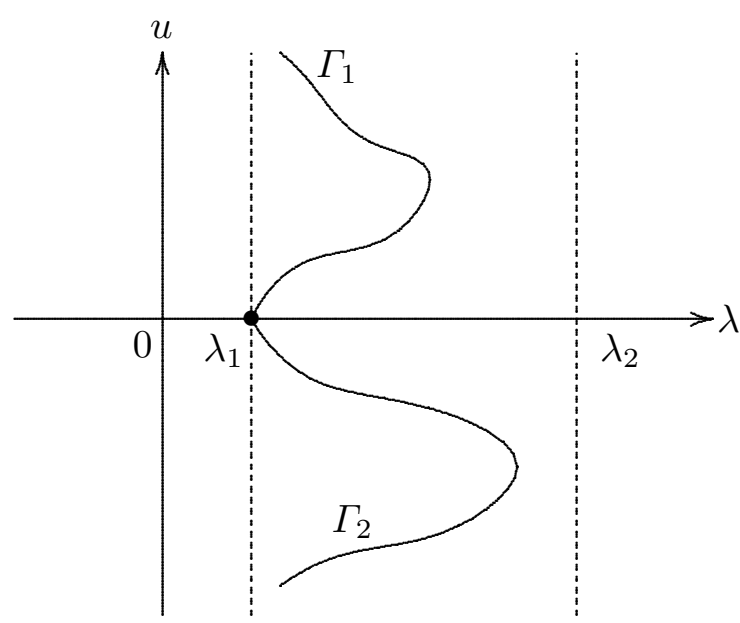

Fig. 1.8 The bifurcation curves $\Gamma_{1}$ and $\Gamma_{2}$ "turn back" towards the first eigenvalue $\lambda_{1}$

then we let

$$
f(t):= \begin{cases}k\left(-\frac{1}{2 t}+\frac{2}{3}\right) & \text { for } t>1 \\ \frac{k}{6} t^{3} & \text { for }-1 \leq t \leq 1, \\ k\left(-\frac{1}{2 t}-\frac{2}{3}\right) & \text { for } t<-1 .\end{cases}
$$

\subsection{Summary of the Contents}

The rest of this paper is organized as follows.

In Section 2 we study the linear boundary value problem (1.1) in the framework of $L^{2}$ spaces. Our class of semilinear second-order, elliptic boundary value problems satisfies the maximum principle. Roughly speaking, this additional information means that the operators associated with the boundary value problem (1.1) are compatible with the natural ordering of the underlying function spaces. Consequently, we are led to the study of nonlinear operator equations in the framework of ordered Banach spaces. The material in this section is given for completeness, to minimize the necessity of consulting many references.

By using the theory of ordered Banach spaces, we prove Theorem 1.1. More precisely, we prove Theorem 2.1, which plays an essential role in the proof of Theorem 1.2 in Section 4. On the way, we prove an existence and uniqueness theorem for problem (1.1) in the framework of $L^{p}$ Sobolev spaces and Hölder spaces (Theorems 2.2 and 2.3). Our approach is based on various maximum principles for second-order, elliptic differential operators with discontinuous coefficients such as the weak and strong maximum principles (Theorems A.1 and A.2) and the Hopf boundary point lemma (Lemma A.1) in the framework of $L^{p}$ Sobolev spaces.

Furthermore, the maximum principle tells us that the resolvent $K$ of the linear boundary value problem (1.1) is a positive operator in the framework of ordered Banach spaces. In order to obtain an abstract formulation of this fact, we introduce an ordered Banach subspace $C_{e}(\bar{D})$ of $C(\bar{D})$ (see formulas (2.4) and (2.5)), which combines the good properties of the resolvent $K$ with the good properties 
of the natural ordering of $C(\bar{D})$ (Proposition 2.2). The essential step in the proof is Proposition 2.2 where the compactness and strong positivity of the resolvent $K: C(\bar{D}) \rightarrow C_{e}(\bar{D})$ are proved. Theorem 2.6 (or rather Theorem 2.1) is an immediate consequence of the famous Krel̆n-Rutman theorem for strongly positive, compact linear operators (Theorem 2.5).

Section 3 is devoted to elements of topological methods in nonlinear analysis for the study of the semilinear elliptic boundary value problem (1.2). By using the bifurcation theory from a simple eigenvalue due to Crandall-Rabinowitz [26] and Dancer [27] (Theorems 3.3 and 3.4), we discuss the changes that occur in the structure of the solutions of the operator equation $F(\lambda, u)=0$ as $\lambda$ varies near the first eigenvalue $\lambda_{1}$ of the linear problem (1.1).

The Leray-Schauder degree is an important topological tool introduced by Leray-Schauder [39] in the study of nonlinear partial differential equations. The nontriviality of the degree guarantees the existence of a fixed point of the compact mapping in the domain (Theorem 3.6). It should be emphasized that the more precisely we know the degree the sharper we can estimate the number of fixed points. This opens a door to the study of multiple solutions in nonlinear analysis. The presentation here is taken from Brown [22], Chang [23] and Nirenberg [43].

In Section 4 we study the semilinear elliptic boundary value problem (1.2) and prove Theorem 1.2. The semilinear problem (1.2) is reduced to the study of a nonlinear operator equation for the resolvent $K$ of problem (1.1)

$$
\lambda K u-K(f(u))=u \quad \text { in } C(\bar{D}) .
$$

This nonlinear operator equation (1.6) is solved by using the theory of positive mappings in the ordered Banach space $C_{e}(\bar{D})$ (cf. [5], [27]), just as in Szulkin [52]. The idea of the proof of Theorem 1.2 may be stated as follows: By using Lemma 4.1, Lemma 4.5 and the implicit function theorem (Theorem 3.1), we can prove that the nonlinear operator equation (4.1) has a unique positive solution $u_{+}(\lambda)$ for all $\lambda_{1}<\lambda<\lambda_{1}+k_{+}$, and that the branch $\Gamma_{+}$of positive solutions emanating from $\left(\lambda_{1}, 0\right)$ is a $C^{1}$ curve given by the formula

$$
\Gamma_{+}=\left\{(\lambda, u) \in \mathbf{R} \times C(\bar{D}): u=u_{+}(\lambda), \lambda_{1} \leq \lambda<\lambda_{1}+k_{+}\right\} .
$$

The other branch $\Gamma_{-}$is obtained in a similar way. Furthermore, it follows from an application of Lemma 4.5 that no other positive or negative solutions exist for $\lambda>\lambda_{1}$, and also $\left\|u_{+}(\lambda)\right\| \rightarrow \infty$ as $\lambda \rightarrow \lambda_{1}+k_{+}$and $\left\|u_{-}(\lambda)\right\| \rightarrow \infty$ as $\lambda \rightarrow \lambda_{1}+k_{-}$. Finally, Lemma 4.6 tells us that there are no nontrivial solutions at $\lambda=\lambda_{1}$.

The proof of Theorems 1.3 and 1.4 is based on the Lyapunov-Schmidt procedure ([40], [50]) in Sections 6 and 7, which reduces an infinite-dimensional problem to a finite-dimensional system. In Section 5 we apply the Lyapunov-Schmidt procedure to the nonlinear operator equation (4.1) in the Banach space $C(\bar{D})$ (Theorem $5.1)$. This section is the heart of the subject, which is based on the previous works [57] and [58].

In Section 6 we prove Theorem 1.3 in the case where the nonlinear term $f(t)$ is bounded (Theorem 6.1). Our proof is based on an intermediate value argument, just as in the proof of Szulkin [52, Theorem 5.1] (see also Landesman-Lazer [38]). In the proof we make use of Amann-Ambrosetti-Mancini [7, Lemma 1.2] (Lemma 6.1). 
Section 7 is devoted to the proof of Theorem 1.4 in the case where the derivative $f^{\prime}(t)$ is bounded on $\mathbf{R}$ (Theorem 7.1). The proof of Theorem 1.4 is carried out by using the global theory of positive mappings due to Dancer [27] (Theorem 3.4), just as in the proof of Szulkin [52, Theorem 5.2].

In Section 8 we calculate the Leray-Schauder index of isolated critical points in the framework of the Lyapunov-Schmidt procedure as in Section 5 (Theorem 8.1). Finally, we apply Theorem 8.1 (or Corollary 8.1) to the semilinear elliptic boundary value problem (1.2) with $\lambda:=\lambda_{1}$, that is, the resonance case:

$$
\begin{cases}A u-\lambda_{1} u+f(u)=0 & \text { in } D, \\ B u=0 & \text { on } \partial D .\end{cases}
$$

We give sufficient conditions in order that this semilinear elliptic boundary value problem at resonance has at least two nontrivial solutions (Theorem 8.2).

In the final Section 9, for the semilinear elliptic boundary value problem (1.2) we give an overview of the classical Schauder theory in Szulkin [52] versus the $L^{p}$ theory of pseudo-differential operators in the present paper (Table 9.1).

The weak and strong maximum principles (Theorems A.1 and A.2) and the Hopf boundary point lemma (Lemma A.1) are summarized in the Appendix.

\section{Linear elliptic boundary value problems}

In this section we consider the linearized boundary value problem (1.1) in the framework of $L^{2}$ spaces, and prove Theorem 1.1. More precisely, we prove the following theorem (see [53, Theorem 7.3], [55, Theorem 1]):

Theorem 2.1 Assume that hypotheses (H.1) and (H.2) are satisfied. Then we have the following two assertions:

(i) The first eigenvalue $\lambda_{1}$ of $\mathfrak{A}$ is positive and algebraically simple, and its associated eigenfunction $\varphi_{1}$ is positive everywhere in $D$.

(ii) No other eigenvalues have positive eigenfunctions.

We remark that Taira [55, Theorem 1] proved Theorem 2.1 by making use of the theory of Feller semigroups in functional analysis. In this paper we shall give a simple and direct proof of Theorem 2.1 by making use of the theory of positive mappings in ordered Banach spaces (cf. [5], [22], [29], [36]).

\subsection{Function spaces}

Let $D$ be an open set in Euclidean space $\mathbf{R}^{N}$.

(I) First, we let

$$
C(D)=\text { the space of continuous functions in } D \text {. }
$$

If $k$ is a positive integer, we let

$$
C^{k}(D)=\text { the space of functions of class } C^{k} \text { in } D .
$$


Furthermore, we let

$$
\begin{aligned}
C(\bar{D})= & \text { the space of functions in } C(D) \text { having continuous extensions } \\
& \text { to the closure } \bar{D} \text { of } D .
\end{aligned}
$$

If $k$ is a positive integer, we let

$$
\begin{aligned}
C^{k}(\bar{D})= & \text { the space of functions in } C^{k}(D) \text { all of whose derivatives } \\
& \text { of order } \leq k \text { have continuous extensions to } \bar{D} .
\end{aligned}
$$

Let $0<\theta<1$. A function $u$ defined on $D$ is said to be uniformly Hölder continuous with exponent $\theta$ in $D$ if the quantity

$$
[u]_{\theta ; D}=\sup _{\substack{x, y \in D \\ x \neq y}} \frac{|u(x)-u(y)|}{|x-y|^{\alpha}}
$$

is finite. We say that $u$ is locally Hölder continuous with exponent $\theta$ in $D$ if it is uniformly Hölder continuous with exponent $\theta$ on compact subsets of $D$.

If $0<\theta<1$, we define the Hölder space $C^{\theta}(D)$ as follows:

$$
\begin{aligned}
C^{\theta}(D)= & \text { the space of functions in } C(D) \text { which are locally Hölder } \\
& \text { continuous with exponent } \theta \text { in } D .
\end{aligned}
$$

If $k$ is a positive integer and $0<\theta<1$, we define the Hölder space $C^{k+\theta}(D)$ as follows:

$$
\begin{aligned}
C^{k+\theta}(D)= & \text { the space of functions in } C^{k}(D) \text { all of whose } k \text {-th order } \\
& \text { derivatives are locally Hölder continuous with exponent } \theta \text { in } D .
\end{aligned}
$$

Furthermore, we let

$$
\begin{aligned}
C^{\theta}(\bar{D})= & \text { the space of functions in } C(\bar{D}) \text { which are Hölder } \\
& \text { continuous with exponent } \theta \text { on } \bar{D}
\end{aligned}
$$

and

$$
\begin{aligned}
C^{k+\theta}(\bar{D})= & \text { the space of functions in } C^{k}(\bar{D}) \text { all of whose } k \text {-th order } \\
& \text { derivatives are Hölder continuous with exponent } \theta \text { on } \bar{D} .
\end{aligned}
$$

Let $k$ be a non-negative integer and $0<\theta<1$. We introduce various seminorms on the spaces $C^{k}(D)$ and $C^{k+\theta}(D)$ as follows:

$$
\begin{aligned}
& {[u]_{k, 0 ; D}=\left|D^{k} u\right|_{0 ; D}=\sup _{x \in D|\beta|=k} \sup _{|\beta|}\left|D^{\beta} u(x)\right|,} \\
& {[u]_{k, \theta ; D}=\left[D^{k} u\right]_{\theta ; D}=\sup _{|\beta|=k}\left[D^{\beta} u\right]_{\theta ; D} .}
\end{aligned}
$$

We can define the associated norms on the spaces $C^{k}(\bar{D})$ and $C^{k+\theta}(\bar{D})$ as follows:

$$
\|u\|_{C^{k}(\bar{D})}=|u|_{k ; \bar{D}}=\sum_{j=0}^{k}\left|D^{j} u\right|_{0 ; \bar{D}}
$$




$$
\|u\|_{C^{k+\theta}(\bar{D})}=|u|_{k, \theta ; \bar{D}}=|u|_{k ; \bar{D}}+\left[D^{k} u\right]_{\theta ; \bar{D}} .
$$

The Hölder spaces $C^{k+\theta}(\partial D)$ on the smooth boundary $\partial D$ are defined to be locally the Hölder spaces $C^{k+\theta}\left(\mathbf{R}^{N-1}\right)$, upon using local coordinate systems flattening out $\partial D$, together with a partition of unity.

(II) Secondly, if $1 \leq p<\infty$, we let

$$
\begin{aligned}
L^{p}(D)= & \text { the space of (equivalence classes of) Lebesgue measurable } \\
& \text { functions } u \text { on } D \text { such that }|u|^{p} \text { is integrable on } D .
\end{aligned}
$$

The space $L^{p}(D)$ is a Banach space with the norm

$$
\|u\|_{L^{p}(D)}=\left(\int_{D}|u(x)|^{p} d x\right)^{1 / p}
$$

If $k$ is a positive integer and $1<p<\infty$, we define a Sobolev space

$$
\begin{aligned}
W^{k, p}(D)= & \text { the space of functions } u \in L^{p}(D) \text { whose derivatives } \\
& D^{\alpha} u,|\alpha| \leq k, \text { in the sense of distributions are in } L^{p}(D) .
\end{aligned}
$$

The space $W^{k, p}(D)$ is a Banach space with the norm

$$
\|u\|_{W^{k, p}(D)}=\left(\sum_{|\alpha| \leq k} \int_{D}\left|D^{\alpha} u(x)\right|^{p} d x\right)^{1 / p} .
$$

Furthermore, if the boundary $\partial D$ is smooth, we let

$$
\begin{aligned}
B^{k-1 / p, p}(\partial D)= & \text { the space of the boundary values } \varphi=\left.u\right|_{\partial D} \\
& \text { of functions } u \in W^{k, p}(D) .
\end{aligned}
$$

In the space $B^{k-1 / p, p}(\partial D)$, we introduce a norm

$$
|\varphi|_{B^{k-1 / p, p}(\partial D)}=\inf \left\{\|u\|_{W^{k, p}(D)}: \varphi=\left.u\right|_{\partial D} \text { for } u \in W^{k, p}(D)\right\} .
$$

The space $B^{k-1 / p, p}(\partial D)$ is a Banach space with respect to the norm $|\cdot|_{B^{k-1 / p, p}(\partial D)}$; more precisely, it is a Besov space (cf. [1], [19], [71]).

2.2 Existence and uniqueness theorem for the linear problem (1.1)

In this subsection we study the linear elliptic boundary value problem (1.1) in the framework of Hölder spaces. To do so, we introduce a subspace of the Hölder space $C^{1+\theta}(\partial D)$ for $0<\theta<1$, which is associated with the degenerate boundary condition

$$
B u=a\left(x^{\prime}\right) \frac{\partial u}{\partial \boldsymbol{\nu}}+b\left(x^{\prime}\right) u
$$

under hypotheses (H.1) and (H.2) in the following way: We let

$$
C_{\star}^{1+\theta}(\partial D):=a\left(x^{\prime}\right) C^{1+\theta}(\partial D)+b\left(x^{\prime}\right) C^{2+\theta}(\partial D)
$$




$$
=\left\{\varphi=a\left(x^{\prime}\right) \varphi_{1}+b\left(x^{\prime}\right) \varphi_{2}: \varphi_{1} \in C^{1+\theta}(\partial D), \varphi_{2} \in C^{2+\theta}(\partial D)\right\}
$$

and define a norm

$$
|\varphi|_{C_{\star}^{1+\theta}(\partial D)}=\inf \left\{\left|\varphi_{1}\right|_{C^{1+\theta}(\partial D)}+\left|\varphi_{2}\right|_{C^{2+\theta}(\partial D)}: \varphi=a\left(x^{\prime}\right) \varphi_{1}+b\left(x^{\prime}\right) \varphi_{2}\right\} .
$$

Then it is easy to verify that the space $C_{\star}^{1+\theta}(\partial D)$ is a Banach space with respect to the norm $|\cdot|_{C_{\star}^{1+\theta}(\partial D)}$. We remark that the space $C_{\star}^{1+\theta}(\partial D)$ is an "interpolation space" between the spaces $C^{2+\theta}(\partial D)$ and $C^{1+\theta}(\partial D)$. More precisely, we have the assertions

$$
\begin{aligned}
& C_{\star}^{1+\theta}(\partial D)=a\left(x^{\prime}\right) C^{1+\theta}(\partial D)+b\left(x^{\prime}\right) C^{2+\theta}(\partial D) \\
= & \begin{cases}C^{2+\theta}(\partial D) & \text { if } a\left(x^{\prime}\right) \equiv 0 \text { on } \partial D \text { (the Dirichlet case), } \\
C^{1+\theta}(\partial D) & \text { if } b\left(x^{\prime}\right) \equiv 0 \text { on } \partial D \text { (the Neumann case), } \\
C^{1+\theta}(\partial D) & \text { if } a\left(x^{\prime}\right)>0 \text { on } \partial D \text { (the regular Robin case), }\end{cases}
\end{aligned}
$$

and, for general $a\left(x^{\prime}\right)$, the continuous injections

$$
C^{2+\theta}(\partial D) \subset C_{\star}^{1+\theta}(\partial D) \subset C^{1+\theta}(\partial D) .
$$

The purpose of this subsection is to prove the following theorem (see [54, Theorem 1.1]):

Theorem 2.2 If hypotheses (H.1) and (H.2) are satisfied, then the mapping

$$
(A, B): C^{2+\theta}(\bar{D}) \longrightarrow C^{\theta}(\bar{D}) \oplus C_{\star}^{1+\theta}(\partial D)
$$

is an algebraic and topological isomorphism for all $0<\theta<1$.

Proof The proof is divided into four steps.

Step 1: Let $g$ be an arbitrary element of $C^{\theta}(\bar{D})$, and $\varphi$ an arbitrary element of $C_{\star}^{1+\theta}(\partial D)$ such that

$$
\varphi=a\left(x^{\prime}\right) \varphi_{1}+b\left(x^{\prime}\right) \varphi_{2}, \quad \varphi_{1} \in C^{1+\theta}(\partial D), \varphi_{2} \in C^{2+\theta}(\partial D) .
$$

First, we show that the boundary value problem

$$
\begin{cases}A u=g & \text { in } D, \\ B u=\varphi & \text { on } \partial D\end{cases}
$$

can be reduced to the study of an operator on the boundary. To do this, we consider the (purely) Neumann problem

$$
\begin{cases}A v=g & \text { in } D \\ \frac{\partial v}{\partial \boldsymbol{\nu}}=\varphi_{1} & \text { on } \partial D .\end{cases}
$$

By [30, Theorem 6.31], we can find a unique solution $v$ in the space $C^{2+\theta}(\bar{D})$ of the Neumann problem $(\mathrm{N})$. Then it is easy to see that a function $u$ in $C^{2+\theta}(\bar{D})$ 
is a solution of the boundary value problem (2.1) if and only if the function $w=$ $u-v \in C^{2+\theta}(\bar{D})$ is a solution of the problem

$$
\begin{cases}A w=0 & \text { in } D, \\ B w=\varphi-B v & \text { on } \partial D .\end{cases}
$$

Here we remark that

$$
B v=a\left(x^{\prime}\right) \frac{\partial v}{\partial \nu}+b\left(x^{\prime}\right) v=a\left(x^{\prime}\right) \varphi_{1}+b\left(x^{\prime}\right) v
$$

so that

$$
B w=\varphi-B v=b\left(x^{\prime}\right)\left(\varphi_{2}-v\right) \in C^{2+\theta}(\partial D) .
$$

However, we know that every solution $w \in C^{2+\theta}(\bar{D})$ of the homogeneous equation: $A w=0$ in $D$ can be expressed as follows (cf. [31, Chapter XX], [49, Chapter 3]):

$$
w=\mathcal{P} \psi, \quad \psi \in C^{2+\theta}(\partial D) .
$$

Here the operator

$$
\mathcal{P}: C^{2+\theta}(\partial D) \longrightarrow C^{2+\theta}(\bar{D})
$$

is the Poisson kernel, that is, the function $w=\mathcal{P} \psi$ is the unique solution of the Dirichlet problem

$$
\begin{cases}A w=0 & \text { in } D, \\ w=\psi & \text { on } \partial D .\end{cases}
$$

Thus we have the following proposition:

Proposition 2.1 For given functions $g \in C^{\theta}(\bar{D})$ and $\varphi=a\left(x^{\prime}\right) \varphi_{1}+b\left(x^{\prime}\right) \varphi_{2} \in$ $C_{\star}^{1+\theta}(\partial D)$, there exists a solution $u \in C^{2+\theta}(\bar{D})$ of the boundary value problem (2.1) if and only if there exists a solution $\psi \in C^{2+\theta}(\partial D)$ of the equation

$$
T \psi:=B \mathcal{P} \psi=b\left(x^{\prime}\right)\left(\varphi_{2}-v\right) \text { on } \partial D .
$$

Furthermore, the solutions $u$ and $\psi$ are related as follows:

$$
u=v+\mathcal{P} \psi,
$$

where $v \in C^{2+\theta}(\bar{D})$ is the unique solution of the Neumann problem (N).

Remark 2.1 The equation (2.2) is a modern version of the classical Fredholm integral equation.

Step 2: We study the operator $T$ in question. It is known (cf. [31, Chapter $\mathrm{XX}]$, [49, Chapter 3]) that the operator

$$
T \psi=B \mathcal{P} \psi=a\left(x^{\prime}\right) \frac{\partial}{\partial \boldsymbol{\nu}}(\mathcal{P} \psi)+b\left(x^{\prime}\right) \psi
$$

is a first-order, pseudo-differential operator on the boundary $\partial D$.

The next lemma is an essential step in the proof of Theorem 2.2 (see [66, Lemma 6.1]):

Lemma 2.1 If hypotheses (H.1) and (H.2) are satisfied, then there exists a parametrix $E$ in the Hörmander class $L_{1,1 / 2}^{0}(\partial D)$ for $T$ that maps $C^{k+\theta}(\partial D)$ continuously into itself for all non-negative integers $k$. 
Proof By making use of [31, Theorem 22.1.3] just as in the proof of [65, Lemma 7.3], we can construct a parametrix $E \in L_{1,1 / 2}^{0}(\partial D)$ for $T$ :

$$
E T \equiv T E \equiv I \quad \bmod L^{-\infty}(\partial D) .
$$

The boundedness of $E: C^{k+\theta}(\partial D) \rightarrow C^{k+\theta}(\partial D)$ follows from an application of a Besov-space boundedness theorem due to Bourdaud [21, Theorem 1] (see [65, Theorem 5.15] with $m:=0, \delta:=1 / 2$ and $p:=\infty$ ), since we have the assertion (see [71, Chapter 1])

$$
C^{k+\theta}(\partial D)=B_{\infty, \infty}^{k+\theta}(\partial D) .
$$

The proof of Lemma 2.1 is complete.

Step 3: We consider the boundary value problem (2.1) in the framework of Sobolev spaces of $L^{p}$ style, and prove an $L^{p}$ version of Theorem 2.2 (see [69, Part III]).

We introduce a subspace of $B^{1-1 / p, p}(\partial D)$ which is an $L^{p}$ version of $C_{\star}^{1+\theta}(\partial D)$ under hypotheses (H.1) and (H.2). We let

$$
\begin{aligned}
& B_{\star}^{1-1 / p, p}(\partial D) \\
= & \left\{\varphi=a\left(x^{\prime}\right) \varphi_{1}+b\left(x^{\prime}\right) \varphi_{2}: \varphi_{1} \in B^{1-1 / p, p}(\partial D), \varphi_{2} \in B^{2-1 / p, p}(\partial D)\right\},
\end{aligned}
$$

and define a norm

$$
\begin{aligned}
& |\varphi|_{B_{\star}^{1-1 / p, p}(\partial D)} \\
= & \inf \left\{\left|\varphi_{1}\right|_{B^{1-1 / p, p}(\partial D)}+\left|\varphi_{2}\right|_{B^{2-1 / p, p}(\partial D)}: \varphi=a\left(x^{\prime}\right) \varphi_{1}+b\left(x^{\prime}\right) \varphi_{2}\right\} .
\end{aligned}
$$

It is easy to verify that the space $B_{\star}^{1-1 / p, p}(\partial D)$ is a Banach space with respect to the norm $|\cdot|_{B_{\star}^{1-1 / p, p}(\partial D)}$.

Then we can obtain the following $L^{p}$ version of Theorem 2.2 (see [65, Theorem $1.1])$ :

Theorem 2.3 If hypotheses (H.1) and (H.2) are satisfied, then the mapping

$$
(A, B): W^{2, p}(D) \longrightarrow L^{p}(D) \oplus B_{\star}^{1-1 / p, p}(\partial D)
$$

is an algebraic and topological isomorphism for all $1<p<\infty$.

Step 4: Now we remark that

$$
\left\{\begin{array}{l}
C^{\theta}(\bar{D}) \subset L^{p}(D), \\
C_{\star}^{1+\theta}(\partial D) \subset B_{\star}^{1-1 / p, p}(\partial D) .
\end{array}\right.
$$

Thus we find from Theorem 2.3 that the boundary value problem (2.1) has a unique solution $u \in W^{2, p}(D)$ for any $g \in C^{\theta}(\bar{D})$ and any $\varphi=a\left(x^{\prime}\right) \varphi_{1}+b\left(x^{\prime}\right) \varphi_{2} \in$ $C_{\star}^{1+\theta}(\partial D)$. Moreover, by virtue of Proposition 2.1 it follows that the solution $u$ can be written in the form

$$
u=v+\mathcal{P} \psi, \quad v \in C^{2+\theta}(\bar{D}), \psi \in B^{2-1 / p, p}(\partial D) .
$$


However, Lemma 2.1 tells us that

$$
\psi \in C^{2+\theta}(\partial D)
$$

since we have, by equation (2.2),

$$
\psi \equiv E(T \psi)=E\left(b\left(x^{\prime}\right)\left(\varphi_{2}-v\right)\right) \quad \bmod C^{\infty}(\partial D) .
$$

Therefore, we obtain that

$$
u=v+\mathcal{P} \psi \in C^{2+\theta}(\bar{D}) .
$$

The proof of Theorem 2.2 is complete.

2.3 Proof of Theorem 2.1 - Part $1-$

First, we let

$$
C_{B}^{2+\theta}(\bar{D})=\left\{u \in C^{2+\theta}(\bar{D}): B u=0 \text { on } \partial D\right\} .
$$

By Theorem 2.2, we can define a resolvent

$$
K: C^{\theta}(\bar{D}) \longrightarrow C_{B}^{2+\theta}(\bar{D})
$$

for the linear problem (1.1) as follows: For any $f \in C^{\theta}(\bar{D})$, the function $u=K f \in$ $C^{2+\theta}(\bar{D})$ is the unique solution of the problem

$$
\begin{cases}A u=f & \text { in } D, \\ B u=0 & \text { on } \partial D .\end{cases}
$$

Secondly, we let

$$
W_{B}^{2, p}(D)=\left\{u \in W^{2, p}(D): B u=0 \text { on } \partial D\right\} .
$$

By Theorem 2.3, we can define a resolvent

$$
K: L^{p}(D) \longrightarrow W_{B}^{2, p}(D)
$$

for the linear elliptic boundary value problem (1.1) as follows: For any $f \in L^{p}(D)$, the function $u=K f \in W^{2, p}(D)$ is the unique solution of the problem

$$
\begin{cases}A u=f & \text { in } D, \\ B u=0 & \text { on } \partial D .\end{cases}
$$

Furthermore, by the Ascoli-Arzelà theorem (see [30, Lemma 6.36]) it follows that the operator $K$, considered as

$$
K: C(\bar{D}) \longrightarrow C^{1}(\bar{D}),
$$

is compact if $N<p<\infty$. Indeed, by Sobolev's imbedding theorem (see [1, Theorem 4.12 , Part II]) it suffices to note that the space $W^{2, p}(D)$ is continuously imbedded 


$$
K: C(\bar{D}) \longrightarrow L^{p}(D) \stackrel{K}{\longrightarrow} W^{2, p}(D) \longrightarrow C^{2-N / p}(\bar{D}) \underset{\text { compactly }}{\hookrightarrow \hookrightarrow} C^{1}(\bar{D}) .
$$

Fig. 2.1 The mapping properties of the resolvent $K$

into $C^{2-N / p}(\bar{D})$ with $2-N / p>1$, for all $N<p<\infty$. The situation can be visualized as in Figure 2.1.

Then it follows from an application of regularity theorem for the linear elliptic boundary value problem (1.1) ([65, Theorem 7.1]) that $u \in L^{p}(D)$ for $1<p<\infty$ is a solution of the eigenvalue problem

$$
\begin{cases}A u=\lambda u & \text { in } D, \\ B u=0 & \text { on } \partial D\end{cases}
$$

if and only if it satisfies the operator equation

$$
u=\lambda K u \quad \text { in } C(\bar{D}) .
$$

2.4 Theory of positive mappings in ordered Banach spaces

We shall make use of the theory of positive operators in ordered Banach spaces to study positive solutions of equation (2.3) (cf. [5], [22, Chapter 18], [29, Chapter $6],[36])$.

Let $X$ be a non-empty set. An ordering $\leq$ in $X$ is a relation in $X$ that is reflexive, antisymmetric and transitive:

(a) $x \leq x$ for all $x \in X$ (reflexivity).

(b) If $x \leq y$ and $y \leq x$, then we have $x=y$ for all $x$ and $y \in X$ (antisymmetry).

(c) If $x \leq y$ and $y \leq z$, then we have $x \leq z$ for all $x, y$ and $z \in X$ (transitivity).

A non-empty set together with an ordering is called an ordered set.

Let $V$ be a real vector space. An ordering $\leq$ in $V$ is said to be linear if the following two conditions are satisfied:

(i) If $x, y \in V$ and $x \leq y$, then we have $x+z \leq y+z$ for all $z \in V$.

(ii) If $x, y \in V$ and $x \leq y$, then we have $\alpha x \leq \alpha y$ for all $\alpha \geq 0$.

A real vector space together with a linear ordering is called an ordered vector space.

If $x, y \in V$ and $x \leq y$, then the set $[x, y]=\{z \in X: x \leq z \leq y\}$ is called an order interval.

Let $V$ be an ordered vector space. If we let

$$
Q=\{x \in V: x \geq 0\},
$$

then it is easy to verify that the set $Q$ has the following two conditions:

(iii) If $x, y \in Q$, then $\alpha x+\beta y \in Q$ for all $\alpha, \beta \geq 0$.

(iv) If $x \neq 0$, then at least one of $x$ and $-x$ does not belong to $Q$, or equivalently, $Q \cap(-Q)=\{0\}$. 
The set $Q$ is called the positive cone of the ordering $\leq$.

Let $E$ be a Banach space $E$ with a linear ordering $\leq$. The Banach space $E$ is called an ordered Banach space if the positive cone

$$
Q=\{x \in E: x \geq 0\}
$$

is closed in $E$. We say that $Q$ is generating if, for each $x \in E$ there exist vectors $u, v \in Q$ such that $x=u-v$. It is to be expected that the topology and the ordering of an ordered Banach space are closely related if the norm is monotone: If $0 \leq u \leq v$, then $\|u\| \leq\|v\|$.

For $x, y \in E$, we write

$$
\begin{array}{ll}
x \geq y & \text { if } x-y \in Q, \\
x>y & \text { if } x-y \in Q \backslash\{0\} .
\end{array}
$$

If the interior $\operatorname{Int}(Q)$ is non-empty, then we write

$$
x \gg y \quad \text { if } x-y \in \operatorname{Int}(Q) .
$$

Here we give two simple but important examples of ordered Banach spaces:

Example 2.1 Let $E=\mathbf{R}^{N}$, and let

$$
\mathbf{R}^{N,+}=\left\{x=\left(x_{1}, x_{2}, \ldots, x_{N}\right) \in \mathbf{R}^{N}: x_{i} \geq 0 \text { for all } 1 \leq i \leq N\right\} .
$$

For any $u, v \in \mathbf{R}^{N}$, we write $u \leq v$ if $v-u \in \mathbf{R}^{N,+}$. Then it is easy to see that $\left(\mathbf{R}^{N}, \mathbf{R}^{N,+}, \leq\right)$ is an ordered Banach space and that the norm is monotone. Moreover, the positive cone $\mathbf{R}^{N,+}$ is generating. We remark that

$$
\operatorname{Int}\left(\mathbf{R}^{N,+}\right)=\left\{x=\left(x_{1}, x_{2}, \ldots, x_{N}\right) \in \mathbf{R}^{N}: x_{i}>0 \text { for all } 1 \leq i \leq N\right\} .
$$

Example 2.2 Let $E=C(\bar{D})$ be the set of real-valued, continuous functions on the closure $\bar{D}$, and let

$$
P=\{u \in C(\bar{D}): u(x) \geq 0 \text { on } \bar{D}\} .
$$

For any $u, v \in C(\bar{D})$, we write $u \leq v$ if $v-u \in P$. Then it is easy to see that $(C(\bar{D}), P)$ is an ordered Banach space and that the norm is monotone. Moreover, the positive cone $P$ is generating. We remark that

$$
\operatorname{Int}(P)=\{u \in C(\bar{D}): u(x)>0 \text { on } \bar{D}\} .
$$

A linear operator $L: E \rightarrow E$ is said to be positive if $L$ maps $P$ into itself:

$$
L: P \longrightarrow P \text {. }
$$

A linear operator $L: E \rightarrow E$ is said to be strictly positive if $L$ maps $P \backslash\{0\}$ into itself:

$$
L: P \backslash\{0\} \longrightarrow P \backslash\{0\} .
$$

A linear operator $L: E \rightarrow E$ is said to be strongly positive if $L x$ is an interior point of $P$ for every $x \in P \backslash\{0\}$ :

$$
L: P \backslash\{0\} \longrightarrow \operatorname{Int}(P) .
$$


A linear operator $L: E \rightarrow E$ is said to be compact if it is continuous (bounded) and maps bounded sets into relatively compact sets.

The next famous Krel̆n-Rutman theorem for strongly positive, compact linear operators will play a fundamental role in the sequel (see [36, Chapter 2], [23, Theorem 3.6.12], [29, Chapter 6, Theorem 6.3.35]):

Theorem 2.4 (Kreŭn-Rutman) Let $E$ be an ordered Banach space with total positive cone $P$. Assume that $T: E \rightarrow E$ is a compact linear operator such that its spectral radius

$$
r(T)=\lim _{k \rightarrow \infty} \sqrt[k]{\left\|T^{k}\right\|}
$$

is positive. Then $r(T)$ is an eigenvalue of $T$ with eigenvector in $P$ and an eigenvalue of the dual operator

$$
T^{*}: E^{*} \longrightarrow E^{*}
$$

with eigenvector in the dual wedge $P^{*}$ of $P$, respectively. Here

$$
P^{*}=\text { the space of all continuous, positive linear functionals on } E \text {. }
$$

Example 2.3 (Perron-Frobenius) Let $\left(\mathbf{R}^{N}, \mathbf{R}^{N,+}, \leq\right)$ be the ordered Banach space in Example 2.1. If $L=\left(a_{i j}\right)$ is a transition matrix of positive entries $a_{i j}$ of a Markov chain such that

$$
\begin{aligned}
& \sum_{j=1}^{N} a_{i j}=1 \quad \text { for each } 1 \leq i \leq N \\
& \sum_{k=1}^{N} a_{k j}=1 \quad \text { for each } 1 \leq j \leq N
\end{aligned}
$$

then it follows that $L: E \rightarrow E$ is a linear, compact and strongly positive operator. It is easy to see that

$$
\begin{aligned}
& r(L)=\lim _{n \rightarrow \infty} \sqrt[n]{\left\|L^{n}\right\|}=1, \\
& x_{0}=(1,1, \ldots, 1) \in \mathbf{R}^{N,+} .
\end{aligned}
$$

This is a special case of the classical Perron-Frobenius theorem.

Furthermore, we can obtain a sharp version of Theorem 2.4 in the case where the operator $T: E \rightarrow E$ is strongly positive ([29, Chapter 6, Theorem 6.3.35]):

Theorem 2.5 (Kreŭn-Rutman) Let $E$ be an ordered Banach space with positive cone $P$ having non-empty interior Int $(P)$. Assume that

$$
T: E \longrightarrow E
$$

is a strongly positive, compact linear operator. Then we have the following five assertions:

(i) The spectral radius $r(T)$ is positive.

(ii) $r(T)$ is an algebraically simple eigenvalue of $T$ having a positive eigenvector $x_{0} \in \operatorname{Int}(P): T x_{0}=r(T) x_{0}$. There exist no other eigenvalues with a positive eigenvector. 
(iii) $r(T)$ is an algebraically simple eigenvalue of the dual operator $T^{*}: E^{*} \rightarrow E^{*}$ having a strictly positive eigenvector $x_{0}^{*} \in P^{*} \backslash\{0\}$. Namely, $x_{0}^{*}$ satisfies the condition

$$
\left\langle x, x_{0}^{*}\right\rangle>0 \quad \text { for all } x \in P \backslash\{0\} .
$$

(iv) For every $y \in P \backslash\{0\}$, the equation

$$
\lambda x-T x=y
$$

has exactly one positive solution if $\lambda>r(T)$, and no positive solution for $\lambda \leq r(T)$. The equation

$$
r(T) x-T x=-y
$$

has no positive solution.

(v) For every continuous linear operator $S$ satisfying $S \geq T$, we have the assertion

$$
r(S) \geq r(T) .
$$

If $S-T$ is strongly positive, then we have the assertion

$$
r(S)>r(T) .
$$

2.5 The ordered Banach space $C_{e}(\bar{D})$

Now we introduce an ordered Banach space that is associated with the operator (see Figure 2.1)

$$
K: C(\bar{D}) \longrightarrow C^{1}(\bar{D}) .
$$

To do this, we need the following lemma (see [56, Lemma 3.7]):

Lemma 2.2 Assume that hypotheses (H.1) and (H.2) are satisfied. If $v \in C^{\theta}(\bar{D})$ with $0<\theta<1$ and if $v \in P \backslash\{0\}$, then the function $u=K v \in C^{2+\theta}(\bar{D})$ satisfies the following three conditions:

(a) $u\left(x^{\prime}\right)=0$ on $M=\left\{x^{\prime} \in \partial D: a\left(x^{\prime}\right)=0\right\}$.

(b) $u(x)>0$ on $\bar{D} \backslash M$.

(c) For the conormal derivative $\partial u / \partial \boldsymbol{\nu}$ of $u$, we have the inequality

$$
\frac{\partial u}{\partial \boldsymbol{\nu}}\left(x^{\prime}\right)<0 \quad \text { on } M
$$

In particular, the operator $K: C(\bar{D}) \rightarrow C(\bar{D})$ is strictly positive:

$$
v>0 \Longrightarrow K v>0
$$

Proof The proof of Lemma 2.2 is divided into three steps.

Step 1: First, since the function $u=K v \in C^{2+\theta}(\bar{D})$ satisfies the condition

$$
A u=v \geq 0 \quad \text { in } D,
$$

it follows from an application of the weak maximum principle (Theorem A.1) that the function $u$ may take its negative minimum only on the boundary $\partial D$.

However, we have the following claim: 
Claim 2.1 The function $u=K v$ does not take its negative minimum on the boundary $\partial D$. In other words, the function $u$ is non-negative on $\bar{D}$.

Proof Our proof is based on a reduction to absurdity. Assume, to the contrary, that there exists a point $x_{0}^{\prime} \in \partial D$ such that

$$
u\left(x_{0}^{\prime}\right)<0
$$

If $a\left(x_{0}^{\prime}\right)=0$, then we have, by condition (H.2),

$$
0=B u\left(x_{0}^{\prime}\right)=b\left(x_{0}^{\prime}\right) u\left(x_{0}^{\prime}\right)<0 .
$$

This is a contradiction.

If $a\left(x_{0}^{\prime}\right)>0$, then it follows that

$$
\left\{\begin{array}{l}
A u(x)=v(x) \geq 0 \quad \text { for all } x \in D \\
u\left(x_{0}^{\prime}\right)=\min _{\bar{D}} u<0 \\
u(x)>u\left(x_{0}^{\prime}\right) \quad \text { for all } x \in D
\end{array}\right.
$$

Thus it follows from an application of the Hopf boundary point lemma (Lemma A.1) that

$$
\frac{\partial u}{\partial \boldsymbol{\nu}}\left(x_{0}^{\prime}\right)<0 .
$$

However, by hypotheses (H.1) and (H.2) it follows that

$$
a\left(x^{\prime}\right)+b\left(x^{\prime}\right)>0 \quad \text { on } \partial D .
$$

Hence we have the assertion

$$
0=B u\left(x_{0}^{\prime}\right)=a\left(x_{0}^{\prime}\right) \frac{\partial u}{\partial \boldsymbol{\nu}}\left(x_{0}^{\prime}\right)+b\left(x_{0}^{\prime}\right) u\left(x_{0}^{\prime}\right) \leq a\left(x_{0}^{\prime}\right) \frac{\partial u}{\partial \boldsymbol{\nu}}\left(x_{0}^{\prime}\right)<0 .
$$

This is also a contradiction.

Step 2: Furthermore, we have the following claim:

Claim 2.2 The function $u=K v$ is strictly positive in $D$, that is,

$$
u(x)=K v(x)>0 \quad \text { in } D .
$$

Proof Our proof is based on a reduction to absurdity. Assume, to the contrary, that there exists a point $x_{0} \in D$ such that

$$
u\left(x_{0}\right)=0 .
$$

Then we obtain from the strong maximum principle (Theorem A.2) that

$$
u(x) \equiv 0 \quad \text { in } D,
$$

so that

$$
v=K u=0 \quad \text { in } D .
$$

This contradicts the condition that $v$ is not the zero function in $D$. 
Step 3: If there exists a point $x_{0}^{\prime} \in \partial D$ such that

$$
u\left(x_{0}^{\prime}\right)=K v\left(x_{0}^{\prime}\right)=0,
$$

then we have, by Claims 2.1 and 2.2 ,

$$
\left\{\begin{array}{l}
A u(x)=v(x) \geq 0 \text { for all } x \in D \\
u\left(x_{0}^{\prime}\right)=\min _{\bar{D}} u=0 \\
u(x)>0 \text { for all } x \in D
\end{array}\right.
$$

Thus it follows from an application of the Hopf boundary point lemma (Lemma A.1) that

$$
\frac{\partial u}{\partial \boldsymbol{\nu}}\left(x_{0}^{\prime}\right)<0
$$

so that

$$
a\left(x_{0}^{\prime}\right)=0
$$

since we have

$$
0=B u\left(x_{0}^{\prime}\right)=a\left(x_{0}^{\prime}\right) \frac{\partial u}{\partial \nu}\left(x_{0}^{\prime}\right)=0 .
$$

Conversely, if $a\left(x_{0}^{\prime}\right)=0$, then we have, by condition (H.2),

$$
b\left(x_{0}^{\prime}\right)>0,
$$

and so

$$
u\left(x_{0}^{\prime}\right)=0
$$

since $0=B u\left(x_{0}^{\prime}\right)=b\left(x_{0}^{\prime}\right) u\left(x_{0}^{\prime}\right)$.

Summing up, we have proved that

$$
\left\{\begin{array}{l}
u\left(x^{\prime}\right)=0 \Longleftrightarrow x^{\prime} \in M, \\
u(x)>0 \Longleftrightarrow x \in \bar{D} \backslash M .
\end{array}\right.
$$

This proves the desired assertions (a) and (b).

Assertion (c) is an immediate consequence of the Hopf boundary point lemma (Lemma A.1), since the function $u$ attains its minimum 0 at the set $M$.

Finally, in order to prove the positivity of $K: C(\bar{D}) \rightarrow C(\bar{D})$, let $v$ be an arbitrary function in $C(\bar{D})$ such that $v \geq 0$ and $v \not \equiv 0$ on $\bar{D}$. Then, by using Friedrichs' mollifiers we can find a sequence $\left\{v_{j}\right\} \subset C^{1}(\bar{D})$ satisfying the conditions

$$
\begin{cases}v_{j} \geq 0 & \text { on } \bar{D} \\ v_{j} \longrightarrow v & \text { in } C(\bar{D}) .\end{cases}
$$

Hence we have, by assertions (a) and (b),

$$
\begin{cases}K v_{j} \in C^{2}(\bar{D}), & \\ K v_{j} \geq 0 & \text { on } \bar{D} \\ K v_{j} \longrightarrow K v & \text { in } C(\bar{D}) .\end{cases}
$$

This proves that

$$
K v \geq 0 \text { on } \bar{D} \text {. }
$$

The proof of Lemma 2.2 is complete. 
If we let

$$
e(x):=(K 1)(x)
$$

it follows from an application of Lemma 2.2 that the function $e \in C^{2+\theta}(\bar{D})$ satisfies the conditions

$$
\begin{cases}e\left(x^{\prime}\right)=0 & \text { on } M \\ e(x)>0 & \text { on } \bar{D} \backslash M \\ \frac{\partial e}{\partial \boldsymbol{\nu}}\left(x^{\prime}\right)<0 & \text { on } M\end{cases}
$$

By using the function $e=K 1$, we introduce a subspace of $C(\bar{D})$ as follows:

$$
\begin{aligned}
& C_{e}(\bar{D}) \\
= & \{u \in C(\bar{D}): \text { there is a constant } c>0 \text { such that }-c e \leq u \leq c e \text { on } \bar{D}\} \\
= & \bigcup_{c>0}[-c e, c e] .
\end{aligned}
$$

The space $C_{e}(\bar{D})$ is given a norm by the formula

$$
\|u\|_{e}=\inf \{c>0:-c e \leq u \leq c e \text { on } \bar{D}\} .
$$

By rescaling, we may assume that

$$
\|e\|_{C(\bar{D})}=\max _{x \in \bar{D}} e(x)=1 .
$$

Then we have the inequality

$$
-c e(x) \leq v(x) \leq c e(x) \text { on } \bar{D} \text { for all } v \in C_{e}(\bar{D}),
$$

and so

$$
\|v\|_{C(\bar{D})} \leq c\|e\|_{C(\bar{D})}=c \quad \text { for all } v \in C_{e}(\bar{D}) .
$$

This proves that

$$
\|v\|_{C(\bar{D})} \leq\|v\|_{e} \quad \text { for all } v \in C_{e}(\bar{D}) .
$$

Namely, the injection

$$
C_{e}(\bar{D}) \hookrightarrow C(\bar{D})
$$

is continuous.

If we let

$$
P_{e}=C_{e}(\bar{D}) \cap P=\left\{u \in C_{e}(\bar{D}): u \geq 0 \text { on } \bar{D}\right\},
$$

it is easy to verify that the space $C_{e}(\bar{D})$ is an ordered Banach space having the positive cone $P_{e}$ with nonempty interior.

The next proposition will play an important role in the proof of Theorem 1.2 (see [70, Proposition 2.2]):

Proposition 2.2 The operator $K$ maps $C(\bar{D})$ compactly into $C_{e}(\bar{D})$ :

$$
K: C(\bar{D}) \underset{\text { compactly }}{\longrightarrow} C_{e}(\bar{D}) .
$$

Moreover, $K$ is strongly positive, that is, if $v \in P \backslash\{0\}$, then the function $K v$ is an interior point of $P_{e}$ :

$$
v \in P \backslash\{0\} \Longrightarrow K v \in \operatorname{Int}\left(P_{e}\right) .
$$


Proof The proof is divided into three steps.

Step (i): First, by the positivity of $K$ we find that $K$ maps $C(\bar{D})$ into $C_{e}(\bar{D})$. Indeed, we have, for all $v \in C(\bar{D})$,

$$
-\|v\|_{C(\bar{D})} \leq v(x) \leq\|v\|_{C(\bar{D})} \quad \text { on } \bar{D} .
$$

Hence it follows from the positivity of $K$ that

$$
-\|v\|_{C(\bar{D})} \cdot K 1(x) \leq K v(x) \leq\|v\|_{C(\bar{D})} \cdot K 1(x) \quad \text { on } \bar{D} .
$$

This proves that

$$
-c e \leq K v \leq c e \text { on } \bar{D}
$$

with $c=\|v\|_{C(\bar{D})}$.

Step (ii): Next we prove that

$$
K: C(\bar{D}) \longrightarrow C_{e}(\bar{D})
$$

is compact. To do this, we let

$$
C_{B}^{1}(\bar{D})=\left\{u \in C^{1}(\bar{D}): B u=0 \text { on } \partial D\right\} .
$$

Since $K$ maps $C(\bar{D})$ compactly into $C_{B}^{1}(\bar{D})$, it suffices to show that the inclusion mapping

$$
\iota: C_{B}^{1}(\bar{D}) \longrightarrow C_{e}(\bar{D})
$$

is continuous. The situation can be visualized as follows (see also Figure 2.1):

$$
K: C(\bar{D}) \underset{\text { compactly }}{\hookrightarrow \hookrightarrow} C_{B}^{1}(\bar{D}) \stackrel{\iota}{\longrightarrow} C_{e}(\bar{D}) .
$$

(ii-a) We verify that $\iota$ maps $C_{B}^{1}(\bar{D})$ into $C_{e}(\bar{D})$. Let $u$ be an arbitrary function in $C_{B}^{1}(\bar{D})$. Since we have, for some neighborhood $\omega$ of $M$ in $\partial D$,

$$
\begin{cases}b\left(x^{\prime}\right)>0 & \text { in } \omega \\ \frac{\partial e}{\partial \boldsymbol{\nu}}\left(x^{\prime}\right)<0 & \text { in } \omega\end{cases}
$$

it follows that

$$
\frac{u}{e}=\frac{\left(-\frac{a\left(x^{\prime}\right)}{b\left(x^{\prime}\right)}\right) \frac{\partial u}{\partial \boldsymbol{\nu}}}{\left(-\frac{a\left(x^{\prime}\right)}{b\left(x^{\prime}\right)}\right) \frac{\partial e}{\partial \boldsymbol{\nu}}}=\frac{\frac{\partial u}{\partial \boldsymbol{\nu}}}{\frac{\partial e}{\partial \boldsymbol{\nu}}} \quad \text { in } \omega \backslash M
$$

Hence there exists a constant $c_{1}>0$ such that

$$
\left|u\left(x^{\prime}\right)\right| \leq c_{1} e\left(x^{\prime}\right) \quad \text { in } \omega .
$$

Thus, by using Taylor's formula we can find a neighborhood $W$ of $\omega$ in $D$ and a constant $c_{2}>0$ such that

$$
|u(x)| \leq c_{2} e(x) \quad \text { in } W .
$$

On the other hand, since we have, for some constant $\alpha>0$,

$$
e(x) \geq \alpha \quad \text { on } \bar{D} \backslash W,
$$


we can find a constant $c_{3}>0$ such that

$$
\left|\frac{u(x)}{e(x)}\right| \leq c_{3} \quad \text { on } \bar{D} \backslash W .
$$

Therefore we have, with $c=\max \left\{c_{2}, c_{3}\right\}$,

$$
-c e(x) \leq u(x) \leq c e(x) \text { on } \bar{D} \text {. }
$$

This proves that $u \in C_{e}(\bar{D})$.

(ii-b) Now we show that the inclusion mapping

$$
\iota: C_{B}^{1}(\bar{D}) \longrightarrow C_{e}(\bar{D})
$$

is continuous. To do so, we have only to prove the closedness of $\iota$, since its continuity follows immediately from an application of Banach's closed graph theorem ([74, Chapter II, Section 6, Theorem 1]).

We assume that

$$
\begin{cases}u_{j} \in C_{B}^{1}(\bar{D}), & \\ u_{j} \longrightarrow u & \text { in } C_{B}^{1}(\bar{D}), \\ u_{j} \longrightarrow v & \text { in } C_{e}(\bar{D}) .\end{cases}
$$

Then there exists a sequence $\left\{c_{j}\right\}, c_{j} \rightarrow 0$, such that

$$
\left\|u_{j}-v\right\|_{e} \leq c_{j}\|e\| \text {. }
$$

Hence we have the assertion

$$
u_{j} \longrightarrow v \quad \text { in } C(\bar{D})
$$

and so

$$
\iota(u)=u=v .
$$

This proves the closedness of $\iota$.

Step (iii) It remains to prove the strong positivity of $K$, that is, $K(P \backslash\{0\}) \subset$ Int $\left(P_{e}\right)$.

(iii-a) We show that, for every $v \in P \backslash\{0\}$ there exist constants $\beta>0$ and $\gamma>0$ such that

$$
\beta e(x) \leq K v(x) \leq \gamma e(x) \text { on } \bar{D} .
$$

By the positivity of $K$, we may modify the function $v$ in such a way that $v \in C^{1}(\bar{D})$. Furthermore, since the functions $u=K v$ and $e=K 1$ vanish only on the set

$$
M=\left\{x^{\prime} \in \partial D: a\left(x^{\prime}\right)=0\right\},
$$

it suffices to prove that there exists a neighborhood $W$ of $M$ in $D$ such that

$$
\beta e(x) \leq u(x) \text { in } W .
$$

We recall (see Lemma 2.2) that we have, in a neighborhood $\omega$ of $M$ in $\partial D$,

$$
\begin{cases}u=\left(-\frac{a\left(x^{\prime}\right)}{b\left(x^{\prime}\right)}\right) \frac{\partial u}{\partial \boldsymbol{\nu}} & \text { in } \omega, \\ \frac{\partial u}{\partial \boldsymbol{\nu}}<0 & \text { in } \omega,\end{cases}
$$


and

$$
\begin{cases}e=\left(-\frac{a\left(x^{\prime}\right)}{b\left(x^{\prime}\right)}\right) \frac{\partial e}{\partial \boldsymbol{\nu}} & \text { in } \omega \\ \frac{\partial e}{\partial \boldsymbol{\nu}}<0 & \text { in } \omega .\end{cases}
$$

Thus we have, for $\beta$ sufficiently small,

$$
\begin{cases}u\left(x^{\prime}\right)-\beta e\left(x^{\prime}\right) \geq 0 & \text { in } \omega, \\ \frac{\partial}{\partial \boldsymbol{\nu}}(u-\beta e)\left(x^{\prime}\right)<0 & \text { in } \omega .\end{cases}
$$

Therefore, by using Taylor's formula we can find a neighborhood $W$ of $M$ in $D$ such that

$$
u(x)-\beta e(x) \geq 0 \quad \text { in } W .
$$

This proves the desired inequality (2.8).

(iii-b) Finally, we show that the function $u=K v$ is an interior point of $P_{e}$. Take

$$
\varepsilon=\frac{\beta}{2},
$$

where $\beta$ is the same constant as in estimate (2.7). Then, for all functions $w \in C_{e}(\bar{D})$ satisfying

$$
\|w-K v\|_{e}<\varepsilon,
$$

we have, by estimate $(2.7)$,

$$
w \leq K v+\varepsilon e \leq(\gamma+\varepsilon) e,
$$

and also

$$
w \geq K v-\varepsilon e \geq \frac{\beta}{2} e=\varepsilon e \text { on } \bar{D} .
$$

This implies that

$$
B_{e}(K v, \varepsilon):=\left\{w \in C_{e}(\bar{D}):\|w-K v\|_{e}<\varepsilon\right\} \subset P_{e},
$$

that is, the function $K v$ is an interior point of $P_{e}$.

The proof of Proposition 2.2 is now complete.

The situation of Proposition 2.2 can be visualized as in Figure 2.2.

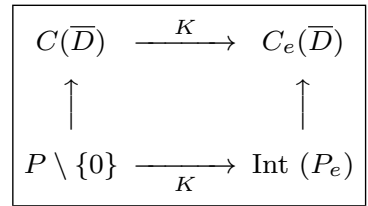

Fig. 2.2 The mapping properties of the resolvent $K$ in the ordered Banach spaces $C(\bar{D})$ and $C_{e}(\bar{D})$

Now we consider the resolvent $K$ as an operator in the ordered Banach space $C_{e}(\bar{D})$, and prove important results concerning its eigenfunctions and corresponding eigenvalues. 
First, Proposition 2.2 tells us that the operator

$$
K: C_{e}(\bar{D}) \longrightarrow C_{e}(\bar{D})
$$

is strongly positive and compact. This implies that $K$ has a countable number of positive eigenvalues $\mu_{j}$ which may accumulate only at 0 . Hence they may be arranged in a decreasing sequence

$$
\mu_{1} \geq \mu_{2} \geq \cdots \geq \mu_{j} \geq \cdots>0,
$$

where each eigenvalue is repeated according to its multiplicity.

The next theorem characterizes the eigenvalues and positive eigenfunctions of $K$ :

Theorem 2.6 The resolvent $K$, considered as an operator $K: C_{e}(\bar{D}) \rightarrow C_{e}(\bar{D})$, has the following spectral properties:

(i) The largest eigenvalue $\mu_{1}$ is algebraically simple, that is, $\mu_{1}>\mu_{2}$, and has a positive eigenfunction $\psi_{1}: K \psi_{1}=\mu_{1} \psi_{1}$.

(ii) No other eigenvalues, $\mu_{j}, j \geq 2$, have positive eigenfunctions.

This theorem is an immediate consequence of the Kre $\mathrm{n}-$ Rutman theorem (Theorem 2.5).

2.6 Proof of Theorem 2.1 - Part $2-$

By assertion (2.3) and Figure 2.2, it is easy to see that

$$
\begin{aligned}
\mathfrak{A} u=\lambda u \quad \text { in } L^{2}(D) & \Longleftrightarrow u=\lambda K u \quad \text { in } C(\bar{D}) \\
& \Longleftrightarrow K u=\frac{1}{\lambda} u \quad \text { in } C_{e}(\bar{D}) .
\end{aligned}
$$

Therefore, Theorem 2.1 (and hence Theorem 1.1) is an immediate consequence of Theorem 2.6.

\section{Elements of topological methods in nonlinear analysis}

This section is devoted to elements of topological methods in nonlinear analysis for the study of the semilinear boundary value problem (1.2). For detailed studies of topological methods in nonlinear analysis, the reader is referred to AmbrosettiMalchiodi [8], Ambrosetti-Prodi [13], Brown [22], Chang [23], Chow-Hale [25], Drábek-Milota [29], Nirenberg [43], Papageorgiou-Rădulescu-Repovš [44] and Zeidler [75].

First, by making use of bifurcation theory from a simple eigenvalue essentially due to Crandall-Rabinowitz [26] and Dancer [27], we discuss the changes that occur in the structure of the solutions of $F(\lambda, u)=0$ as $\lambda$ varies near the first eigenvalue $\lambda_{1}$ of the linear problem (1.1) (Theorems 3.3 and 3.4).

The Leray-Schauder degree is an important topological tool introduced by Leray-Schauder [39] in the study of the semilinear boundary value problem (1.2). The nontriviality of the degree guarantees the existence of a fixed point of the compact mapping in the domain (Theorem 3.6). 
3.1 Local properties of differentiable mappings

Let $X, Y$ be Banach spaces. Let $U$ be a subset of $X$. A map $f: U \rightarrow Y$ is said to be compact if it is continuous on $U$ and maps bounded sets in $U$ into relatively compact sets in $Y$.

Let $U$ be an open set in $X$ and let $f: U \rightarrow Y$ be a map. We say that the map $f$ is (Fréchet) differentiable at a point $x \in U$ if there exist a continuous linear operator $A: X \rightarrow Y$ and a map $\psi$ defined for all sufficiently small $h$ in $X$, with values in $Y$, such that

$$
\left\{\begin{array}{l}
f(x+h)=f(x)+A h+\|h\| \psi(h), \\
\lim _{h \rightarrow 0} \psi(h)=0 .
\end{array}\right.
$$

We remark that the continuous linear operator $A$ is uniquely determined by $f$ and $x$. The operator $A$ is called the (Fréchet) derivative of $f$ at $x$, and is denoted by $D f(x)$ or $f^{\prime}(x)$. A map $f$ is said to be (Fréchet) differentiable on $U$ if it is (Fréchet) differentiable at every point of $U$. In this case, the derivative $D f$ is a map of $U$ into the Banach space $B(X, Y)$ of all continuous (bounded) linear operators:

$$
\begin{aligned}
D f: & U \longrightarrow B(X, Y) \\
u & \longmapsto D f(u) .
\end{aligned}
$$

If in addition $D f$ is continuous from $U$ into $B(X, Y)$, we say that $f$ is of class $C^{1}$.

If the derivative $D f$ is differentiable at a point $x \in U$ (resp. in $U$ ), we say that $f$ is twice differentiable at $x$ (resp. in $U$ ). The derivative of $D f$ at $x$ is called the second derivative of $f$ at $x$, and is denoted by $D^{2} f(x)$. This is an element of the Banach space $B(X, B(X, Y))$ which can be naturally identified with the space $B_{2}(X, Y)=B(X, X ; Y)$ of all continuous bilinear mappings of $X \times X$ into $Y$.

By induction on $k$, we define a $k$ times differentiable mapping $f$ of $U$ into $Y$ as a $(k-1)$ times differentiable mapping whose $(k-1)$-th derivative $D^{k-1} f$ is differentiable in $U$. The derivative $D^{k} f=D\left(D^{k-1} f\right)$ is called the $k$-th derivative of $f$. The derivative $D^{k} f(x)$ at a point $x \in U$ can be identified with an element of the space $B_{k}(X, Y)$ of all continuous $k$-linear mappings of $X \times \cdots \times X$ into $Y$. A map $f: U \rightarrow Y$ is said to be of class $C^{r}(r \geq 2)$ in $U$ if all the derivatives $D^{k} f$ exist and are continuous in $U$ for $1 \leq k \leq r$.

Here it is worthwhile pointing out that if $X=\mathbf{R}$, then the space $B(X, Y)$ can be identified with the space $Y$, so the space $B_{k}(\mathbf{R}, Y)$ can be identified with the space $Y$ for general $k \geq 2$.

Now we assume that the Banach space $X$ is the product space of two Banach spaces $X_{1}$ and $X_{2}$ :

$$
X=X_{1} \times X_{2} .
$$

For each point $x=\left(x_{1}, x_{2}\right) \in U \subset X$, one can consider the partial mappings

$$
\begin{aligned}
& F_{1}: u_{1} \longmapsto f\left(u_{1}, x_{2}\right), \\
& F_{2}: u_{2} \longmapsto f\left(x_{1}, u_{2}\right)
\end{aligned}
$$

of open subsets of $X_{1}$ and $X_{2}$ respectively into $Y$. We say that $f$ is differentiable with respect to the first (resp. second) variable if the mapping $F_{1}\left(u_{1}\right)$ (resp. $F_{2}\left(u_{2}\right)$ ) is differentiable at $x_{1}$ (resp. at $\left.x_{2}\right)$. The derivative $D F_{1}\left(x_{1}\right)\left(\operatorname{resp} . D F_{2}\left(x_{2}\right)\right)$ is an element of the Banach space $B\left(X_{1}, Y\right)$ (resp. $B\left(X_{2}, Y\right)$ ), and is called the partial 
(Fréchet) derivative of $f$ at $\left(x_{1}, x_{2}\right)$ with respect to the first (resp. second) variable. We write

$$
\begin{aligned}
& D_{x_{1}} f\left(x_{1}, x_{2}\right)=D F_{1}\left(x_{1}\right), \\
& D_{x_{2}} f\left(x_{1}, x_{2}\right)=D F_{2}\left(x_{2}\right) .
\end{aligned}
$$

We can define inductively the partial (Fréchet) derivatives $D_{x_{1}}^{k} D_{x_{2}}^{\ell} f$ for general $k$ and $\ell$.

The process of linearization provides a key link between the linear and nonlinear theories of partial differential equations. Our basic tool is the implicit function theorem (cf. [43, Theorem 2.7.2], [23, Theorem 1.2.1], [29, Theorem 4.2.1]):

Theorem 3.1 (the implicit function theorem) Let $X, Y, Z$ be Banach spaces, and let $f$ be a $C^{r}$ map $(r \geq 1)$ of an open subset $U \times V$ of $X \times Y$ into $Z$. Assume that the derivative $D_{y} f\left(x_{0}, y_{0}\right): Y \rightarrow Z$ is an algebraic and topological isomorphism at a point $\left(x_{0}, y_{0}\right)$ of $U \times V$. Then there exist neighborhoods $U_{0}$ of $x_{0}$ and $W_{0}$ of $f\left(x_{0}, y_{0}\right)$ and a unique $C^{r}$ map $g: U_{0} \times W_{0} \rightarrow V$ such that

$$
f(x, g(x, w))=w \quad \text { for all }(x, w) \in U_{0} \times W_{0} .
$$

The local inverse mapping theorem provides a criterion for a map to be a local $C^{r}$ diffeomorphism in terms of its derivative (see [43, Corollary 2.7.3], [23, Theorem 1.2.3], [29, Theorem 4.1.1]):

Theorem 3.2 (the local inverse mapping theorem) Let $X, Y$ be Banach spaces, and let $f$ be a $C^{r}$ map $(r \geq 1)$ of an open subset $U$ of $X$ into $Y$. Assume that the derivative $f^{\prime}\left(x_{0}\right): X \rightarrow Y$ is an algebraic and topological isomorphism at a point $x_{0}$ of $U$. Then the map $f$ is a $C^{r}$ diffeomorphism of some neighborhood of $x_{0}$ onto some neighborhood of $f\left(x_{0}\right)$.

3.2 Bifurcation theory from a simple eigenvalue

Now we study the operator equation of the following form

$$
f(\lambda, x)=0,
$$

where $f$ depends on a real parameter $\lambda$. In other words, $f(\lambda, x)$ is a nonlinear operator, depending on the parameter $\lambda$, which operates on the unknown vector $x$. One of the first questions to be answered is whether or not the equation $f(\lambda, x)=0$ has any solution $x$ for a given value of $\lambda$. If it does, the question of how many solutions it has arises, and then how this number varies with $\lambda$. Of particular interest is the process of bifurcation whereby a given solution of $f(\lambda, x)=0$ splits into two or more solutions as $\lambda$ passes through some critical value $\lambda_{1}$.

Now let $f(\lambda, x)$ be a $C^{k}$ map, $k \geq 3$, of a neighborhood of $\left(\lambda_{1}, 0\right)$ in a Banach space $\mathbf{R} \times X$ into a Banach space $Y$ such that

$$
f\left(\lambda_{1}, 0\right)=0 .
$$

The point $\left(\lambda_{1}, 0\right)$ is called a bifurcation point of the operator equation $f(\lambda, x)=0$ if every neighborhood of $\left(\lambda_{1}, 0\right)$ in $\mathbf{R} \times X$ contains a solution $(\lambda, x)$ with $x \neq 0$.

The next theorem gives sufficient conditions in order that the point $\left(\lambda_{1}, 0\right)$ is a bifurcation point of the map $f$ (cf. [26, Theorem 1.7], [29, Chapter 4, Theorem 4.3.22], [43, Theorem 3.2.2]): 
Theorem 3.3 (Crandall-Rabinowitz) Let $f(\lambda, x)$ be a $C^{k}$ map, $k \geq 3$, of a neighborhood of $\left(\lambda_{1}, 0\right)$ in a Banach space $\mathbf{R} \times X$ into a Banach space $Y$ such that

$$
f\left(\lambda_{1}, 0\right)=0
$$

Assume that the following four conditions are satisfied:

(i) $D_{\lambda} f\left(\lambda_{1}, 0\right)=0$.

(ii) The null space $N\left(D_{x} f\left(\lambda_{1}, 0\right)\right)$ is one-dimensional, spanned by a vector $x_{0}$.

(iii) The range $R\left(D_{x} f\left(\lambda_{1}, 0\right)\right)$ has codimension one in the space $Y$.

(iv) $D_{\lambda}^{2} f\left(\lambda_{1}, 0\right) \in R\left(D_{x} f\left(\lambda_{1}, 0\right)\right)$ and $D_{\lambda} D_{x} f\left(\lambda_{1}, 0\right) x_{0} \notin R\left(D_{x} f\left(\lambda_{1}, 0\right)\right)$.

Then the point $\left(\lambda_{1}, 0\right)$ is a bifurcation point of the operator equation (3.1). In fact, the set of solutions of $f(\lambda, x)=0$ near $\left(\lambda_{1}, 0\right)$ consists of two $C^{k-2}$ curves $\Gamma_{1}$ and $\Gamma_{2}$ intersecting only at the point $\left(\lambda_{1}, 0\right)$. Furthermore, the curve $\Gamma_{1}$ is tangent to the $\lambda$-axis at $\left(\lambda_{1}, 0\right)$ and may be parametrized by $\lambda$ as

$$
\Gamma_{1}=\left\{\left(\lambda, x_{1}(\lambda)\right):\left|\lambda-\lambda_{1}\right|<\varepsilon\right\},
$$

while the curve $\Gamma_{2}$ may be parametrized by a variable $s$ as

$$
\Gamma_{2}=\left\{\left(\lambda_{2}(s), s x_{0}+x_{2}(s)\right):|s|<\varepsilon\right\} .
$$

Here

$$
x_{2}(0)=\frac{d x_{2}}{d s}(0)=0, \quad \lambda_{2}(0)=\lambda_{1} .
$$

In the case $f(\lambda, 0)=0$ for all $\left|\lambda-\lambda_{1}\right|$ sufficiently small, we have a trivial solution $x=0$ available; so the curve $\Gamma_{1}$ is in the $\lambda$-axis (see Figure 3.1).

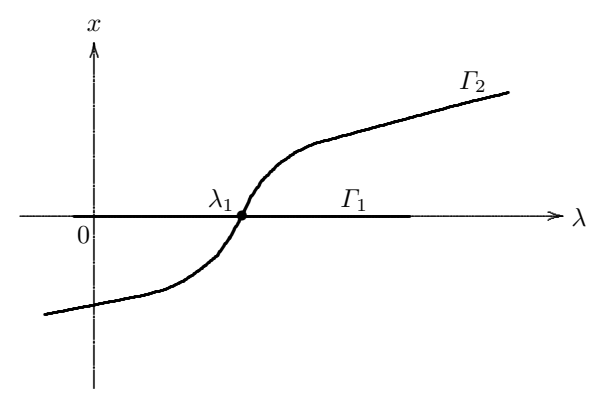

Fig. 3.1 The bifurcation curves $\Gamma_{1}$ and $\Gamma_{2}$ in the case $f(\lambda, 0)=0$

The next theorem asserts the existence of global solution branches for positive mappings due to Dancer [27, Corollary to Theorem 2] (cf. Rabinowitz [47, Theorem 1.3], Amann [5, Theorem 18.3]):

Theorem 3.4 (Dancer) Let $E$ be an ordered Banach space with total positive cone P. Let

$$
A: \mathbf{R} \times E \longrightarrow E
$$


be a completely continuous map that satisfies the conditions

$$
\begin{cases}A(0, x)=0 & \text { for } x \in E, \\ A(\lambda, 0)=0 & \text { for } \lambda \in \mathbf{R} .\end{cases}
$$

Assume that there exist a linear operator $B: E \rightarrow E$ and a map $F: \mathbf{R} \times E \rightarrow E$ such that

$$
A(\lambda, x)=\lambda B x+F(\lambda, x)
$$

where

$$
\|F(\lambda, x)\|=o(\|x\|) \quad \text { as } x \rightarrow 0 \text { in } E \text {, locally uniformly in } \lambda \in \mathbf{R} \text {. }
$$

We let

$C_{P}(B):=\{\lambda \in[0, \infty):$ There exists an $x \in P$ with $\|x\|=1$ such that $\lambda B x=x\}$.

If the spectral radius $r(B)$ is positive, then the component of the set

$$
\mathcal{D}_{P}(A)=\{(\lambda, x) \in[0, \infty) \times P: x=A(\lambda, x), x \neq 0\} \cup\left(\{0\} \times C_{P}(B)\right)
$$

containing $(1 / r(B), 0)$ is unbounded in $\mathbf{R} \times E$. Moreover, the point $(1 / r(B), 0)$ is a bifurcation point of the nonlinear operator equation $x=A(\lambda, x)$ to the trivial solution (see Figure 3.2).

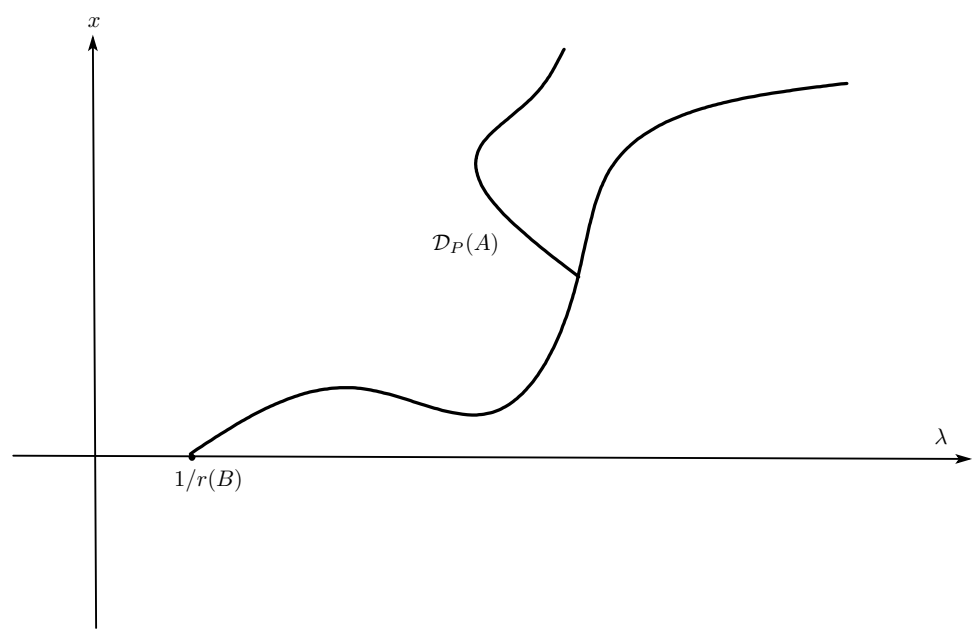

Fig. 3.2 The point $(1 / r(B), 0)$ is a bifurcation point of the nonlinear operator equation $x=$ $A(\lambda, x)$ to the trivial solution

Remark 3.1 Some remarks are in order:

(i) The operator $B: E \rightarrow E$ is compact. Hence the set $C_{P}(B)$ is a countable set with no finite limit point.

(ii) If $P$ has non-empty interior $\operatorname{Int}(P)$ and if $\mathrm{B}$ is a strongly positive, then it follows from an application of the Kreln-Rutman theorem (Theorem 2.4) that $r(T)>0$. Hence, the sharp version of the Krel̆n-Rutman theorem (Theorem 2.5) applies. 
3.3 Brouwer degree

In this subsection we consider the following situation:

(a) $\Omega$ is a bounded open set in $\mathbf{R}^{n}$ with boundary $\partial \Omega$.

(b) $f=\left(f_{1}, \ldots, f_{n}\right): \bar{\Omega} \rightarrow \mathbf{R}^{n}$ is a continuous map.

(c) $p$ is a point of $\mathbf{R}^{n}$ such that $f(x) \neq p$ for all $x \in \partial \Omega$.

For each triplet $(f, \Omega, p)$, we define an integer-valued function $\operatorname{deg}(f, \Omega, p)$ in three steps (see [22], [23], [29], [43], [75]):

(i) The regular value case: Let $f \in C^{1}\left(\Omega, \mathbf{R}^{n}\right) \cap C\left(\bar{\Omega}, \mathbf{R}^{n}\right)$. We say that $p$ is a regular value of $f$ if the Jacobian determinant

$$
J_{f}(x)=\operatorname{det}\left(\begin{array}{cccc}
\frac{\partial f_{1}}{\partial x_{1}} & \frac{\partial f_{1}}{\partial x_{2}} & \cdots & \frac{\partial f_{1}}{\partial x_{n}} \\
\frac{\partial f_{2}}{\partial x_{1}} & \frac{\partial f_{2}}{\partial x_{2}} & \cdots & \frac{\partial f_{2}}{\partial x_{n}} \\
\cdot & \cdot & \cdots & \cdot \\
\cdot & \cdot & \cdots & \cdot \\
\frac{\partial f_{n}}{\partial x_{1}} & \frac{\partial f_{n}}{\partial x_{2}} & \cdots & \frac{\partial f_{n}}{\partial x_{n}}
\end{array}\right)
$$

is different from zero for every $x \in f^{-1}(p)$, where

$$
f^{-1}(p)=\{x \in \Omega: f(x)=p\} .
$$

It is easy to see that if $p$ is a regular value of $f$, then the set $f^{-1}(p)$ is a finite set. Thus we can define an integer $\operatorname{deg}(f, \Omega, p)$ for any regular value $p$ of $f$ by the formula

$$
\operatorname{deg}(f, \Omega, p)= \begin{cases}\sum_{x \in f^{-1}(p)} \operatorname{sgn} J_{f}(x) & \text { if } f^{-1}(p) \neq \emptyset, \\ 0 & \text { if } f^{-1}(p)=\emptyset .\end{cases}
$$

The integer $\operatorname{deg}(f, \Omega, p)$ is called the Brouwer degree of the map $f$ with respect to the set $\Omega$ and the point $p$.

(ii) The singular value case: Let $f \in C^{1}\left(\Omega, \mathbf{R}^{n}\right) \cap C\left(\bar{\Omega}, \mathbf{R}^{n}\right)$. In order to define the Brouwer degree for any value $p$ of $f$, we make use of the following theorem:

Theorem 3.5 (Sard) Let $f \in C^{1}\left(\Omega, \mathbf{R}^{n}\right)$. If we define the set $S_{f}$ of singular points of $f$ by the formula

$$
S_{f}=\left\{x \in \Omega: J_{f}(x)=0\right\},
$$

then the Lebesgue measure of $f\left(S_{f}\right)$ is equal to zero.

If $p$ is a point of $f\left(S_{f}\right)$, by virtue of Sard's theorem we can find a sequence $\left\{p_{j}\right\}$ of points in $\mathbf{R}^{n}$ such that

$$
\begin{cases}p_{j} \notin f\left(S_{f}\right), & \\ p_{j} \longrightarrow p & \text { as } j \rightarrow \infty, \\ p_{j} \neq f(x) & \text { for each } x \in \partial \Omega \text { and all } j \in \mathbf{N}\end{cases}
$$

Then we define the degrees $\operatorname{deg}\left(f, \Omega, p_{j}\right)$ by formula (3.2). We can show that the limit

$$
\lim _{j \rightarrow \infty} \operatorname{deg}\left(f, \Omega, p_{j}\right)
$$

exists and is independent of the approximation sequence $\left\{p_{j}\right\}$ chosen. 
Thus we can define the Brouwer degree for any value $p$ of $f$ as follows:

$$
\operatorname{deg}(f, \Omega, p)=\lim _{j \rightarrow \infty} \operatorname{deg}\left(f, \Omega, p_{j}\right)
$$

(iii) The general case: Let $f \in C\left(\bar{\Omega}, \mathbf{R}^{n}\right)$. By using the Weierstrass approximation theorem, we can find a sequence $\left\{f_{k}\right\}$ of polynomials such that

$$
\begin{cases}f_{k} \longrightarrow f & \text { uniformly on } \bar{\Omega} \text { as } k \rightarrow \infty, \\ p \neq f_{k}(x) & \text { for each } x \in \partial \Omega \text { and all } k \in \mathbf{N} .\end{cases}
$$

Then we define the degrees $\operatorname{deg}\left(f_{k}, \Omega, p\right)$ by formula (3.3). We can show that the limit

$$
\lim _{k \rightarrow \infty} \operatorname{deg}\left(f_{k}, \Omega, p\right)
$$

exists and is independent of the approximation sequence $\left\{f_{k}\right\}$ chosen.

In this way, for any map $f \in C\left(\bar{\Omega}, \mathbf{R}^{n}\right)$ and any point $p \in \mathbf{R}^{n} \backslash f(\partial \Omega)$ we can define the Brouwer degree, $\operatorname{deg}(f, \Omega, p)$, by the formula

$$
\operatorname{deg}(f, \Omega, p)=\lim _{k \rightarrow \infty} \operatorname{deg}\left(f_{k}, \Omega, p\right)
$$

The Brouwer degree enjoys some basic properties (see [22, Chapter 9]):

(i) (Normalization property): If $I: \bar{\Omega} \rightarrow \mathbf{R}^{n}$ is the identity map and $p \in \mathbf{R}^{n}$, then we have the formula

$$
\operatorname{deg}(I, \Omega, p)=\left\{\begin{array}{ll}
1 & \text { if } p \in \Omega \\
0 & \text { if } p \notin \Omega .
\end{array} .\right.
$$

(ii) (Translation invariance property): $\operatorname{deg}(f, \Omega, p)=\operatorname{deg}(f-p, \Omega, 0)$.

(iii) (Domain additivity property): If $\Omega_{1}, \Omega_{2}$ are disjoint open subsets of $\Omega$ and a point $p \in \Omega$ such that $p \notin f\left(\bar{\Omega} \backslash\left(\Omega_{1} \cup \Omega_{2}\right)\right)$, then we have the formula

$$
\operatorname{deg}(f, \Omega, p)=\operatorname{deg}\left(\left.f\right|_{\overline{\Omega_{1}}}, \Omega_{1}, p\right)+\operatorname{deg}\left(\left.f\right|_{\overline{\Omega_{2}}}, \Omega_{2}, p\right) .
$$

Here $\left.f\right|_{V}$ is the restriction of $f$ to $V$.

(iv) (Solution property): If $\operatorname{deg}(f, \Omega, p) \neq 0$, then there exists a point $x \in \Omega$ such that

$$
f(x)=p .
$$

(v) (Excision property): Let $\Omega_{0}$ be an open subset of $\Omega$ such that

$$
f(x) \neq p \quad \text { for all } x \in \Omega \backslash \Omega_{0},
$$

then we have the formula

$$
\operatorname{deg}(f, \Omega, p)=\operatorname{deg}\left(f, \Omega_{0}, p\right) .
$$

(vi) (Homotopy invariance property): For every family $h(\cdot, t): \bar{\Omega} \times[0,1] \rightarrow \mathbf{R}^{n}$ of compact maps such that $h(x, t) \neq p$ for all $(x, t) \in \partial \Omega \times[0,1]$, the integer

$$
\operatorname{deg}(h(\cdot, t), \Omega, p)
$$

is independent of $t \in[0,1]$. 
3.4 The Leray-Schauder degree

The Leray-Schauder degree is an extension of the Brouwer degree to mappings defined on an infinite dimensional real Banach space. The Brouwer fixed-point theorem asserts that a continuous map $f$ of a closed, bounded convex set $K \subset \mathbf{R}^{n}$ into itself has a fixed point. This is no longer true in infinite dimensions. In infinitedimensional spaces, we must require more of $f$ than mere continuity (see [22], [23], [29], [43], [75]).

Let $X$ be a real Banach space and let $\Omega$ be a bounded and open subset of $X$ with boundary $\partial \Omega$. A continuous map

$$
f: \Omega \longrightarrow X
$$

is said to be compact if it maps bounded sets in $\Omega$ into relatively compact sets of $X$. It should be emphasized that $f: \Omega \rightarrow X$ is compact if and only if $f$ is a uniform limit of mappings whose ranges lie in finite-dimensional subspaces.

The Leray-Schauder degree $\operatorname{deg}(f, \Omega, p)$ of a compact perturbation $f=I-K$ of the identity map $I$ at a point $p \in X$ and relative to $\Omega$ can be defined by an analogue of the Galerkin approximation procedures, by assuming that

$$
f(x) \neq p \quad \text { on } \partial \Omega .
$$

More precisely, we define the integer-valued function $\operatorname{deg}(f, \Omega, p)$ in two steps (see [22, Chapter 10]):

Step 1: If the compact mapping $K: \Omega \rightarrow X$ has finite-dimensional range contained in a finite-dimensional subspace $X_{n}$ of $X$, then, by assuming that $p \in X_{n}$ we define the Leray-Schauder degree of $I-K$ at $p$ relative $\Omega, \operatorname{deg}(I-K, \Omega, p)$, by the formula

$$
\operatorname{deg}(I-K, \Omega, p)=\operatorname{deg}\left(I-K, \Omega \cap X_{n}, p\right) .
$$

We can verify that the integer $\operatorname{deg}\left(I-K, \Omega \cap X_{n}, p\right)$ is independent of the finitedimensional subspace $X_{n}$ containing $p$ and the range of $K$.

Step 2: For a general compact mapping $K: \Omega \rightarrow X$, we approximate $K$ by a sequence $\left\{K_{n}\right\}$ of compact mappings with finite-dimensional range $K_{n}: \Omega \rightarrow X_{n}$ such that

$$
\sup _{x \in \bar{\Omega}}\left\|K x-K_{n} x\right\| \leq \frac{1}{n} .
$$

Then, by assuming that $p \in X_{n}$ as in Step 1 we define the Leray-Schauder degree, $\operatorname{deg}(I-K, \Omega, p)$, by the formula

$$
\operatorname{deg}(I-K, \Omega, p)=\lim _{n \rightarrow \infty} \operatorname{deg}\left(I-K_{n}, \Omega \cap X_{n}, p\right) .
$$

We can show that the limit (3.4) exists and is independent of the approximating sequence $\left\{K_{n}\right\}$.

Similar to the Brouwer degree, the Leray-Schauder degree enjoys some basic properties (see [22, Chapter 11]):

(I) Normalization property: If $I: X \rightarrow X$ is the identity map, then we have the assertion

$$
\operatorname{deg}(I, \Omega, p)= \begin{cases}1 & \text { if } p \in \Omega \\ 0 & \text { if } p \notin \Omega\end{cases}
$$


(II) Homotopy invariance property: Let $\Omega$ be a bounded and open subset of $X$. If $K: \bar{\Omega} \times[0,1] \rightarrow X$ is compact and if a point $p \in X$ satisfies the condition

$$
x-K(x, t) \neq p \quad \text { for all } x \in \partial \Omega \text { and } 0 \leq t \leq 1,
$$

then it follows that $\operatorname{deg}(I-K(\cdot, t), \Omega, p)$ is independent of $t$.

(III) Translation invariance property: For each point $p \in X$, we have the formula

$$
\operatorname{deg}(I-K, \Omega, p)=\operatorname{deg}(I-K-p, \Omega, 0) .
$$

(IV) Domain additivity property: If $\Omega_{1}$ and $\Omega_{2}$ are two open subsets in $\Omega$ such that $\Omega_{1} \cap \Omega_{2}=\emptyset$ and satisfy the condition

$$
x-K x \neq p \quad \text { for all } x \in \bar{\Omega} \backslash\left(\Omega_{1} \cup \Omega_{2}\right),
$$

then we have the formula

$$
\operatorname{deg}(I-K, \Omega, p)=\operatorname{deg}\left(I-K, \Omega_{1}, p\right)+\operatorname{deg}\left(I-K, \Omega_{2}, p\right) .
$$

(V) Excision property: Let $\Omega$ be a bounded open set in $X$ such that

$$
x-K x \neq p \quad \text { for all } x \in \partial \Omega .
$$

If $W$ is an open subset of $\Omega$ such that

$$
x-K x \neq p \text { for all } x \in W,
$$

then we have the formula

$$
\operatorname{deg}(I-K, \Omega, p)=\operatorname{deg}(I-K, \Omega \backslash W, p) .
$$

(VI) Empty set property: $\operatorname{deg}(I-K, \emptyset, p)=0$.

The next theorem is a generalization of Kronecker's existence theorem for the Brouwer degree (see [23, Section 3.4]):

Theorem 3.6 (Kronecker) Let $\Omega$ be a bounded open subset of a real Banach space $X$ and let $K: \bar{\Omega} \rightarrow X$ be compact. If $p_{0} \notin(I-K)(\partial \Omega)$ and $\operatorname{deg}\left(I-K, \Omega, p_{0}\right) \neq 0$, then there exists a point $x_{0} \in \Omega$ such that $(I-K) x_{0}=p_{0}$.

Proof Our proof is based on a reduction to absurdity. Assume, to the contrary, that

$$
p_{0} \notin(I-K)(\bar{\Omega})=(I-K)(\Omega) \cup(I-K)(\partial \Omega) .
$$

Then we have, by the excision property (V) and the empty set property (VI),

$$
\operatorname{deg}\left(I-K, \Omega, p_{0}\right)=\operatorname{deg}\left(I-K, \Omega \backslash \bar{\Omega}, p_{0}\right)=\operatorname{deg}\left(I-K, \emptyset, p_{0}\right)=0 .
$$

This contradicts the assumption that $\operatorname{deg}\left(I-K, \Omega, p_{0}\right) \neq 0$.

The proof of Theorem 3.6 is complete. 
3.5 The Leray-Schauder index

Let $\phi$ be a mapping of the closure $\bar{\Omega}=\Omega \cup \partial \Omega$ into $X$ such that $\phi(x) \neq 0$ on $\partial \Omega$. Assume that

$$
\begin{aligned}
& \phi \in C^{1}(\Omega, X), \\
& K=I-\phi: \bar{\Omega} \longrightarrow X \quad \text { is compact. }
\end{aligned}
$$

If $x_{0}$ is an isolated solution of the equation $\phi(x)=0$ and if the Fréchet derivative $D \phi\left(x_{0}\right)=I-D K\left(x_{0}\right)$ at $x_{0}$ is invertible, then we can define the Leray-Schauder index of the map $\phi$ at $x_{0}, i\left(\phi, x_{0}, 0\right)$, by the formula

$$
i\left(\phi, x_{0}, 0\right)=\operatorname{deg}\left(\phi, B\left(x_{0}, \varepsilon\right), 0\right),
$$

where the open ball $B\left(x_{0}, \varepsilon\right)$ of radius $\varepsilon$ about $x_{0}$ is chosen so that it contains no other solution of the equation $\phi(x)=0$. It should be emphasized that the Leray-Schauder index $i\left(\phi, x_{0}, 0\right)$ does not depend on the special choice of $\varepsilon$.

The next index theorem plays an essential role in the proof of Theorem 8.2 in the resonance case (see [29, Proposition 5.8.11], [43, Theorem 2.8.1]):

Theorem 3.7 (the index theorem) Let $X$ be a real Banach space and let $\Omega$ be a bounded open set in $X$. If $\phi$ is a mapping of the closure $\bar{\Omega}=\Omega \cup \partial \Omega$ into $X$ such that $\phi(x) \neq 0$ on $\partial \Omega$ and if it satisfies the conditions

$$
\begin{aligned}
& \phi \in C^{1}(\Omega, X) \\
& K=I-\phi: \bar{\Omega} \longrightarrow X \quad \text { is compact }
\end{aligned}
$$

then we have the formula for the Leray-Schauder index $i\left(\phi, x_{0}, 0\right)$

$$
i\left(\phi, x_{0}, 0\right)=(-1)^{\beta},
$$

where

$$
\beta=\sum_{\mu_{j}>1} \beta_{j}
$$

$\beta_{j}$ being the algebraic multiplicity of the eigenvalue $\mu_{j}$ of the Fréchet derivative

$$
D K\left(x_{0}\right)=I-D \phi\left(x_{0}\right)
$$

at $x_{0}$.

\section{Proof of Theorem 1.2}

This section is devoted to the proof of Theorem 1.2 under hypotheses (H.1) and (H.2). Its proof is essentially based on three lemmas (Lemma 4.1, Lemma 4.5 and Lemma 4.6) and the unique continuation property (see [15], [33], [42]), just as in Szulkin [52, Theorem 1.3]. 


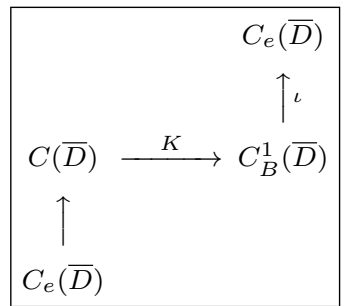

Fig. 4.1 The mapping properties of the resolvent $K$ in the spaces $C(\bar{D}), C_{e}(\bar{D})$ and $C_{B}^{1}(\bar{D})$

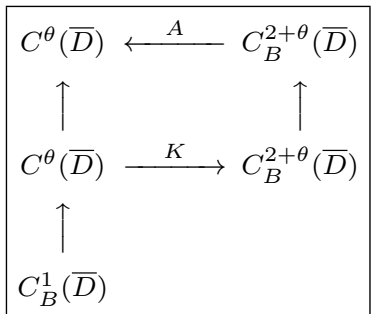

Fig. 4.2 The mapping properties of the resolvent $K$

4.1 Reduction to an operator equation

By virtue of Figure 2.1, we have the mapping properties of the resolvent $K$ in the spaces $C(\bar{D}), C_{e}(\bar{D})$ and $C_{B}^{1}(\bar{D})$ as in Figure 4.1 .

Moreover, by virtue of Theorem 2.2 we have the mapping properties of the operator $A$ and the resolvent $K$ as in Figure 4.2.

Therefore, we find that the semilinear boundary value problem

$$
\begin{cases}A u-\lambda u+f(u)=0 & \text { in } D, \\ B u=a\left(x^{\prime}\right) \frac{\partial u}{\partial \boldsymbol{\nu}}+b\left(x^{\prime}\right) u=0 & \text { on } \partial D\end{cases}
$$

is equivalent to the following operator equation:

$$
u=\lambda K u-K(f(u)) \quad \text { in } C_{e}(\bar{D}) .
$$

Now let $m(x)$ be a function in $C(\bar{D})$ such that

$$
m(x)>0 \text { on } \bar{D}
$$

and consider the following eigenvalue problem with the weight function $m(x)$ :

$$
K(m(x) u)=\mu u \quad \text { in } C_{e}(\bar{D}) .
$$

By Proposition 2.2, we find that the operator

$$
K(m(x) \cdot): C_{e}(\bar{D}) \stackrel{m(x)}{\longrightarrow} C(\bar{D}) \stackrel{K}{\longrightarrow} C_{e}(\bar{D})
$$

is strongly positive and compact (see Figure 4.3 ).

Therefore, by using the Kreln-Rutman theorem (Theorem 2.5) with $K:=$ $K(m(x) \cdot)$ we obtain the following generalization of Szulkin [52, Proposition 1.1] to the degenerate case: 


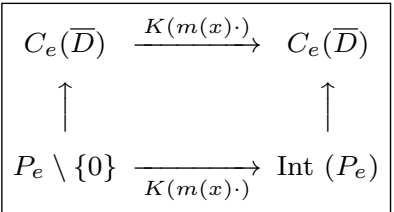

Fig. 4.3 The mapping properties of the operator $K(m(x) \cdot)$ in the ordered Banach space $C_{e}(\bar{D})$

Proposition 4.1 Assume that the weight function $m(x) \in C(\bar{D})$ satisfies condition (4.2). Then we have the following two assertions:

(i) The eigenvalue problem (4.3) has a countable number of positive eigenvalues $\mu_{j}(m)$, which may accumulate only at 0 . Hence, they may be arranged in a decreasing sequence

$$
\mu_{1}(m) \geq \mu_{2}(m) \geq \ldots,
$$

where each eigenvalue is repeated according to its multiplicity.

(ii) The largest eigenvalue $\mu_{1}(m)$ is algebraically simple, i.e., $\mu_{1}(m)>\mu_{2}(m)$, and has a positive eigenfunction. No other eigenvalues have positive eigenfunctions.

Since the operator $\mathfrak{A}$ is positive and selfadjoint in the Hilbert space $L^{2}(D)$, we can define its square root $\mathcal{C}=\mathfrak{A}^{1 / 2}$, and introduce a Hilbert space $\mathcal{H}$ as follows:

$$
\begin{aligned}
& \mathcal{H}=\text { the domain } D(\mathcal{C}) \text { with the inner product } \\
& \qquad(u, v)_{\mathcal{H}}=(\mathcal{C} u, \mathcal{C} v)_{L^{2}(D)} \text { for all } u, v \in D(\mathcal{C}) .
\end{aligned}
$$

Here it is worthwhile pointing out (see [55, Theorem 1.10]) that the explicit formula for the fractional power $\mathcal{C}=\mathfrak{A}^{1 / 2}$ on the domain $D(\mathfrak{A})$ is given by the formula

$$
\mathcal{C} u=-\frac{1}{\pi} \int_{0}^{\infty} s^{-1 / 2}(s I+\mathfrak{A})^{-1} \mathfrak{A} u d s \quad \text { for all } u \in D(\mathfrak{A}) .
$$

The next theorem gives a more concrete characterization of the Hilbert space $\mathcal{H}$ (cf. [63, Proposition 4.1]):

Theorem 4.1 The Hilbert space $\mathcal{H}=D(\mathcal{C})$ coincides with the completion of the domain

$$
D(\mathfrak{A})=\left\{u \in H^{2}(D): B u=0 \text { on } \partial D\right\}
$$

with respect to the inner product

$$
\begin{aligned}
(u, v)_{\mathcal{H}}= & (\mathfrak{A} u, v)_{L^{2}(D)} \\
= & \sum_{i, j=1}^{N} \int_{D} a^{i j}(x) \frac{\partial u}{\partial x_{i}} \frac{\partial v}{\partial x_{j}} d x+\int_{D} c(x) u \cdot v d x \\
& +\int_{\left\{a\left(x^{\prime}\right) \neq 0\right\}} \frac{b\left(x^{\prime}\right)}{a\left(x^{\prime}\right)} u \cdot v d \sigma \quad \text { for all } u, v \in D(\mathfrak{A}) .
\end{aligned}
$$

Here the last term on the right-hand side is an inner product of the Hilbert space $L^{2}(\partial D)$. 
Proof We have only to show that $D(\mathfrak{A})$ is dense in the domain $D\left(\mathfrak{A}^{1 / 2}\right)=D(\mathcal{C})$.

To do this, we remark that the operators

$$
\mathcal{C}^{-1}: L^{2}(\Omega) \longrightarrow D(\mathcal{C})
$$

and

$$
\mathcal{C}^{-1}: D\left(\mathfrak{A}^{1 / 2}\right) \longrightarrow D\left(\mathfrak{A}^{3 / 2}\right)
$$

are algebraic and topological isomorphisms, and further that

$$
D\left(\mathfrak{A}^{3 / 2}\right) \subset D(\mathfrak{A}) .
$$

Therefore, we obtain that the domain $D(\mathfrak{A})$ is dense in the domain $D(\mathcal{C})$, since $D(\mathfrak{A})$ is dense in $L^{2}(\Omega)$.

The proof of Theorem 4.1 is complete.

The situation of Theorem 4.1 can be visualized as in Figure 4.4.

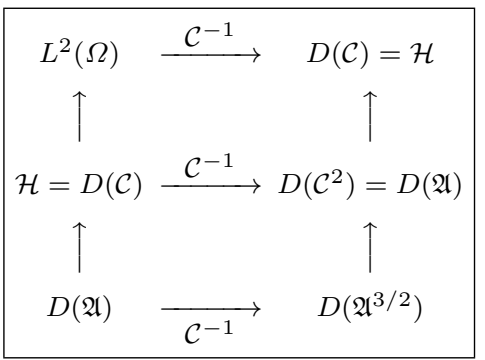

Fig. 4.4 The mapping properties of the fractional power $\mathcal{C}^{-1}=\mathfrak{A}^{-1 / 2}$

The next proposition gives the variational characterization of eigenvalues of the eigenvalue problem (4.3) (cf. [28, Proposition 1.10], [48, Chapter XIII, Theorem XIII.2], [58, Proposition 3.4]):

Proposition 4.2 Assume that the weight function $m(x) \in C(\bar{D})$ satisfies the condition (4.2). Then we have the following two assertions:

(i) The eigenvalues $\mu_{n}(m)$ are characterized as follows:

$$
\mu_{n}(m)=\sup _{F_{n}} \inf \left\{\int_{D} m(x)|u|^{2} d x:\|u\|_{\mathcal{H}}=1, u \in F_{n}\right\},
$$

where $F_{n}$ varies over all $n$-dimensional subspaces of $\mathcal{H}$.

(ii) The corresponding orthonormal eigenfunctions $\varphi_{n}(x)$ in $\mathcal{H}$ are characterized as follows:

$$
\begin{aligned}
\left(\varphi_{n}, v\right)_{\mathcal{H}}= & \sum_{i, j=1}^{N} \int_{D} a^{i j}(x) \frac{\partial \varphi_{n}}{\partial x_{i}} \frac{\partial v}{\partial x_{j}} d x+\int_{D} c(x) \varphi_{n} \cdot v d x \\
& +\int_{\left\{a\left(x^{\prime}\right) \neq 0\right\}} \frac{b\left(x^{\prime}\right)}{a\left(x^{\prime}\right)} \varphi_{n} \cdot v d \sigma
\end{aligned}
$$




$$
\begin{aligned}
& =\frac{1}{\mu_{n}(m)} \int_{D} m(x) \varphi_{n} \cdot v d x \quad \text { for all } v \in \mathcal{H} . \\
\mu_{n}(m) & =\int_{D} m(x)\left|\varphi_{n}\right|^{2} d x .
\end{aligned}
$$

(iii) If $m_{1}(x) \leq m_{2}(x)$ for all $x \in \bar{D}$, then we have $\mu_{n}\left(m_{1}\right) \leq \mu_{n}\left(m_{2}\right)$ for all $n$. If $m_{1}(x)<m_{2}(x)$ for all $x \in \bar{D}$, then we have $\mu_{n}\left(m_{1}\right)<\mu_{n}\left(m_{2}\right)$ for all $n$.

Part (iii) is an immediate consequence of the maxmini formula of eigenvalues $\mu_{n}(m)$ given by part (i) (see [58, Proposition 3.5]).

\subsection{Proof of Theorem 1.2}

The proof of Theorem 1.2 is divided into five steps.

Step 1: In order to study globally the bifurcation solution curves, we need the following generalization of Szulkin [52, Lemma 2.1] to the degenerate case:

Lemma 4.1 If $u$ is a positive or negative solution of the nonlinear operator equation (4.1) with $\lambda_{1}<\lambda<\infty$, then $u$ is a regular point of the mapping

$$
G(\lambda, u): \mathbf{R} \times C(\bar{D}) \longrightarrow C(\bar{D}),
$$

given by the formulas

$$
\begin{aligned}
& G(\lambda, u):=u-F(\lambda, u), \\
& F(\lambda, u):=K(\lambda u-f(u)) .
\end{aligned}
$$

Namely, the partial Fréchet derivative $G_{u}(\lambda, u)$ at $u$ is invertible.

Proof First, we remark that the Fréchet derivative of $G(\lambda, \cdot)$ at $u$ is equal to the following:

$$
G_{u}(\lambda, u)=I-F_{u}(\lambda, u)=I-K\left(\lambda-f^{\prime}(u)\right) .
$$

Since the mapping

$$
F(\lambda, u)=\lambda K u-K(f(u))
$$

is compact, it suffices to show that the operator equation

$$
v=F_{u}(\lambda, u) v=K\left(\lambda v-f^{\prime}(u) v\right)
$$

has only the trivial solution $v=0$.

Our proof is based on a reduction to absurdity. Assume, to the contrary, that the operator equation (4.5) has a nontrivial solution $u$. Then the eigenvalue problem with weight $\lambda-f^{\prime}(u)$

$$
K\left(\lambda-f^{\prime}(u)\right) v=\mu v \quad \text { in } C(\bar{D})
$$

has an eigenvalue 1 . This implies that

$$
\mu_{k}\left(\lambda-f^{\prime}(u)\right)=1 \quad \text { for some } k \geq 1 .
$$

If $u$ is a positive solution of the equation

$$
F(\lambda, u)=K(\lambda u-f(u))=u \quad \text { in } C(\bar{D}),
$$


then the equation can be written in the form

$$
u=K\left(\lambda-\frac{f(u)}{u}\right) u \quad \text { in } C(\bar{D}),
$$

where

since we have the formula

$$
\frac{f(t)}{t}=0 \quad \text { for } t=0
$$

$$
\lim _{t \rightarrow 0} \frac{f(t)}{t}=0
$$

Hence the eigenvalue problem with weight $\lambda-f(u) / u$

$$
K\left(\lambda-\frac{f(u)}{u}\right) v=\mu v \quad \text { in } C(\bar{D})
$$

has an eigenvalue 1 . This implies that

$$
\mu_{j}\left(\lambda-\frac{f(u)}{u}\right)=1 \quad \text { for some } j \geq 1
$$

with a corresponding positive eigenfunction $u$.

However, it follows from condition (1.4) and the properties of $f(t)$ that

$$
\lambda-\frac{f(t)}{t}>\lambda-f^{\prime}(t)>\lambda_{1}-f^{\prime}(t)>0 \quad \text { for all } t \neq 0 .
$$

Therefore, by applying part (ii) of Proposition 4.1 with (see formula (4.4))

$$
m(x):=\lambda-\frac{f(u(x))}{u(x)}
$$

we obtain that $j=1$, that is,

$$
\mu_{1}\left(\lambda-\frac{f(u)}{u}\right)=1
$$

Moreover, it follows from an application of Lemma 2.2 with $v:=\lambda u-f(u)$ that the solution

$$
u=K(\lambda u-f(u))
$$

satisfies the condition

$$
u(x)>0 \text { in } D .
$$

By combining inequality (4.8) and Proposition 4.2, we obtain from formulas (4.6) and (4.9) that

$$
1=\mu_{1}\left(\lambda-\frac{f(u)}{u}\right)>\mu_{1}\left(\lambda-f^{\prime}(u)\right) \geq \mu_{k}\left(\lambda-f^{\prime}(u)\right)=1 .
$$

This is a contradiction.

Summing up, we have proved that the operator equation (4.5) has only the trivial solution $v=0$. In view of the Fredholm alternative, this implies that the partial Fréchet derivative $G_{u}(\lambda, u)$ at $u$ is bijective and hence invertible.

Similarly, we can prove that a negative solution $u$ of the nonlinear operator equation (4.1) is a regular point of $G(\lambda, u)$.

The proof of Lemma 4.1 is complete. 
Step 2: Secondly, we show that the positive solution of the nonlinear operator equation (4.1) is unique. The proof is essentially based on the following uniqueness theorem for order convex maps (cf. [5, Theorem 24.2]):

Theorem 4.2 (the uniqueness theorem) Let $(E, Q)$ be an ordered Banach space having the positive cone $Q$ with nonempty interior. If $\sigma$ is a positive number, we let

$$
\bar{Q}_{\sigma}=\{u \in Q:\|u\| \leq \sigma\} .
$$

Assume that a mapping

$$
f: \bar{Q}_{\sigma} \longrightarrow E
$$

satisfies the following two conditions:

(A) $f$ is strongly increasing, that is, if $u, v \in \bar{Q}_{\sigma}$ and if $u \leq v$ and $v \neq u$, then it follows that $f(v)-f(u)$ is an interior point of $Q$.

(B) $f$ is strongly sublinear, that is, $f(0) \geq 0$ and if $u \in \bar{Q}_{\sigma}$ and $u \neq 0$, then it follows that $f(\tau u)-\tau f(u)$ is an interior point of $Q$ for every $0<\tau<1$.

Then the mapping $f$ has at most one positive fixed point.

Proof Our proof is based on a reduction to absurdity. Assume, to the contrary, that $u_{0}$ and $u_{1}$ are two positive fixed points of $f$ :

$$
\left\{\begin{array}{l}
u_{0} \in \bar{P}_{\sigma}, f\left(u_{0}\right)=u_{0}, \\
u_{1} \in \bar{P}_{\sigma}, f\left(u_{1}\right)=u_{1} .
\end{array}\right.
$$

Without loss of generality, we may assume that

$$
u_{1} \not \leq u_{0} \text {. }
$$

Since $f$ is strongly monotone increasing, it follows that

$$
u_{0}=f\left(u_{0}\right) \gg f(0) \geq 0 .
$$

This implies that

$$
u_{0} \in \operatorname{Int}(P) .
$$

Moreover, we can find a positive number $\tau$ such that

$$
u_{0}-\tau u_{1} \in \partial(P)
$$

Here we remark that $0<\tau<1$. Indeed, it suffices to note that $u_{1} \not \leq u_{0}$.

However, since $f$ is strongly sublinear and strongly monotone increasing, we obtain that

$$
u_{0}=f\left(u_{0}\right) \geq f\left(\tau u_{1}\right) \gg \tau f\left(u_{1}\right)=\tau u_{1},
$$

so that

$$
u_{0}-\tau u_{1} \in \operatorname{Int}(P) .
$$

This is a contradiction.

The proof of Theorem 4.2 is complete. 
In the proof of Theorem 1.2, we shall apply Theorem 4.2 with

$$
\begin{aligned}
& E:=C_{e}(\bar{D}), \\
& Q:=P_{e}=\left\{u \in C_{e}(\bar{D}): u \geq 0 \text { on } \bar{D}\right\}, \\
& f:=\left.F(\lambda, \cdot)\right|_{P_{e}}=\left.K(\lambda \cdot-f(\cdot))\right|_{P_{e}} .
\end{aligned}
$$

If $\sigma$ is a positive number, we let

$$
\left(\overline{P_{e}}\right)_{\sigma}:=\left\{u \in P_{e}:\|u\| \leq \sigma \text { on } \bar{D}\right\} .
$$

We have only to consider in the space $\left(\overline{P_{e}}\right)_{\sigma}$ for every $\sigma>0$. Indeed, if $u_{1}$ and $u_{2}$ are two positive solutions of the nonlinear operator equation (4.1), then we can find a constant $\sigma>0$ such that $\left\|u_{1}\right\|,\left\|u_{2}\right\| \leq \sigma$ on $\bar{D}$, so that $u_{1}, u_{2} \in\left(\overline{P_{e}}\right)_{\sigma}$.

Then we have the following lemma:

Lemma 4.2 The operator $F(\lambda, \cdot)$ maps $\left(\overline{P_{e}}\right)_{\sigma}$ into $P_{e}$.

Proof Let $u$ be an arbitrary function in $\left(\overline{P_{e}}\right)_{\sigma}$. By applying inequality (1.4), we find that

$$
\lambda u-f(u) \in P .
$$

Hence it follows from an application of Proposition 2.2 that

$$
F(\lambda, u)=K(\lambda u-f(u)) \in P_{e} .
$$

The proof of Lemma 4.2 is complete.

Moreover, we have the following lemma:

Lemma 4.3 The operator $F(\lambda, \cdot):\left(\overline{P_{e}}\right)_{\sigma} \longrightarrow P_{e}$ is strongly increasing.

Proof The proof of Lemma 4.3 is based on the following claim:

Claim 4.1 The operator $F(\lambda, \cdot):[\phi, \psi] \rightarrow C(\bar{D})$ is increasing. Here $[\phi, \psi]$ is the order interval defined by the formula

$$
[\phi, \psi]=\{u \in C(\bar{D}): \phi \leq u \leq \psi \text { on } \bar{D}\} .
$$

Proof Let $u$ and $v$ be arbitrary functions in $C(\bar{D})$ satisfying $\phi \leq u \leq v \leq \psi$ on $\bar{D}$. Then we have the assertions

$$
\begin{aligned}
& (F(\lambda, v(x))-F(\lambda, u(x))) \\
= & \begin{cases}0 & \text { if } v(x)=u(x), \\
\left(\frac{F(\lambda, v(x))-F(\lambda, u(x))}{v(x)-u(x)}\right)(v(x)-u(x)) & \text { if } v(x)>u(x) .\end{cases}
\end{aligned}
$$

Hence it follows from inequality (1.4) and Lemma 2.2 that

$$
F(\lambda, v)-F(\lambda, u)=K(\lambda(v-u)-(f(v)-f(u))) \geq 0 \quad \text { on } \bar{D},
$$

or equivalently

$$
F(\lambda, u) \leq F(\lambda, v) \quad \text { on } \bar{D} .
$$

This proves that $F(\lambda, \cdot)$ is increasing.

The proof of Claim 4.1 is complete. 
Therefore, Lemma 4.3 follows by combining Claim 4.1 and Proposition 2.2.

Lemma 4.4 The operator $F(\lambda, \cdot):\left(\overline{P_{e}}\right)_{\sigma} \longrightarrow P_{e}$ is strongly sublinear.

Proof Let $u$ be an arbitrary function in $\left(\overline{P_{e}}\right)_{\sigma}$ but $u \neq 0$. Since $f(t)$ is convex for $t>0$, we have, for every $0<\tau<1$,

$$
\begin{cases}f(\tau u(x))>\tau f(u(x)) & \text { if } u(x)>0 \\ f(\tau u(x))=f(0)=0 & \text { if } u(x)=0\end{cases}
$$

and so

$$
\begin{aligned}
& F(\tau \lambda, u)-\tau F(\lambda, u) \\
= & K(\tau \lambda u-f(\tau u))-\tau(\lambda u-f(u)) \geq 0 \text { and } \not \equiv 0 \text { on } \bar{D} .
\end{aligned}
$$

Hence it follows from an application of Proposition 2.2 that the function

$$
F(\tau \lambda, u)-\tau F(\lambda, u)
$$

is an interior point of $P_{e}$ for every $0<\tau<1$.

The proof of Lemma 4.4 is complete.

By combining Lemmas 4.2, 4.3 and 4.4, we have proved that the mapping

$$
F(\lambda, \cdot):\left(\overline{P_{e}}\right)_{\sigma} \longrightarrow P_{e}
$$

satisfies conditions (A) and (B) of Theorem 4.2 with $E:=C_{e}(\bar{D})$ and $Q:=P_{e}$.

Therefore, the uniqueness of positive fixed points of the nonlinear operator equation (4.1) follows from an application of Theorem 4.2.

Step 3: The next lemma is a generalization of Szulkin [52, Lemma 2.2] to the degenerate case, and is an essential step in the proof of Theorem 1.2:

Lemma 4.5 The nonlinear operator equation (4.1) has a unique positive solution for each $\lambda_{1}<\lambda<\lambda_{1}+k_{+}$. No positive solutions exist for $\lambda \geq \lambda_{1}+k_{+}$. The uniform norm $\left\|u_{+}(\lambda)\right\|$ of the positive solution $u_{+}(\lambda)$ tends to $\infty$ as $\lambda \rightarrow \lambda_{1}+k_{+}$:

$$
\lim _{\lambda \rightarrow \lambda_{1}+k_{+}}\left\|u_{+}(\lambda)\right\|=\infty .
$$

Similar assertions hold true for negative solutions $u_{-}(\lambda)$ of the nonlinear operator equation (4.1) for each $\lambda_{1}<\lambda<\lambda_{1}+k_{-}$.

Proof The proof of Lemma 4.5 is divided into three steps.

Step (i): First, we show that the nonlinear operator equation (4.1) has a positive solution.

By inequality (4.8), it follows that the mapping

$$
\begin{aligned}
F(\lambda, \cdot)=K(\lambda \cdot-f(\cdot)): C(\bar{D}) & \longrightarrow C(\bar{D}) \\
u & \longmapsto K(\lambda u-f(u))
\end{aligned}
$$

maps the positive cone $P$ into itself. Moreover, we remark that $F(\lambda, \cdot)$ is right asymptotically linear. Indeed, we have the assertion

$$
\frac{F(\lambda, u)}{u}=\lambda K-K\left(\frac{f(u)}{u}\right) \rightarrow\left(\lambda-k_{+}\right) K \quad \text { as } u \rightarrow+\infty
$$


and so

$$
F_{+}^{\prime}(\lambda, \infty) v=\left(\lambda-k_{+}\right) K v \quad \text { for all } v \in P .
$$

Here $F_{+}^{\prime}(\lambda, \cdot)$ denotes the right Fréchet derivative of $F(\lambda, \cdot)$.

Similarly, it follows that $F(\lambda, \cdot)$ is right differentiable at zero, since we have the formula

$$
\frac{F(\lambda, u)}{u}=\lambda K-K\left(\frac{f(u)}{u}\right) \rightarrow \lambda K \quad \text { as } u \rightarrow 0
$$

and so

$$
F_{+}^{\prime}(\lambda, 0) v=\lambda K v \quad \text { for all } v \in P
$$

The proof of Lemma 4.5 is based on the following positive fixed point theorem for differentiable maps ([5, Theorem 13.6]):

Theorem 4.3 (Amann) Let $f: P \rightarrow P$ be a completely continuous map such that $f(0)=0$. Assume that $f$ is asymptotically linear and right differentiable at zero such that 1 is not an eigenvalue of $f^{\prime}(\infty)$ or of $f_{+}^{\prime}(0)$ to a positive eigenvector. Then $f$ has at least one positive fixed point, provided that one of the following two conditions (I) and (II) is satisfied:

(I) $f_{+}^{\prime}(0)$ has no positive eigenvector to an eigenvalue greater than one, whereas $f^{\prime}(\infty)$ possesses such a positive eigenvector.

(II) $f_{+}^{\prime}(0)$ possesses a positive eigenvector to an eigenvalue greater than one, but this is not the case for $f^{\prime}(\infty)$.

If $\lambda_{1}<\lambda<\lambda_{1}+k_{+}$, we find that

$$
\begin{aligned}
& \left\{\begin{array}{l}
\left(\lambda-k_{+}\right) K v=F_{+}^{\prime}(\lambda, \infty) v=\mu v, \\
v \in P \backslash\{0\}
\end{array}\right. \\
& \Longleftrightarrow \begin{array}{l}
K v=\left(\frac{\mu}{\lambda-k_{+}}\right) v, \quad \Longleftrightarrow \frac{\lambda-k_{+}}{\mu}=\lambda_{1} \\
v \in P \backslash\{0\}
\end{array} \\
& \Longleftrightarrow \begin{array}{l}
\mu \frac{\lambda-k_{+}}{\lambda_{1}}<1 \quad \text { for } \lambda<\lambda_{1}+k_{+} .
\end{array}
\end{aligned}
$$

This proves that $F_{+}^{\prime}(\lambda, \infty)$ possesses a positive eigenvector to an eigenvalue $\mu<1$.

On the other hand, we find that

$$
\begin{aligned}
& \quad\left\{\begin{array}{l}
\lambda K v=F_{+}^{\prime}(\lambda, 0) v=\nu v, \\
v \in P \backslash\{0\}
\end{array}\right. \\
& \Longleftrightarrow\left\{\begin{array}{l}
K v=\frac{\nu}{\lambda} v, \\
v \in P \backslash\{0\}
\end{array}\right. \\
& \Longleftrightarrow \quad \Longleftrightarrow \frac{\lambda}{\nu}=\lambda_{1}
\end{aligned}
$$

This proves that $F_{+}^{\prime}(, \lambda)$ possesses a positive eigenvector to an eigenvalue $\nu>1$.

Summing up, we have proved that if $\lambda_{1}<\lambda<\lambda_{1}+k_{+}$, then it follows that: 
(II) $F_{+}^{\prime}(\lambda, \infty)=\left(\lambda-k_{+}\right) K$ possesses a positive eigenvector to an eigenvalue $\mu<1$, while $F_{+}^{\prime}(\lambda, 0)=\lambda K$ possesses a positive eigenvector to an eigenvalue $\nu>1$.

Therefore, by applying Theorem 4.3 we obtain that the map $F(\lambda, \cdot)$ has a positive fixed point $u$ :

$$
F(\lambda, u)=K(\lambda u-f(u))=u, \quad u \in P .
$$

Step (ii): Secondly, we show that the nonlinear operator equation (4.1) has no positive solutions for $\lambda \geq \lambda_{1}+k_{+}$.

Our proof is based on a reduction to absurdity. Assume, to the contrary, that $u$ is a positive solution of the nonlinear operator equation (4.1) for $\lambda \geq \lambda_{1}+k_{+}$. Then we have the assertion

$$
K\left(\lambda-\frac{f(u)}{u}\right) u=K(\lambda u-f(u))=u \quad \text { in } C(\bar{D}) .
$$

This implies that

$$
\mu_{1}\left(\lambda-\frac{f(u)}{u}\right)=1
$$

with a corresponding positive eigenfunction $u$.

On the other hand, since we have, by inequality (1.4),

$$
\lambda-f^{\prime}(u)>\lambda-\sup _{t>0} f^{\prime}(t)=\lambda-k_{+},
$$

we find from the proof of Proposition 4.2 that

$$
\begin{aligned}
1 & =\mu_{1}\left(\lambda-\frac{f(u)}{u}\right)>\mu_{1}\left(\lambda-f^{\prime}(u)\right)>\mu_{1}\left(\lambda-k_{+}\right) \\
& \geq \mu_{1}\left(\lambda_{1}\right)=1 \quad \text { for } \lambda \geq \lambda_{1}+k_{+} .
\end{aligned}
$$

This is a contradiction.

Step (iii): Thirdly, in order to show that the norm of the positive solution tends to $\infty$ as $\lambda \rightarrow \lambda_{1}+k_{+}$we use the compactness of the resolvent $K$.

Our proof is based on a reduction to absurdity. Assume, to the contrary, that there exist a sequence $\left\{\left(u_{n}, \lambda^{(n)}\right)\right\}$ and a constant $C>0$ such that

$$
\begin{cases}K\left(\lambda^{(n)} u_{n}-f\left(u_{n}\right)\right)=F\left(\lambda^{(n)}, u_{n}\right)=u_{n} & \text { for all } n, \\ \left\|u_{n}\right\| \leq C & \text { for all } n, \\ \lambda^{(n)} \longrightarrow \lambda_{1}+k_{+} & \text {as } n \rightarrow \infty .\end{cases}
$$

By virtue of the compactness of $K$, (by passing to a subsequence) we may assume that

$$
u_{n}=F\left(u_{n}, \lambda^{(n)}\right) \longrightarrow u_{0} \quad \text { in } C(\bar{D}) .
$$

Then we have the formula

$$
u_{0}=F\left(\lambda_{1}+k_{+}, u_{0}\right) \quad \text { in } C(\bar{D}) .
$$

This implies that $u_{0}$ is a positive solution of the nonlinear operator equation (4.1) for $\lambda=\lambda_{1}+k_{+}$. 
However, it follows from Step 2 (the uniqueness of positive fixed points of the nonlinear operator equation (4.1)) that

$$
u_{0}=0
$$

so that

$$
u_{n} \longrightarrow 0 \quad \text { in } C(\bar{D}) \text {. }
$$

Since $f^{\prime}(0)=0$, we obtain from assertions (4.10) and (4.11) that

$$
\begin{aligned}
1 & =\mu_{1}\left(\lambda^{(n)}-\frac{f\left(u_{n}\right)}{u_{n}}\right)>\mu_{1}\left(\lambda^{(n)}-f^{\prime}\left(u_{n}\right)\right) \\
& >\mu_{1}\left(\lambda_{1}\right)=1 \text { for } n \text { sufficiently large. }
\end{aligned}
$$

This is a contradiction.

In order to prove the lemma for negative solutions, we repeat the above arguments in the "positive" cone $\{u \in C(\bar{D}): u \leq 0\}$.

The proof of Lemma 4.5 is complete.

Step 4: The next lemma is a generalization of Szulkin [52, Lemma 2.3] to the degenerate case, which asserts that the nonlinear operator equation (4.1) has no nontrivial solutions for $\lambda_{1}-\delta \leq \lambda \leq \lambda_{1}$ :

Lemma 4.6 Let $\delta$ be the constant as in condition (1.4):

$$
\sup _{t \in \mathbf{R}}\left|f^{\prime}(t)\right|<\lambda_{1}-\delta \quad \text { for some constant } 0<\delta<\lambda_{1} .
$$

Then the nonlinear operator equation (4.1) has no nontrivial solutions for $\lambda_{1}-\delta \leq$ $\lambda \leq \lambda_{1}$.

Proof Our proof is based on a reduction to absurdity. Assume, to the contrary, that $u$ is a nontrivial solution of the nonlinear operator equation (4.1)

$$
K(\lambda u-f(u))=u \quad \text { in } C(\bar{D}) .
$$

Then the eigenvalue problem with weight

$$
K\left(\lambda-\frac{f(u)}{u}\right) v=\mu v \quad \text { in } C(\bar{D})
$$

has an eigenvalue 1 , that is,

$$
\mu_{k}\left(\lambda-\frac{f(u)}{u}\right)=1 \quad \text { for some } k \geq 1,
$$

with a corresponding eigenfunction $u$. Since $f(0)=f^{\prime}(0)=0$ and $f(t)$ is convex for $t>0$ and is concave for $t<0$, it follows that

$$
\lambda-\frac{f(u(x))}{u(x)} \leq \lambda \quad \text { for } u(x) \neq 0 .
$$

This implies that

$$
1=\mu_{k}\left(\lambda-\frac{f(u)}{u}\right) \leq \mu_{k}(\lambda) \leq \frac{\lambda}{\lambda_{k}} \quad \text { for } \lambda_{1}-\delta \leq \lambda \leq \lambda_{1} .
$$


Hence, we have the assertions

$$
k=1, \quad \lambda=\lambda_{1},
$$

and the corresponding eigenfunction $u(x)$ is of constant sign:

$$
u(x) \geq 0 \text { or } u(x) \leq 0 \text { in } D .
$$

Moreover, it follows from Lemma 2.2 that

$$
u(x)>0 \text { or } u(x)<0 \text { in } D .
$$

Therefore, since we have the inequality

$$
\lambda_{1}-\frac{f(u(x))}{u(x)}>\lambda_{1} \quad \text { for } u(x) \neq 0,
$$

we find from formula (4.12) with $k:=1$ and $\lambda:=\lambda_{1}$ that

$$
1=\mu_{1}\left(\lambda_{1}-\frac{f(u)}{u}\right)<\mu_{1}\left(\lambda_{1}\right)=1 .
$$

This is a contradiction.

The proof of Lemma 4.6 is complete.

Remark 4.1 By the argument of Plastock [46, p. 321], we can prove that the mapping $G(\lambda, \cdot)$ defined in Lemma 4.1 is a homeomorphism of $C(\bar{D})$ onto itself for any $\lambda \leq \lambda_{1}$.

Step 5: Finally, we show that each nontrivial solution of the nonlinear operator equation (4.1) for $\lambda_{1}<\lambda \leq \lambda_{2}$ must necessarily be positive or negative in $D$.

If $u \neq 0$ is a solution, then it follows that

$$
\mu_{k}\left(\lambda-\frac{f(u)}{u}\right)=1 \quad \text { for some } k \geq 1,
$$

with a corresponding eigenfunction $u$, just as in the proof of Lemma 4.6.

However, by the unique continuation property (see [15], [33], [42]) it follows that

$$
u(x) \neq 0 \quad \text { almost everywhere in } D .
$$

This implies that

$$
\lambda-\frac{f(u(x))}{u(x)}<\lambda \quad \text { almost everywhere in } D .
$$

Hence we have the assertion

$$
\mu_{2}\left(\lambda-\frac{f(u)}{u}\right) \leq \mu_{2}(\lambda)=\frac{\lambda}{\lambda_{2}} \leq 1 \quad \text { for } \lambda_{1}<\lambda \leq \lambda_{2} .
$$

Since the first eigenvalue $\mu_{1}\left(\lambda-\frac{f(u)}{u}\right)$ is algebraically simple, it follows that

$$
\mu_{1}\left(\lambda-\frac{f(u)}{u}\right)=1 \quad \text { for } \lambda_{1}<\lambda \leq \lambda_{2},
$$

with a corresponding eigenfunction $u$. 
Therefore, we have proved that each nontrivial solution of the operator equation

$$
K\left(\lambda-\frac{f(u)}{u}\right) v=v \quad \text { in } C(\bar{D})
$$

is of constant sign. Since $u$ is a solution of this equation, it follows that $u$ is positive or negative in $D$.

Now the proof of Theorem 1.2 is complete.

\section{The Lyapunov-Schmidt procedure in the Banach space $C(\bar{D})$}

In the proof of Theorems 1.3 and 1.4 we make use of the Lyapunov-Schmidt procedure ([40], [50]) in order to study the nonlinear operator equation (4.1), which reduces an infinite-dimensional problem to a finite-dimensional system (Theorem 5.1). This section is the heart of the subject.

We assume that hypotheses (H.1) and (H.2) are satisfied. Let $e_{1}(x)$ be the normalized positive solution of the operator equation

$$
e_{1}-\lambda_{1} K e_{1}=0 \quad \text { in } C(\bar{D}),
$$

or equivalently (see Theorem 1.1),

$$
\begin{cases}A e_{1}=\lambda_{1} e_{1} & \text { in } D, \\ B e_{1}=0 & \text { on } \partial D, \\ \left\|e_{1}\right\|_{L^{2}(D)}=1 . & \end{cases}
$$

First, we have the following orthogonal decomposition in the Hilbert space $L^{2}(D)$

$L^{2}(D)$

$=N\left(I-\lambda_{1} K\right) \oplus R\left(I-\lambda_{1} K\right)$

$=\left\{u \in L^{2}(D):\left(A-\lambda_{1}\right) u=0, B u=0\right\} \oplus\left\{\left(I-\lambda_{1} K\right) u: u \in H^{2}(D), B u=0\right\}$.

$=\operatorname{span}\left[e_{1}\right] \oplus R\left(I-\lambda_{1} K\right)$.

Indeed, it suffices to note that the operator $\mathfrak{A}$ is selfadjoint in $L^{2}(D)$ and that

$$
K=\mathfrak{A}^{-1} .
$$

Hence, by restricting the orthogonal decomposition (5.3) to the subspace $L^{p}(D)$ for $N<p<\infty$ we obtain the orthogonal decomposition

$$
L^{p}(D)=\operatorname{span}\left[e_{1}\right] \oplus\left(R\left(I-\lambda_{1} K\right) \cap L^{p}(D)\right) \text { for } N<p<\infty .
$$

However, it follows from an application of the regularity theorem ([65, Theorem 7.1]) that

$$
R\left(I-\lambda_{1} K\right) \cap L^{p}(D)=\left\{\left(I-\lambda_{1} K\right) v: v \in C(\bar{D})\right\} .
$$

Indeed, it suffices to note the following:

$$
u-\lambda_{1} K u \in C(\bar{D}), u \in L^{p}(D)
$$




$$
\begin{aligned}
& \Longrightarrow \\
& \quad K u \in W^{2, p}(D) \subset C^{2-N / p}(\bar{D}) \text { for } N<p<\infty \\
& \Longrightarrow \\
& u=\left(u-\lambda_{1} K u\right)+\lambda_{1} K u \in C(\bar{D}) .
\end{aligned}
$$

Hence, by restricting the orthogonal decomposition (5.4) to the subspace $C(\bar{D})$ of $L^{2}(D)$ we obtain the orthogonal decomposition (see Figure 5.1)

$$
C(\bar{D})=\operatorname{span}\left[e_{1}\right] \oplus\left\{\left(I-\lambda_{1} K\right) v: v \in C(\bar{D})\right\}=\operatorname{span}\left[e_{1}\right] \oplus W,
$$

where

$$
W:=\left\{\left(I-\lambda_{1} K\right) v: u \in C(\bar{D})\right\}
$$

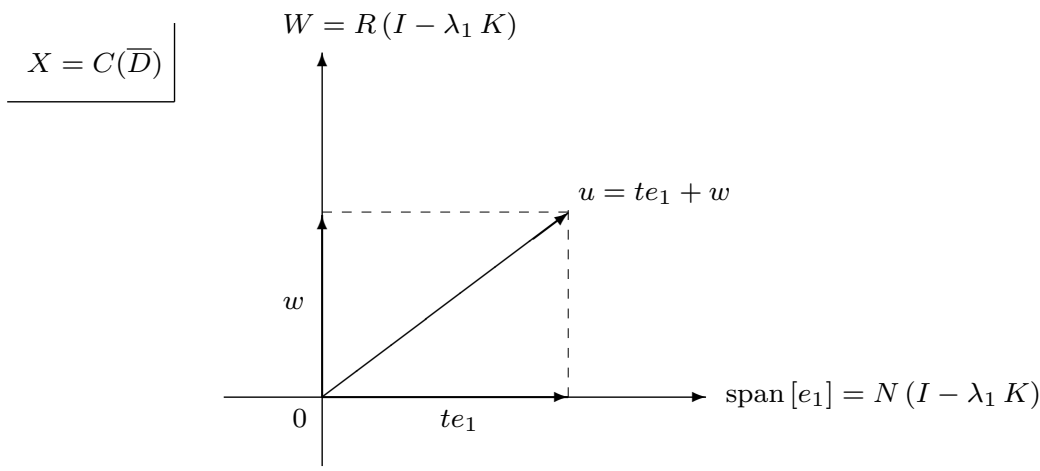

Fig. 5.1 The orthogonal decomposition (5.5) of $X=C(\bar{D})$

If we define the orthogonal projection $Q$ from $C(\bar{D})$ onto $W$ along $N\left(I-\lambda_{1} K\right)$ by the formula

$$
Q u=u-\left(\int_{D} w(x) e_{1}(x) d x\right) e_{1} \quad \text { for all } u \in C(\bar{D})
$$

or equivalently,

$$
(I-Q) u=\left(\int_{D} w(x) e_{1}(x) d x\right) e_{1} \quad \text { for all } u \in C(\bar{D}),
$$

then it is easy to see that

$$
W=Q(X)=\left\{w \in C(\bar{D}): \int_{D} w(x) e_{1}(x) d x=0\right\} .
$$

Moreover, let $e_{1}^{*}$ be a continuous linear functional on the space $C(\bar{D})$ such that

$$
\left\{\begin{array}{l}
\left\langle e_{1}, e_{1}^{*}\right\rangle=1, \\
\left\langle w, e_{1}^{*}\right\rangle=0 \text { for all } w \in W .
\end{array}\right.
$$


Then it follows from the orthogonal decomposition (5.5) that every function $u \in$ $C(\bar{D})$ can be written uniquely in the form (see Figure 5.1)

$$
u=t e_{1}+w \text { for } t=\left\langle u, e_{1}^{*}\right\rangle \text { and } w \in W .
$$

Hence we have the assertions

$$
\begin{aligned}
& \begin{cases}A u-\lambda u+f(u)=0 & \text { in } D, \\
B u=0 & \text { in } \partial D\end{cases} \\
& \Longleftrightarrow \\
& u-\lambda K u+K(f(u))=0 \quad \text { in } C_{e}(\bar{D}) \\
& \Longleftrightarrow \\
& \left\{\begin{array}{l}
u=t e_{1}+w, \\
w-\lambda K w+Q\left(K\left(f\left(t e_{1}+w\right)\right)\right)=0 \quad \text { in } C(\bar{D}), \\
\left\langle t\left(1-\frac{\lambda}{\lambda_{1}}\right) e_{1}+(I-Q) K\left(f\left(t e_{1}+w\right)\right), e_{1}^{*}\right\rangle=0 .
\end{array}\right.
\end{aligned}
$$

Summing up, we are reduced to the infinite-dimensional equation

$$
w-\lambda K w+Q\left(K\left(f\left(t e_{1}+w\right)\right)\right)=0 \quad \text { in } C(\bar{D})
$$

and the one-dimensional equation

$$
\Psi(t, w):=t\left(1-\frac{\lambda}{\lambda_{1}}\right)+\left\langle K\left(f\left(t e_{1}+w\right)\right), e_{1}^{*}\right\rangle=0 .
$$

However, the Fréchet derivative of the left-hand side of the infinite-dimensional equation (5.8) at $t=0$ and $\lambda=\lambda_{1}$ is given by the formula

$$
I-\lambda_{1} K=\left(\begin{array}{cc}
\left.\left(I-\lambda_{1} K\right)\right|_{W} & 0 \\
0 & 1 / \lambda_{1}
\end{array}\right): \begin{gathered}
W \\
\operatorname{span}\left[e_{1}\right]
\end{gathered} \begin{gathered}
W \\
\oplus \\
\operatorname{span}\left[e_{1}\right]
\end{gathered}
$$

Since we have, by formula $(5.5)$,

$$
C(\bar{D})=W \oplus \operatorname{span}\left[e_{1}\right],
$$

we find from formula (5.10) and Figure 5.1 that the Fréchet derivative $I-\lambda_{1} K$ of the infinite-dimensional equation (5.8) at $t=0$ and $\lambda=\lambda_{1}$ is an isomorphism of the space $C(\bar{D})$.

Therefore, it follows from an application of the implicit function theorem (Theorem 3.1) that there exists a constant $\varepsilon_{1}>0$ such that if $|t|<\varepsilon_{1}$ and $\left|\lambda-\lambda_{1}\right|<\varepsilon_{1}$, then the infinite-dimensional equation (5.8) has a unique solution $w(t, \lambda)$ :

$$
\begin{aligned}
& w(t, \lambda)-\lambda K w(t, \lambda)+Q\left(K\left(f\left(t e_{1}+w(t, \lambda)\right)\right)\right)=0 \text { in } C(\bar{D}), \\
& \quad \text { for }|t|<\varepsilon_{1} \text { and }\left|\lambda-\lambda_{1}\right|<\varepsilon_{1} .
\end{aligned}
$$

If we take $\lambda \in\left(\lambda_{1}, \lambda_{1}+\varepsilon_{1}\right)$, then we find that all small solutions $u=t e_{1}+$ $w(t, \lambda)$ of the nonlinear operator equation (4.1) are in one-to-one correspondence with the solutions $t$ of the one-dimensional equation

$$
\Phi(t, w(t, \lambda)):=t\left(1-\frac{\lambda}{\lambda_{1}}\right)+\left\langle K\left(f\left(t e_{1}+w(t, \lambda)\right)\right), e_{1}^{*}\right\rangle=0 \quad \text { for }|t|<\varepsilon_{1} .
$$

By combining formulas (5.7), (5.11) and (5.12), we have proved the following theorem: 
Theorem 5.1 There exists a constant $\varepsilon_{1}>0$ such that every small solution $u$ of the semilinear problem (1.2) can be uniquely expressed in the form

$$
u=t e_{1}+w=t e_{1}+w(t, \lambda) \quad \text { for }|t|<\varepsilon_{1} \text { and } \lambda_{1}<\lambda<\lambda_{1}+\varepsilon_{1},
$$

where the $w(t, \lambda)$ satisfy the infinite-dimensional equation (5.11) and the $t$ satisfy the one-dimensional equation (5.12).

\section{Proof of Theorem 1.3}

In this section we study the semilinear elliptic boundary value problem (1.2) under the condition that $f(t)$ is bounded on $\mathbf{R}$. It turns out that the set of nontrivial solutions has properties considerably different from Theorem 1.2.

The semilinear elliptic boundary value problem (1.2) may be converted into the nonlinear operator equation (4.1) which we rewrite as follows:

$$
G(\lambda, u):=u-\lambda K u+K(f(u))=0 \quad \text { in } C(\bar{D}) .
$$

The purpose of this section is to prove the following generalization of Szulkin [52, Theorem 5.1] to the degenerate case:

Theorem 6.1 Assume that hypotheses (H.1) and (H.2) are satisfied and let $f(t)$ be a bounded, $C^{1}$ function on $\mathbf{R}$ such that

$$
f(0)=f^{\prime}(0)=0 .
$$

Moreover, we assume that $f^{\prime}(t)$ is decreasing for $t<0$ and increasing for $t>0$, near the origin $t=0$, and further that

$$
\sup _{|t|<\eta}\left|f^{\prime}(t)\right|<\lambda_{1}-\delta \quad \text { for some } \delta>0 \text { and } \eta>0 .
$$

Then the nonlinear operator equation (6.1) has at least four nontrivial solutions $u_{1}$, $u_{2}, u_{3}$ and $u_{4}$ for each $\lambda \in\left(\lambda_{1}, \lambda_{1}+\varepsilon\right)$ provided that $\varepsilon>0$ is sufficiently small (see Figure 1.7).

Proof The proof of Theorem 6.1 is based on an intermediate value argument just as in Landesman-Lazer [38]. The proof is divided into five steps.

Step 1: By Theorem 3.3 (Crandall-Rabinowitz), there exists a constant $\varepsilon_{1}>0$ such that the nonlinear operator equation (6.1) has two nontrivial solution curves $\Gamma_{1}$ and $\Gamma_{2}$ emanating from the point $\left(\lambda_{1}, 0\right)$. Just as in Step 4 of the proof of Theorem 1.2, we find that each small nontrivial solution of the nonlinear operator equation (6.1) is of constant sign for $\lambda \in\left[\lambda_{1}, \lambda_{2}\right)$. Moreover, under the present hypotheses, Lemmas 4.1 and 4.5 remain valid for $u$ of small norm. Hence there exists a constant $\varepsilon_{1}>0$ such that

$$
\begin{aligned}
& \Gamma_{1}=\left\{\left(\lambda, u_{1}(\lambda)\right) \in \mathbf{R} \times C(\bar{D}): \lambda_{1} \leq \lambda<\lambda_{1}+\varepsilon_{1}\right\}, \\
& \Gamma_{2}=\left\{\left(\lambda, u_{2}(\lambda)\right) \in \mathbf{R} \times C(\bar{D}): \lambda_{1} \leq \lambda<\lambda_{1}+\varepsilon_{1}\right\} .
\end{aligned}
$$

Here $\Gamma_{1}$ is the negative solution curve of the nonlinear operator equation (6.1) and $\Gamma_{2}$ is the positive solution curve of the nonlinear operator equation (6.1), respectively (see Figures 1.5 and 1.6). 
It should be emphasized that $u_{1}(\lambda)$ and $u_{2}(\lambda)$ are regular points of the map $G(\lambda, \cdot)$ for every $\lambda \in\left(\lambda_{1}, \lambda_{1}+\varepsilon_{1}\right)$ (see Lemma 4.1).

Step 2: Now we apply the Lyapunov-Schmidt procedure to the nonlinear operator equation (6.1), just as in Section 5 (see Theorem 5.1). Let $e_{1}(x)$ be the normalized positive solution of the equation

$$
e_{1}-\lambda_{1} K e_{1}=0 \quad \text { in } C(\bar{D})
$$

that is,

$$
\begin{cases}A e_{1}=\lambda_{1} e_{1} & \text { in } D \\ B e_{1}=0 & \text { on } \partial D \\ \left\|e_{1}\right\|_{L^{2}(D)}=1 . & \end{cases}
$$

Since the eigenvalue $\lambda_{1}$ is algebraically simple, we have the orthogonal decomposition

$$
C(\bar{D})=N\left(I-\lambda_{1} K\right) \oplus R\left(I-\lambda_{1} K\right)=\operatorname{span}\left[e_{1}\right] \oplus W,
$$

where (see Figure 5.1)

$$
\begin{aligned}
& \operatorname{span}\left[e_{1}\right]=N\left(I-\lambda_{1} K\right), \\
& W=R\left(I-\lambda_{1} K\right) .
\end{aligned}
$$

By Mazur's theorem ([74, Chapter IV, Section 6, Corollary $]$ ), there exists a continuous linear functional $e_{1}^{*}$ such that

$$
\begin{aligned}
& \left\langle e_{1}, e_{1}^{*}\right\rangle=1, \\
& \left\langle w, e_{1}^{*}\right\rangle=0 \quad \text { for all } w \in W .
\end{aligned}
$$

More concretely, we have the formula

$$
\left\langle u, e_{1}^{*}\right\rangle=\int_{D} u(x) e_{1}(x) d x \text { for all } u \in C(\bar{D}),
$$

since $e_{1}(x)$ is normalized.

If we define the orthogonal projection $Q$ from $C(\bar{D})$ onto $W$ along $N\left(I-\lambda_{1} K\right)$ by the formula (see Figure 5.1)

$$
Q u=u-\left(\int_{D} u(x) e_{1}(x) d x\right) e_{1} \quad \text { for all } u \in C(\bar{D}),
$$

or equivalently,

$$
(I-Q) u=\left(\int_{D} u(x) e_{1}(x) d x\right) e_{1}=\left\langle u, e_{1}^{*}\right\rangle e_{1} \quad \text { for all } u \in C(\bar{D}),
$$

then it is easy to see that

$$
W=R\left(I-\lambda_{1} K\right)=\left\{w \in C(\bar{D}): \int_{D} w(x) e_{1}(x) d x=0\right\} .
$$

Moreover, it follows from the orthogonal decomposition (5.5) and formula (6.3) that every function $u \in C(\bar{D})$ can be written uniquely in the form (see Figure 5.1)

$$
u=t e_{1}+w(t) \quad \text { for } t=\left\langle u, e_{1}^{*}\right\rangle \text { and } w(t) \in W .
$$


Hence we have, by formulas (6.5) and (6.4),

$$
\begin{aligned}
& \begin{cases}A u-\lambda u+f(u)=0 & \text { in } D, \\
B u=0 & \text { in } \partial D\end{cases} \\
& \Longleftrightarrow \\
& u-\lambda K u+K(f(u))=0 \quad \text { in } C(\bar{D}) \\
& \Longleftrightarrow \\
& \left\{\begin{array}{l}
u=t e_{1}+w(t), \\
w-\lambda K w(t)+Q\left(K\left(f\left(t e_{1}+w(t)\right)\right)\right)=0 \quad \text { in } C(\bar{D}), \\
t\left(1-\frac{\lambda}{\lambda_{1}}\right)+\int_{D} K\left(f\left(t e_{1}+w(t)\right)\right) \cdot e_{1}(x) d x=0
\end{array}\right.
\end{aligned}
$$

since $K(W) \subset W$.

Summing up, we are reduced to the study of the following system:

$$
\begin{aligned}
& w(t)-\lambda K w(t)+Q\left(K\left(f\left(t e_{1}+w(t)\right)\right)\right)=0 \quad \text { in } C(\bar{D}), \\
& \Psi(t, w(t)):=\left(1-\frac{\lambda}{\lambda_{1}}\right) t+\left\langle K\left(f\left(t e_{1}+w(t)\right)\right), e_{1}^{*}\right\rangle=0 .
\end{aligned}
$$

Step 3: Since $I-\lambda_{1} K$ is an isomorphism of $W$ onto itself, it follows from an application of the implicit function theorem (Theorem 3.1) that there exists a constant $\varepsilon_{2}>0$ such that if $|t|<\varepsilon_{2}$ and $\left|\lambda-\lambda_{1}\right|<\varepsilon_{2}$, then the infinite-dimensional equation (6.7a) has a unique solution $w=w(t, \lambda) \in C(\bar{D})$ :

$$
\begin{aligned}
& w(t, \lambda)-\lambda K w(t, \lambda)+Q\left(K\left(f\left(t e_{1}+w(t, \lambda)\right)\right)\right)=0 \quad \text { in } C(\bar{D}), \\
& \quad \text { for }|t|<\varepsilon_{2} \text { and }\left|\lambda-\lambda_{1}\right|<\varepsilon_{2} .
\end{aligned}
$$

If we take $\lambda \in\left(\lambda_{1}, \lambda_{1}+\varepsilon_{1}\right)$, then we find from the one-dimensional equation (6.7b) and the infinite-dimensional equation (6.8) that all small solutions

$$
u=t e_{1}+w(t, \lambda)
$$

of the nonlinear operator equation (6.1) are in one-to-one correspondence with the solutions $t$ of the one-dimensional equation

$$
\begin{aligned}
\Phi(t) & :=\Psi(t, w(t, \lambda))=t\left(1-\frac{\lambda}{\lambda_{1}}\right)+\left\langle K\left(f\left(t e_{1}+w(t, \lambda)\right)\right), e_{1}^{*}\right\rangle \\
& =t\left(1-\frac{\lambda}{\lambda_{1}}\right)+\int_{D} K\left(f\left(t e_{1}+w(t, \lambda)\right)\right) \cdot e_{1}(x) d x=0 \text { for }|t|<\varepsilon_{2} .
\end{aligned}
$$

Rephrased, the set of nontrivial solutions of the nonlinear operator equation (6.1) consists of two $C^{1}$ curves $\Gamma_{1}$ and $\Gamma_{2}$ that can be parametrized by $t$, for $t<0$ and for $t>0$, respectively.

Therefore, we obtain that the one-dimensional equation (6.9)

$$
\Phi(t)=\Psi(t, w(t, \lambda))=0
$$

has at least two nontrivial solutions, $t_{1}=t_{1}(\lambda)<0$ and $t_{2}=t_{2}(\lambda)>0$ :

$$
\Phi\left(t_{1}\right)=0, \quad \Phi\left(t_{2}\right)=0,
$$


provided that $\lambda \in\left(\lambda_{1}, \lambda_{1}+\varepsilon\right)$. Here

$$
\varepsilon=\min \left\{\varepsilon_{1}, \varepsilon_{2}\right\}
$$

In this way, we find from formulas (6.6), (6.8) and (6.9) that the nonlinear operator equation (6.1) has at least two nontrivial solutions $u_{1}$ and $u_{2}$ for each $\lambda \in\left(\lambda_{1}, \lambda_{1}+\varepsilon\right)$ :

$$
\begin{cases}u_{1}=t_{1}(\lambda) e_{1}+w\left(t_{1}(\lambda), \lambda\right), & t_{1}(\lambda)<0 \\ u_{2}=t_{2}(\lambda) e_{1}+w\left(t_{2}(\lambda), \lambda\right), & t_{2}(\lambda)>0\end{cases}
$$

Step 4: Now we show that the nonlinear operator equation (6.1) possesses a third nontrivial solution

$$
u_{3}=t_{3} e_{1}+w_{3}, \quad t_{3}>0, t_{3} \neq t_{2}, \quad w_{3}=w_{3}(\lambda) \in W .
$$

Without loss of generality, we may assume that

$$
\Phi(t) \neq 0 \quad \text { for all } t \in\left(0, \varepsilon_{2}\right) \text { and } t \neq t_{2} .
$$

In fact, if $\Phi\left(t_{3}\right)=0$ for some $t_{3} \in\left(0, \varepsilon_{2}\right)$ and $t \neq t_{2}$, then we obtain the third solution by setting

$$
u_{3}=t_{3} e_{1}+w\left(t_{3}\right) .
$$

First, we prove the following claim:

Claim 6.1 If $\lambda>\lambda_{1}$, then the function $\Phi(t)$ satisfies the conditions

$$
\left\{\begin{array}{l}
\Phi(0)=0, \Phi^{\prime}(0)<0 \\
\Phi\left(t_{2}\right)=0, \Phi^{\prime}\left(t_{2}\right) \neq 0 .
\end{array}\right.
$$

Proof Since $f(0)=0$, we have, by formula (6.9),

$$
\left\{\begin{array}{l}
\Phi(0)=\int_{D} K(f(0)) \cdot e_{1}(x) d x=0 \\
\Phi\left(t_{2}\right)=t_{2}\left(1-\frac{\lambda}{\lambda_{1}}\right)+\int_{D} K\left(f\left(u_{2}\left(t_{2}\right)\right)\right) \cdot e_{1}(x) d x=0 .
\end{array}\right.
$$

Moreover, we have the formula for the derivative $\Phi^{\prime}(t)$ of $\Phi(t)$

$$
\Phi^{\prime}(t)=1-\frac{\lambda}{\lambda_{1}}+\int_{D} K\left(f^{\prime}\left(t e_{1}+w(t, \lambda)\right)\right)\left(e_{1}+w_{t}(t, \lambda)\right) \cdot e_{1}(x) d x .
$$

Since $f^{\prime}(0)=0$, this proves that

$$
\begin{aligned}
\Phi^{\prime}(0) & =1-\frac{\lambda}{\lambda_{1}}+\int_{D} K\left(f^{\prime}(0)\right)\left(e_{1}+w_{t}(0, \lambda)\right) \cdot e_{1}(x) d x=1-\frac{\lambda}{\lambda_{1}} \\
& <0 \quad \text { for } \lambda>\lambda_{1} .
\end{aligned}
$$

Recall that the solution

$$
u_{2}(t)=u_{2}(t, \lambda)=t e_{1}+w(t, \lambda)
$$


is a regular point of the map $G(\lambda, \cdot)$ for each $\lambda \in\left(\lambda_{1}, \lambda_{1}+\varepsilon_{1}\right)$, so that the partial Fréchet derivative

$$
G_{u}\left(\lambda, u_{2}(t)\right)=I-\lambda K+K\left(f^{\prime}\left(u_{2}(t)\right)\right): C(\bar{D}) \longrightarrow C(\bar{D})
$$

is an isomorphism for each $\lambda \in\left(\lambda_{1}, \lambda_{1}+\varepsilon_{1}\right)$.

On the other hand, by differentiating the equation

$$
G\left(\lambda, u_{2}(t)\right)=u_{2}(t)-\lambda K u_{2}(t)+K\left(f\left(u_{2}(t)\right)\right)
$$

with respect to $t$, we obtain that

$$
u_{2}^{\prime}(t)-\lambda K u_{2}^{\prime}(t)+K\left(f^{\prime}\left(u_{2}(t)\right)\right) \cdot u_{2}^{\prime}(t)=G_{u}\left(\lambda, u_{2}(t)\right) \cdot u_{2}^{\prime}(t) \neq 0,
$$

since we have the assertion

$$
u_{2}^{\prime}(t)=e_{1}+w_{t}(t, \lambda) \neq 0 \quad \text { for } w_{t}(t, \lambda) \in W .
$$

Therefore, we find from assertions (6.10) and (6.11) that

$$
\begin{aligned}
\Phi^{\prime}\left(t_{2}\right) & =1-\frac{\lambda}{\lambda_{1}}+\int_{D} K\left(f^{\prime}\left(t_{2} e_{1}+w\left(t_{2}, \lambda\right)\right)\right)\left(e_{1}+w_{t}\left(t_{2}, \lambda\right)\right) \cdot e_{1}(x) d x \\
& =1-\frac{\lambda}{\lambda_{1}}+\int_{D} K\left(f^{\prime}\left(u_{2}\left(t_{2}\right)\right)\right) \cdot u^{\prime}\left(t_{2}\right) \cdot e_{1}(x) d x \\
& \neq 1-\frac{\lambda}{\lambda_{1}}+\int_{D}\left\{\lambda K\left(e_{1}+w_{t}\left(t_{2}, \lambda\right)\right)-e_{1}-w_{t}\left(t_{2}, \lambda\right)\right\} \cdot e_{1}(x) d x \\
& =1-\frac{\lambda}{\lambda_{1}}+\frac{\lambda}{\lambda_{1}} \int_{D} e_{1}(x)^{2}-\int_{D} e_{1}(x)^{2} d x=1-\frac{\lambda}{\lambda_{1}}+\frac{\lambda}{\lambda_{1}}-1 \\
& =0 \text { for } \lambda>\lambda_{1} .
\end{aligned}
$$

Indeed, it suffices to note that

$$
W=\left\{w \in C(\bar{D}): \int_{D} w(x) e_{1}(x) d x=0\right\}
$$

and further that $K: W \rightarrow W$.

The proof of Claim 6.1 is complete.

By Claim 6.1, it follows that

$$
\Phi\left(\widetilde{t}_{2}\right)=\Psi\left(\widetilde{t_{2}}, w\left(\widetilde{t_{2}}\right)\right)>0 \quad \text { for some number } \widetilde{t}_{2} \in\left(t_{2}, \varepsilon_{2}\right) .
$$

We let

$$
\Sigma:=\left\{(t, w) \in \mathbf{R} \times W: w-\lambda K w+Q\left(K\left(f\left(t e_{1}+w\right)\right)\right)=0\right\} .
$$

It should be noticed that if $t \in\left(-\varepsilon_{2}, \varepsilon_{2}\right)$, then $(t, w) \in \Sigma$ if and only if $w=w(t)$. In particular, we have the assertion

$$
\left(\widetilde{t}_{2}, w\left(\widetilde{t}_{2}\right)\right) \in \Sigma .
$$

Since $f$ is bounded on $\mathbf{R}$, there is a constant $C>0$ such that

$$
\|K(f(u))\| \leq C \quad \text { for all } u \in C(\bar{D}) .
$$


Moreover, by applying Schwarz's inequality we obtain that

$$
\begin{aligned}
\left|\int_{D} K(f(u(x))) \cdot e_{1}(x) d x\right| & \leq C \int_{D} e_{1}(x) d x \leq C \sqrt{\int_{D} d x} \cdot \sqrt{\int_{D} e_{1}(x)^{2} d x} \\
& =C \sqrt{|D|} \text { for all } u \in C(\bar{D}),
\end{aligned}
$$

where $|D|$ is the volume of the bounded domain $D$.

Hence we have, for all $w \in W$,

$$
\begin{aligned}
\Psi\left(\widetilde{t}_{3}, w\right) & =\widetilde{t}_{3}\left(1-\frac{\lambda}{\lambda_{1}}\right)+\int_{D} K\left(f\left(\widetilde{t}_{3} e_{1}+w(x)\right)\right) \cdot e_{1}(x) d x \\
& <\widetilde{t}_{3}\left(1-\frac{\lambda}{\lambda_{1}}\right)+C \sqrt{|D|}<0
\end{aligned}
$$

if we take a large number $\widetilde{t}_{3}>\widetilde{t}_{2}$ such that

$$
\tilde{t}_{3}>\left(\frac{\lambda_{1}}{\lambda-\lambda_{1}}\right) C \sqrt{|D|}
$$

Furthermore, by arguing just as in Amann-Ambrosetti-Mancini [7, Lemma 1.2] we can prove the following lemma:

Lemma 6.1 For every $\alpha>0$, there exists a connected subset $\Sigma(\alpha)$ of $\Sigma$ such that

$$
\operatorname{proj}_{\mathbf{R}} \Sigma(\alpha) \supset[-\alpha, \alpha] .
$$

Proof First, we remark that the mapping

$$
\begin{aligned}
g(t, w): \mathbf{R} \times W & \longrightarrow W \\
(t, w) & \longmapsto(I-\lambda K)^{-1} Q\left(K\left(f\left(t e_{1}+w\right)\right)\right)
\end{aligned}
$$

is uniformly bounded and compact on bounded sets, for each $\lambda \in\left(\lambda_{1}, \lambda_{1}+\varepsilon_{1}\right)$. Moreover, the image $f(X)$ is bounded in the space $X=C(\bar{D})$, since the function $f$ is bounded on $\mathbf{R}$.

Now we choose a constant $r>0$ so large that

$$
\|g(t, w)\|<r \quad \text { for all }(t, w) \in \mathbf{R} \times W .
$$

If we denote by $B(0, r)$ the open ball about 0 in $W$ with radius $r$, then it is easy to see that

$$
\begin{aligned}
\Sigma & =\left\{(t, w) \in \mathbf{R} \times W: w-\lambda K w+Q\left(K\left(f\left(t e_{1}+w\right)\right)\right)=0\right\} \\
& =\{(t, w) \in \mathbf{R} \times W: w+g(t, w)=0\} \\
& \subset \mathbf{R} \times B(0, r) .
\end{aligned}
$$

Hence we can define the Leray-Schauder degree deg $(G(t, \cdot), B(0, r), 0)$ for a map $G=I+g$ defined by the formula

$$
\begin{aligned}
G: \mathbf{R} \times W & \longrightarrow W \\
(t, w) & \longmapsto w+g(t, w) .
\end{aligned}
$$


Then, by a homotopic argument it follows that

$$
\operatorname{deg}(G(t, \cdot), B(0, r), 0)=\operatorname{deg}\left(I_{W}, B(0, r), 0\right)=1 \quad \text { for every } t \in \mathbf{R},
$$

where $I_{W}$ is the identity map on the space $W$.

Hence, we have the assertion

$$
\operatorname{proj}_{\mathbf{R}} \Sigma=\mathbf{R} \text {. }
$$

In order to show that, for every $\alpha>0$ there is a connected subset $\Sigma(\alpha)$ with the stated properties, we follow a well-known argument used in bifurcation theory (see [22, Chapter 19]). For $\alpha>0$, we let

$$
A:=[-\alpha, \alpha] \times \overline{B(0, r)} .
$$

Furthermore, we let

$$
\begin{aligned}
& K:=\Sigma \cap A, \\
& K_{ \pm}:=\Sigma \cap(\{ \pm \alpha\} \times \overline{B(0, r)}) .
\end{aligned}
$$

Then $K$ is a compact metric space and $K_{ \pm}$are non-empty disjoint, closed subsets of $K$.

Our proof is based on a reduction to absurdity. Assume, to the contrary, that there does not exist a connected subset of $K$ joining $K_{+}$and $K_{-}$. Then it follows from an application of the separation theorem ([22, Theorem 19.4]) that there exist disjoint compact sets $C_{ \pm} \supset K_{ \pm}$such that

$$
K=C_{+} \cup C_{-} .
$$

We remark that

$$
C_{-} \cap\left(C_{+} \cup([-\alpha, \alpha] \times S(0, r)) \cup(\{\alpha\} \times \overline{B(0, r)})\right)=\emptyset,
$$

where $S(0, r)$ is the surface of the ball $B(0, r)$. Hence we can find an open subset $U$ of $A$ such that

- $C_{-} \subset U$,

- $\bar{U} \cap\left(C_{+} \cup([-\alpha, \alpha] \times S(0, r)) \cup(\{\alpha\} \times \overline{B(0, r)})\right)=\emptyset$.

This implies that

$$
G(t, w) \neq 0 \quad \text { for all }(t, w) \text { in the boundary of } U \text { relative to } A,
$$

and that

$$
U_{\alpha}:=\{w \in W:(\alpha, w) \in U\}=\emptyset .
$$

Therefore, by the excision property and the homotopy invariance property of the Leray-Schauder degree we obtain that

$$
\begin{aligned}
1 & =\operatorname{deg}(G(-\alpha, \cdot), B(0, r), 0)=\operatorname{deg}\left(G(-\alpha, \cdot), U_{-\alpha}, 0\right)=\operatorname{deg}\left(G(\alpha, \cdot), U_{\alpha}, 0\right) \\
& =0 .
\end{aligned}
$$


This contradiction proves the existence of a connected subset $\Sigma(\alpha)$ of $\Sigma$ joining $K_{+}$and $K_{-}$.

Summing up, we have proved the desired assertion

$$
\operatorname{proj}_{\mathbf{R}} \Sigma \supset[-\alpha, \alpha]
$$

The proof of Lemma 6.1 is complete.

By applying Lemma 6.1 with $\alpha:=\widetilde{t}_{3}$, we can find a connected subset $\Sigma\left(\widetilde{t}_{3}\right)$ of $\Sigma$ such that

$$
\operatorname{proj}_{\mathbf{R}} \Sigma\left(\widetilde{t}_{3}\right) \supset\left[-\widetilde{t}_{3}, \widetilde{t}_{3}\right] \supset\left[-\widetilde{t}_{2}, \widetilde{t}_{2}\right]
$$

Hence we have the assertions

$$
\left\{\begin{array}{l}
\left(\widetilde{t}_{2}, w\left(\widetilde{t}_{2}\right)\right) \in \Sigma_{1}, \\
\left(\widetilde{t}_{3}, \widetilde{w}_{3}\right) \in \Sigma_{1} \quad \text { for some } \widetilde{w}_{3} \in W .
\end{array}\right.
$$

since $\left(\widetilde{t}_{2}, \widetilde{w}_{2}\right) \in \Sigma$ if and only if $\widetilde{w}_{2}=w\left(\widetilde{t}_{2}\right)$ for $\widetilde{t}_{2}<\varepsilon_{2}$.

Moreover, the mapping

$$
\Psi: \Sigma_{1} \longrightarrow \Psi(t, w)
$$

is continuous and satisfies the inequalities:

$$
\left\{\begin{array}{l}
\Psi\left(\widetilde{t}_{2}, w\left(\widetilde{t}_{2}\right)\right)=\Phi\left(\widetilde{t}_{2}\right)>0, \\
\Psi\left(\widetilde{t}_{3}, \widetilde{w}_{3}\right)<0 .
\end{array}\right.
$$

Therefore, by an intermediate value argument we find a point $\left(t_{3}, w_{3}\right) \in \Sigma_{1}$ such that

$$
\left\{\begin{array}{l}
\tilde{t}_{2}<t_{3} \leq \tilde{t}_{3} \\
\Psi\left(t_{3}, w_{3}\right)=0 .
\end{array}\right.
$$

In this way, we have found the third solution by setting

$$
u_{3}=t_{3} e_{1}+w_{3} \quad \text { for } t_{3}>t_{2} \text { and } w_{3} \in W \text {. }
$$

Step 5: The fourth solution

$$
u_{4}=t_{4} e_{1}+w_{4} \quad \text { for } t_{4}<t_{1} \text { and } w_{4} \in W
$$

can be obtained in an analogous way.

Now the proof of Theorem 6.1 is complete. 


\section{Proof of Theorem 1.4}

In this last section we consider the case where $f(t)$ and $f^{\prime}(t)$ are bounded on $\mathbf{R}$. The proof of Theorem 1.4 is carried out by using the global theory of positive mappings due to Dancer [27] (Theorem 3.4).

More precisely, we prove the following generalization of Szulkin [52, Theorem $5.2]$ to the degenerate case:

Theorem 7.1 Assume that hypotheses (H.1) and (H.2) are satisfied and let $f(t)$ be a $C^{1}$ function on $\mathbf{R}$ such that $f(0)=0$ and $f^{\prime}(0)=0$. Then we have the following two assertions:

(i) If, in addition, there exists a constant $k>0$ such that

$$
0 \leq f^{\prime}(t) \leq k<\min \left\{\lambda_{1}, \lambda_{2}-\lambda_{1}\right\}
$$

then the set of nontrivial solutions of the nonlinear operator equation (4.1), bifurcating at $\left(\lambda_{1}, 0\right)$, consists of two $C^{1}$ branches, one positive $\Gamma_{1}$ and one negative $\Gamma_{2}$. These branches are given respectively by the formulas (see Figure 1.8)

$$
\begin{aligned}
& \Gamma_{1}=\left\{(\lambda, u) \in \mathbf{R} \times C(\bar{D}): \lambda=\lambda^{(1)}(s), u=u_{1}(s) \quad \text { for } 0 \leq s<\infty\right\}, \\
& \left(\lambda^{(1)}(0), u_{1}(0)\right)=\left(\lambda_{1}, 0\right),
\end{aligned}
$$

and

$$
\begin{aligned}
& \Gamma_{2}=\left\{(\lambda, u) \in \mathbf{R} \times C(\bar{D}): \lambda=\lambda^{(2)}(s), u=u_{2}(s) \quad \text { for } 0 \leq s<\infty\right\}, \\
& \left(\lambda^{(2)}(0), u_{2}(0)\right)=\left(\lambda_{1}, 0\right) .
\end{aligned}
$$

(ii) Furthermore, if $f(t)$ is bounded, it follows that

$$
\begin{aligned}
& \lambda^{(1)}(s) \longrightarrow \lambda_{1} \quad \text { as } s \rightarrow \infty, \\
& \lambda^{(2)}(s) \longrightarrow \lambda_{1} \quad \text { as } s \rightarrow \infty .
\end{aligned}
$$

Remark 7.1 The behavior of $\Gamma_{1}$ and $\Gamma_{2}$ for a bounded function $f(t)$ is in sharp contrast with that described in Theorem 1.2. More precisely, the branches turn back towards $\lambda_{1}$, hence they cannot be parametrized by $\lambda$.

Proof By Theorem 3.3 (Crandall-Rabinowitz), it follows that there exist precisely two branches of solutions emanating from the point $\left(\lambda_{1}, 0\right)$. We shall show the conclusions of the theorem hold true for the positive branch $\Gamma_{1}$, since the proof for the negative one $\Gamma_{2}$ is analogous.

The proof of Theorem 7.1 is divided into four steps.

Step 1: Take a real-valued, smooth function $g(\lambda)$ on $\mathbf{R}$ such that $0 \leq g(\lambda) \leq 1$ on $\mathbf{R}$ and that

$$
g(\lambda)= \begin{cases}0 & \text { for } \lambda \leq \lambda_{1}-\delta \\ 1 & \text { for } \lambda \geq \lambda_{1}\end{cases}
$$

We define a mapping

$$
G_{1}(\lambda, u): \mathbf{R} \times C(\bar{D}) \longrightarrow C(\bar{D})
$$


by the formula

$$
G_{1}(\lambda, u)=u-\lambda K u+g(\lambda) K(f(u)) \quad \text { for }(\lambda, u) \in \mathbf{R} \times C(\bar{D}) .
$$

We remark that

$$
\frac{g(\lambda) K(f(u))}{\|u\|} \longrightarrow 0 \quad \text { as } u \rightarrow 0 \text { in } P \backslash\{0\} \text {, locally uniformly in } \lambda \in \mathbf{R} .
$$

Hence, by applying Theorem 3.4 due to Dancer [27] to our situation we find that the set of positive solutions of the operator equation $G_{1}(\lambda, u)=0$ contains an unbounded subcontinuum $\Gamma_{1}$ emanating from $\left(\lambda_{1}, 0\right)$.

Step 2: We consider the case where $0<\lambda<\lambda_{1}$ : If condition (1.5) is satisfied, then we have, for $0<\lambda<\lambda_{1}$,

$$
\lambda-g(\lambda) \frac{f(u)}{u} \geq \begin{cases}\lambda & \text { for } 0<\lambda \leq k, \\ \lambda-k & \text { for } k<\lambda<\lambda_{1} .\end{cases}
$$

Hence it follows from an application of Proposition 4.2 that

$$
\mu_{1}\left(\lambda-g(\lambda) \frac{f(u)}{u}\right) \leq \mu_{1}(\lambda)=\frac{\lambda}{\lambda_{1}}<1 \quad \text { for } 0<\lambda<\lambda_{1}
$$

This implies that the eigenvalue problem with weight $\lambda-g(\lambda) f(u) / u$

$$
\left\{\begin{array}{l}
K\left(\lambda-g(\lambda) \frac{f(u)}{u}\right) v=\mu v \\
v \in P
\end{array}\right.
$$

does not have an eigenvalue 1. In other words, the operator equation

$$
G_{1}(\lambda, u)=u-K\left(\lambda-g(\lambda) \frac{f(u)}{u}\right) u=0
$$

has only the trivial solution for $0<\lambda<\lambda_{1}$.

Therefore, we have the assertion

$$
\Gamma_{1} \cap\left\{(\lambda, u): 0<\lambda<\lambda_{1}\right\}=\emptyset
$$

Step 3: We consider the case where $\lambda>\lambda_{1}$ : Remark that we have the formula

$$
\begin{aligned}
G(\lambda, u) & =u-\lambda K u+K(f(u))=u-\lambda K u+g(\lambda) K(f(u)) \\
& =G_{1}(\lambda, u)=0 \quad \text { for }(\lambda, u) \in \Gamma_{1} .
\end{aligned}
$$

Substep (3-1): The case where $\lambda=\lambda_{2}$. Since we have, by condition (1.5),

$$
\lambda_{1}<\lambda_{2}-\frac{f(t)}{t} \quad \text { for } t \in \mathbf{R}
$$

it follows that

$$
\mu_{1}\left(\lambda_{2}-\frac{f(u)}{u}\right)>\mu_{1}\left(\lambda_{1}\right)=1
$$


This implies that the eigenvalue problem with weight $\lambda_{2}-f(u) / u$

$$
\left\{\begin{array}{l}
K\left(\lambda_{2}-\frac{f(u)}{u}\right) v=\mu v, \\
v \in P
\end{array}\right.
$$

does not have an eigenvalue 1 . In other words, the equation

$$
G(\lambda, u)=u-K\left(\lambda-\frac{f(u)}{u}\right) u=0
$$

has no positive solutions for $\lambda=\lambda_{2}$.

Therefore, we have proved that the subcontinuum $\Gamma_{1}$ is bounded away from the hyperplane $\left\{\lambda=\lambda_{2}\right\}$ (see Figure 1.4), since we have, by condition (1.5),

$$
\frac{f(t)}{t} \leq k<\lambda_{2}-\lambda_{1} \quad \text { for all } t \in \mathbf{R} .
$$

Substep (3-2): The case where $\lambda_{1}<\lambda<\lambda_{2}$. Then we have, for $\lambda_{1}<\lambda<\lambda_{2}$,

$$
\lambda-f^{\prime}(u) \geq \lambda-k>0 .
$$

Hence it follows from an application of Proposition 4.2 that

$$
\mu_{2}\left(\lambda-f^{\prime}(u)\right) \leq \mu_{2}(\lambda)=\frac{\lambda}{\lambda_{2}}<1 \quad \text { for } \lambda_{1}<\lambda<\lambda_{2} .
$$

We are reduced to the study of the following three cases:

(a) $\mu_{2}\left(\lambda-f^{\prime}(u)\right)<\mu_{1}\left(\lambda-f^{\prime}(u)\right)<1$ for $(\lambda, u) \in \Gamma_{1}$ with $\lambda<\lambda_{2}$.

(b) $\mu_{2}\left(\lambda-f^{\prime}(u)\right)<1<\mu_{1}\left(\lambda-f^{\prime}(u)\right)$ for $(\lambda, u) \in \Gamma_{1}$ with $\lambda<\lambda_{2}$.

(c) $\mu_{2}\left(\lambda-f^{\prime}(u)\right)<1=\mu_{1}\left(\lambda-f^{\prime}(u)\right)$ for $(\lambda, u) \in \Gamma_{1}$ with $\lambda<\lambda_{2}$.

In order to prove part (i) of Theorem 7.1, it suffices to show that

The set $\Gamma_{1} \backslash\left\{\left(\lambda_{1}, 0\right)\right\}$ is locally homeomorphic to the set of real numbers. (7.5)

We shall show that the Fréchet derivative

$$
G^{\prime}(\lambda, u)=\left(-K u, I-\left(K\left(\lambda-f^{\prime}(u)\right)\right): \mathbf{R} \times C(\bar{D}) \longrightarrow C(\bar{D})\right.
$$

is an epimorphism (a surjective function). Here

$$
G^{\prime}(\lambda, u)(\mu, v)=-\mu K u+v-K\left(\lambda-f^{\prime}(u)\right) v \quad \text { for }(\mu, v) \in \mathbf{R} \times C(\bar{D}) .
$$

The situation can be visualized as in Figure 7.1 and assertion (7.6) below.

Cases (a) and (b): Since $\mu_{1}\left(\lambda-f^{\prime}(u)\right) \neq 1$ for $(\lambda, u) \in \Gamma_{1}$, it follows that the partial Fréchet derivative

$$
G_{u}(\cdot, u)=I-K\left(\lambda-f^{\prime}(u)\right)
$$

is an isomorphism. In other words, $u$ is a regular point of the mapping $G(\lambda, u)$.

Case (c): If $\mu_{1}\left(\lambda-f^{\prime}(u)\right)=1$ for $(\lambda, u) \in \Gamma_{1}$, it follows from an application of Proposition 4.1 that the eigenvalue $\mu_{1}\left(\lambda-f^{\prime}(u)\right)$ is algebraically simple. However, since the operator $K\left(\lambda-f^{\prime}(u)\right)$ is compact, we have the formula

$$
\text { ind } G_{u}(\lambda, u)=\text { ind }\left(I-K\left(\lambda-f^{\prime}(u)\right)\right)=0 .
$$

This implies that

$$
\operatorname{codim} R\left(G_{u}(\lambda, u)\right)=\operatorname{dim} N\left(G_{u}(\lambda, u)\right)=1 .
$$

More precisely, we have the following claim: 


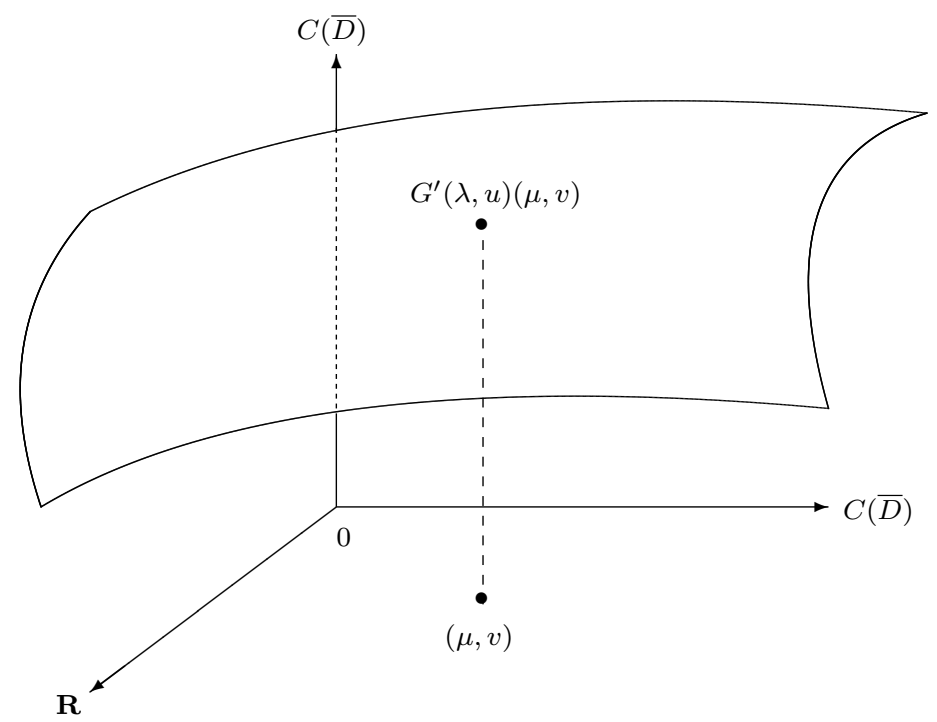

Fig. 7.1 The derivative $G^{\prime}(\lambda, u)=\left(-K u, I-K\left(\lambda-f^{\prime}(u)\right)\right)$ is an epimorphism

Claim 7.1 The function $K u \in \operatorname{Int}\left(P_{e}\right)$ and

$$
K u \notin R\left(G_{u}(\lambda, u)\right) .
$$

In other words, we have the assertion (see Figure 7.2)

$$
C(\bar{D})=R\left(G_{u}(\lambda, u)\right) \oplus N\left(G_{u}(\lambda, u)\right)=R\left(G_{u}(\lambda, u)\right) \dot{+} \operatorname{span}[K u] .
$$

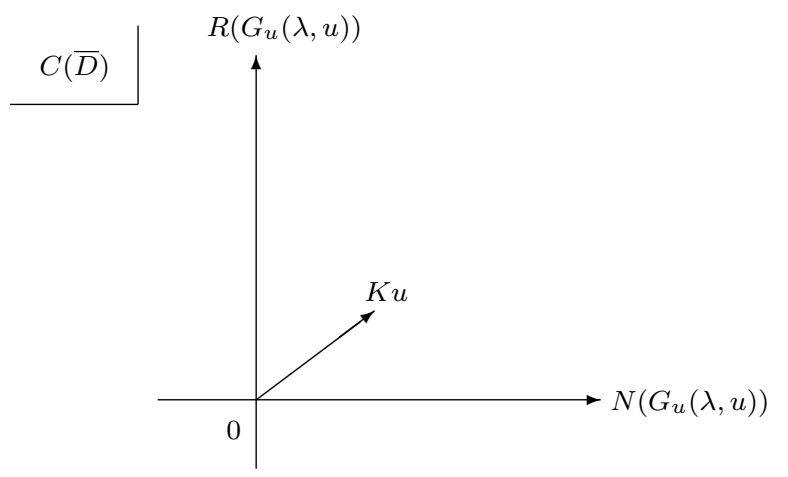

Fig. 7.2 The function $K u \in \operatorname{Int}\left(P_{e}\right)$ and $K u \notin R\left(G_{u}(\lambda, u)\right)$

Proof First, by the strong positivity of $K$ it follows that

$$
K u \in \operatorname{Int}\left(P_{e}\right) .
$$


Our proof is based on a reduction to absurdity. We assume, to the contrary, that

$$
K u \in R\left(G_{u}(\lambda, u)\right)
$$

Then it follows that

$$
K u=G_{u}(\lambda, u) v=v-K\left(\left(\lambda-f^{\prime}(u)\right) v\right) \quad \text { for some } v \in C(\bar{D}) .
$$

We remark that

$$
v=K u-\lambda K v+K\left(f^{\prime}(u) v\right) \in C_{e}(\bar{D}),
$$

since the operator $K$ maps $C(\bar{D})$ into $C_{e}(\bar{D})$.

We introduce a strongly positive, compact linear operator

$$
T: C_{e}(\bar{D}) \longrightarrow C_{e}(\bar{D})
$$

by the formula

$$
T:=\left.K\left(\left(\lambda-f^{\prime}(u)\right) v\right)\right|_{C_{e}(\bar{D})} .
$$

Then the operator $T$ has an eigenvalue 1 , since $\mu_{1}\left(\lambda-f^{\prime}(u)\right)=1$.

Therefore, by using the Krel̆n-Rutman theorem (Theorem 2.5) we can find a linear functional $\psi^{*} \in C_{e}(\bar{D})^{*}$ such that

$$
\left\{\begin{array}{l}
T^{*} \psi^{*}=\psi^{*} \\
\left\langle z, \psi^{*}\right\rangle>0 \text { for all } z \in P_{e} \backslash\{0\} .
\end{array}\right.
$$

In particular, we have the assertion

$$
\left\langle v-K\left(\lambda-f^{\prime}(u)\right) v, \psi^{*}\right\rangle=\left\langle K u, \psi^{*}\right\rangle>0,
$$

since $K u \in P_{e} \backslash\{0\}$.

However, we have the assertion

$$
\begin{aligned}
0 & <\left\langle v-K\left(\lambda-f^{\prime}(u)\right) v, \psi^{*}\right\rangle=\left\langle v-T v, \psi^{*}\right\rangle \\
& =\left\langle v, \psi^{*}\right\rangle-\left\langle v, T^{*} \psi^{*}\right\rangle=\left\langle v, \psi^{*}\right\rangle-\left\langle v, \psi^{*}\right\rangle=0 .
\end{aligned}
$$

This is a contradiction.

The proof of Claim 7.1 is complete.

By Claim 7.1, we find that the Fréchet derivative $G^{\prime}(\lambda, u)$ is an epimorphism (a surjective function).

Summing up, by applying the implicit function theorem (Theorem 3.1) we obtain a local homeomorphism at each point $(\lambda, u) \in \Gamma_{1} \backslash\left\{\left(\lambda_{1}, 0\right)\right\}$ for $\lambda_{1}<\lambda<\lambda_{2}$. Indeed, it suffices to note the following:

$$
\begin{aligned}
& \quad(\lambda, u) \in \Gamma_{1} \backslash\left\{\left(\lambda_{1}, 0\right)\right\} \quad \text { for } \lambda_{1}<\lambda<\lambda_{2} \\
& \Longleftrightarrow \\
& \left.\quad \text { for } \lambda_{1}<\lambda, u\right)=u-\lambda K u+K(f(u))=u-\lambda K u+g(\lambda) K(f(u))=\lambda_{2} \text { and } u \neq 0 .
\end{aligned}
$$

Part (i) of Theorem 7.1 follows from assertions (7.4) and (7.5). Namely, we have the assertion (see Figure 1.8)

$$
(\lambda, u) \in \Gamma_{1} \backslash\left\{\left(\lambda_{1}, 0\right)\right\} \quad \text { for } \lambda_{1}<\lambda<\lambda_{2}
$$




$$
\begin{aligned}
& \Longleftrightarrow \\
& \begin{cases}\lambda=\lambda^{(1)}(s) & \text { for } 0<s<\infty, \\
u=u_{1}(s)=t_{1}(s) e_{1}+w_{1}(s) & \text { for } 0<s<\infty .\end{cases}
\end{aligned}
$$

Similarly, we have the assertion (see Figure 1.8)

$$
\begin{aligned}
& (\lambda, u) \in \Gamma_{2} \backslash\left\{\left(\lambda_{1}, 0\right)\right\} \quad \text { for } \lambda_{1}<\lambda<\lambda_{2} \\
& \Longleftrightarrow \\
& \begin{cases}\lambda=\lambda^{(2)}(s) & \text { for } 0<s<\infty, \\
u=u_{2}(s)=t_{2}(s) e_{1}+w_{2}(s) & \text { for } 0<s<\infty .\end{cases}
\end{aligned}
$$

Step 4: In order to prove part (ii) of Theorem 7.1, we apply the LyapunovSchmidt procedure to the nonlinear operator equation (4.1).

Let $e_{1}, e_{1}^{*}, W$ and $Q$ be as in Section 5. By virtue of formula (5.13), we can express uniquely the solution $u(s)$ as follows:

$$
u_{1}(s)=t_{1}(s) e_{1}+w_{1}(s), \quad w_{1}(s) \in W=\left\{\left(I-\lambda_{1} K\right) v: u \in C(\bar{D})\right\} .
$$

Here we recall the following two assertions:

(1) The positive solution branch $\Gamma_{1}$ is unbounded (see Step 1).

(2) The distance from $\Gamma_{1}$ to the hyperplane $\left\{\lambda=\lambda_{2}\right\}$ is positive (Substep (3-1)).

First, we consider the case where $w_{1}(s)$ is bounded in $C(\bar{D})$ as $s \rightarrow \infty$ and

$$
t_{1}(s) \longrightarrow \infty \text { as } s \rightarrow \infty \text {. }
$$

Then it follows from the one-dimensional equation (5.9) that

$$
\left(1-\frac{\lambda^{(1)}}{\lambda_{1}}\right) t_{1}(s)+\left\langle K\left(f\left(t_{1}(s) e_{1}+w_{1}(s)\right)\right), e_{1}^{*}\right\rangle=0 .
$$

However, we find from inequality (6.12) that if $f(t)$ is bounded, the second term on the left-hand side of this equation is bounded.

Therefore, we find that the desired assertion

$$
\lambda^{(1)}(s) \longrightarrow \lambda_{1} \quad \text { as } s \rightarrow \infty
$$

holds true.

Secondly, we consider the case where $w_{1}(s)$ is unbounded in $C(\bar{D})$ as $s \rightarrow \infty$. We have, by the operator equation (5.7),

$$
\frac{w_{1}}{\left\|w_{1}(s)\right\|}-\lambda^{(1)}(s) K\left(\frac{w_{1}(s)}{\left\|w_{1}(s)\right\|}\right)+Q\left(K\left(\frac{f\left(t e_{1}+w_{1}(s)\right)}{\left\|w_{1}(s)\right\|}\right)\right)=0 \quad \text { in } C(\bar{D}) .
$$

Since the resolvent

$$
K: C(\bar{D}) \longrightarrow C_{e}(\bar{D})
$$

is compact and strongly positive, we can find a sequence $\left\{s_{j}\right\}$, a function $\chi \in P_{e}$ and a number $\lambda_{1} \leq \gamma<\lambda_{2}$ such that, as $j \rightarrow \infty$,

$$
v\left(s_{j}\right):=\frac{w_{1}\left(s_{j}\right)}{\left\|w_{1}\left(s_{j}\right)\right\|} \in C(\bar{D}) \backslash\{0\}
$$




$$
\begin{aligned}
& K v\left(s_{j}\right) \longrightarrow \chi \quad \text { in } C_{e}(\bar{D}), \\
& \lambda^{(1)}\left(s_{j}\right) \longrightarrow \gamma .
\end{aligned}
$$

Hence, by passing to the limit in formula (7.7) we obtain from assertions (7.8) that

$$
v\left(s_{j}\right) \longrightarrow \gamma \chi \quad \text { in } C_{e}(\bar{D}) \text { as } j \rightarrow \infty,
$$

so that

$$
K v\left(s_{j}\right) \longrightarrow \gamma K \chi \quad \text { in } C_{e}(\bar{D}) \text { as } j \rightarrow \infty .
$$

Summing up, we have proved that

$$
\left\{\begin{array}{l}
\chi \in P_{e}, \quad\|\chi\|=\frac{1}{\gamma}, \\
K \chi=\frac{1}{\gamma} \chi \quad \text { in } C_{e}(\bar{D}), \\
\lambda_{1} \leq \gamma<\lambda_{2} .
\end{array}\right.
$$

By Proposition 4.1, this proves that

$$
\frac{1}{\gamma}=\mu_{1}=\frac{1}{\lambda_{1}}
$$

so that

$$
\gamma=\lambda_{1}
$$

Therefore, we find from assertion (7.8c) that the desired assertions

$$
\lambda^{(1)}\left(s_{j}\right) \longrightarrow \lambda_{1} \quad \text { as } j \rightarrow \infty
$$

hold true.

Now the proof of Theorem 7.1 is complete.

\section{The Leray-Schauder index of critical points and its application}

Let $X$ be a Banach space and let $G$ be a nonlinear map of $X$ into itself. In this last section we consider the case where $G$ is a compact perturbation of the identity map. It is known (see [23, Section 3.4]) that if $u_{0}$ is an isolated solution of the nonlinear operator equation

$$
G(u)=p \quad \text { for } p \in X,
$$

then the Leray-Schauder index $i\left(G, u_{0}\right)$ is well-defined. The purpose of this section is to show (Theorem 8.1) that if the null space $N\left(G^{\prime}\left(u_{0}\right)\right)$ is one dimensional, then we have the assertion

$$
\left|i\left(G, u_{0}\right)\right| \leq 1
$$

Finally, we apply Theorem 8.1 (or Corollary 8.1) to the semilinear elliptic boundary value problem (1.2) with $\lambda:=\lambda_{1}$ :

$$
\begin{cases}A u-\lambda_{1} u+f(u)=0 & \text { in } D, \\ B u=a\left(x^{\prime}\right) \frac{\partial u}{\partial \nu}+b\left(x^{\prime}\right) u=0 & \text { on } \partial D .\end{cases}
$$


8.1 The Leray-Schauder index calculation

Now we formulate our result more precisely. Assume that a mapping $G: X \rightarrow X$ is of class $C^{1}$ such that

$$
G=I-C \text { for some compact map } C: X \rightarrow X \text {. }
$$

Recall that $C: X \rightarrow X$ is said to be compact if it is continuous and maps bounded sets into relatively compact sets.

If $u_{0}$ is an isolated solution of the nonlinear operator equation

$$
G(u)=u-C(u)=p, \quad p \in X,
$$

then the Leray-Schauder index $i\left(G, u_{0}\right)$ is defined by the formula

$$
i\left(G, u_{0}\right)=\lim _{\varepsilon \downarrow 0} \operatorname{deg}\left(G, B\left(u_{0}, \varepsilon\right), p\right),
$$

where $B\left(u_{0}, \varepsilon\right)$ is an open ball about $u_{0}$ of radius $\varepsilon$.

It is known from the index formula (see [22, Theorem 21.10]) that

$$
i\left(G, u_{0}\right)= \pm 1
$$

provided that the Fréchet derivative $G^{\prime}\left(u_{0}\right)$ is invertible.

In this section we study the case where the null space $N\left(G^{\prime}\left(u_{0}\right)\right)$ is one dimensional. Without loss of generality, we may assume that $u_{0}=0$ and $p=0$ :

$$
i(G, 0)=\lim _{\varepsilon \downarrow 0} \operatorname{deg}(G, B(0, \varepsilon), 0) .
$$

We express the map $C$ in the form

$$
C(u)=K u-N(u) \quad \text { for } u \in X,
$$

where $K: X \rightarrow X$ is a self-adjoint, bounded linear operator and $N: X \rightarrow X$ satisfies the condition

$$
\|N(u)\|=o(\|u\|) \quad \text { as } u \rightarrow 0 \text { in } X,
$$

so that the Fréchet derivative $G^{\prime}(0)$ is equal to the following:

$$
G^{\prime}(0)=I-K \text {. }
$$

Furthermore, we assume that $K$ has the first reciprocal eigenvalue 1 of simple algebraic multiplicity:

$$
K e_{0}=e_{0},
$$

where $e_{0}$ is an eigenvector of $K$ such that

$$
\begin{aligned}
& \left\|e_{0}\right\|=1, \\
& N(I-K)=\operatorname{span}\left[e_{0}\right] .
\end{aligned}
$$

Now, as in formula (5.5) we consider the Lyapunov-Schmidt orthogonal decomposition of $X$ :

$$
X=N(I-K) \oplus R(I-K) .
$$


By Mazur's theorem ([74, Chapter IV, Section 6, Corollary $]$ ), there exists a continuous linear functional $e_{0}^{*}$ on $X$ such that

$$
\begin{aligned}
& \left\langle e_{0}^{*}, e_{0}\right\rangle=1, \\
& \left\langle e_{0}^{*}, w\right\rangle=0 \text { for all } w \in W:=R(I-K) .
\end{aligned}
$$

Then we can express every vector $u \in X$ as follows (see Figure 8.1):

$$
u=t e_{0}+w \text { for } t=\left\langle e_{0}^{*}, u\right\rangle \text { and } w \in W .
$$

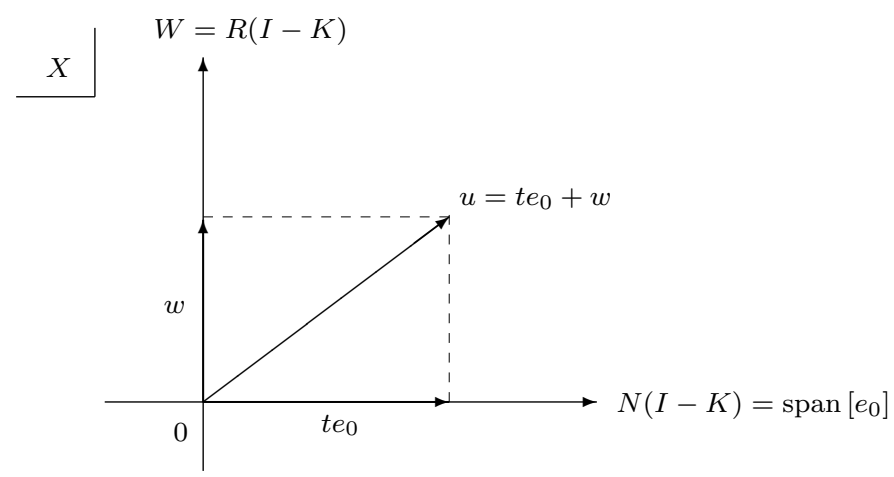

Fig. 8.1 The orthogonal decomposition (8.3) of $X$

Denote by $Q$ the projection of $X$ onto $W$ along $N(I-K)$ :

$$
Q u=u-\left\langle e_{0}^{*}, u\right\rangle e_{0} \quad \text { for } u \in X,
$$

or equivalently,

$$
(I-Q) u=\left\langle e_{0}^{*}, u\right\rangle e_{0} \quad \text { for } u \in X .
$$

Then it is easy to see that the operator equation

$$
G(u)=u-K u+N u=0 \text { for } u=t e_{0}+w,
$$

is equivalent to the following system:

$$
\begin{aligned}
& w-K w+Q N\left(t e_{0}+w\right)=0 \text { in } W, \\
& \Phi(t, w):=\left\langle N\left(t e_{0}+w\right), e_{0}^{*}\right\rangle=0 \text { in } \mathbf{R} .
\end{aligned}
$$

However, since $\|N(u)\|=o(\|u\|)$ as $u \rightarrow 0$ in $X$, it follows from an application of the implicit function theorem (Theorem 3.1) that, for each $t \in \mathbf{R}$ near the origin the equation (8.6a) has a unique solution

$$
w=\varphi(t) \in W
$$

such that the map

$$
\varphi:(-\varepsilon, \varepsilon) \longrightarrow W
$$


is of class $C^{1}$ and $\varphi(0)=\varphi^{\prime}(0)=0$. Indeed, it suffices to note that the Fréchet derivative

$$
I-K: W \longrightarrow W
$$

is an isomorphism.

Hence we have the equivalent assertions

$$
\begin{aligned}
& \left\{\begin{array}{l}
G(u)=u-K u+N(u)=0 \text { in } X, \\
u=t e_{0}+w
\end{array}\right. \\
& \Longleftrightarrow \begin{array}{ll}
w-K w+Q N\left(t e_{0}+w\right)=0 \quad \text { in } W, \\
\Phi(t, w)=\left\langle N\left(t e_{0}+w\right), e_{0}^{*}\right\rangle=0 \quad \text { in } \mathbf{R}
\end{array} \\
& \Longleftrightarrow \begin{array}{ll}
\varphi(t)-K \varphi(t)+Q N\left(t e_{0}+\varphi(t)\right)=0 & \text { in } W, \\
\Phi(t, \varphi(t))=0 & \text { in } \mathbf{R}, \\
u=t e_{0}+\varphi(t) & \text { for sufficiently small norm. }
\end{array}
\end{aligned}
$$

We remark that

$$
\Phi(t, \varphi(t)) \neq 0 \quad \text { for sufficiently small } t \text { with } t \neq 0,
$$

since $u=0$ is an isolated solution of the equation $G(u)=0$.

We are in a position to prove the following generalization of Szulkin [52, Theorem 6.1] to the degenerate case:

Theorem 8.1 Assume that $u=0$ is an isolated solution of the equation $G(u)=0$ and that

$$
X=N(I-K) \oplus R(I-K) .
$$

Then we have the assertion

$$
|i(G, 0)| \leq 1 .
$$

Furthermore, we have the product formula

$$
i(G, 0)=i\left(\left.(I-K)\right|_{W}, 0\right) \cdot i(\Phi(\cdot, \varphi(\cdot)), 0) .
$$

Proof The proof of Theorem 8.1 is divided into two steps.

Step 1: Our proof is based on the Leray-Schauder continuation method. To do so, we show that the homotopy

$$
\begin{aligned}
(-\varepsilon, \varepsilon) & \times B(0, \varepsilon) \times[0,1] \ni(t, w, s) \longmapsto \\
& \left(\Phi(t, s w+\varphi(t)-\varphi(s t)), w-K w+\frac{Q N\left(s t e_{0}+s w\right)}{s}\right)
\end{aligned}
$$

is admissible on the set

$$
U_{\varepsilon}=\left\{u=t e_{0}+w \in X:|t|<\varepsilon,\|w\|<\varepsilon\right\},
$$

provided that $\varepsilon$ is sufficiently small. Here we have set

$$
\frac{Q N\left(s t e_{0}+s w\right)}{s}=0 \quad \text { for } s=0,
$$


according to the condition (8.2).

It suffices to show that the system of equations

$$
\begin{aligned}
& w-K w+\frac{Q N\left(s t e_{0}+s w\right)}{s}=0, \\
& \Phi(t, s w+\varphi(t)-\varphi(s t))=0
\end{aligned}
$$

has no solution for $0 \leq s \leq 1$ on the boundary

$$
\partial U=\{|t|=\varepsilon,\|w\| \leq \varepsilon\} \cup\{|t| \leq \varepsilon,\|w\|=\varepsilon\},
$$

if $\varepsilon$ is sufficiently small.

For each $|t| \leq \varepsilon$, the equation (8.10a) has a unique solution

$$
w=\frac{\varphi(s t)}{s} \quad \text { for } 0 \leq s \leq 1,
$$

where we set

$$
\frac{\varphi(s t)}{s}=0 \quad \text { for } s=0,
$$

recalling that $\varphi^{\prime}(0)=0$

(1) First, since we have the assertion

$$
\varphi(t)=o(t) \quad \text { as } t \rightarrow 0,
$$

we may choose $\varepsilon>0$ so small that

$$
\left\|\frac{\varphi(s t)}{s}\right\|=\left\|\frac{\varphi(s t)}{s t}\right\||t|<\varepsilon \quad \text { whenever }|t| \leq \varepsilon .
$$

This proves that the equation (8.10b) has no solution if $\|w\|=\varepsilon$ and $|t| \leq \varepsilon$. Indeed, we have the contradiction

$$
\|w\|=\left\|\frac{\varphi(s t)}{s}\right\|<\varepsilon=\|w\| .
$$

(2) Secondly, since we have the formula

$$
w=\frac{\varphi(s t)}{s} \quad \text { for } 0 \leq s \leq 1,
$$

then we have, by assertion (8.8),

$$
\Phi(t, s w+\varphi(t)-\varphi(s t))=\Phi(t, \varphi(t)) \neq 0 \quad \text { for }|t|=\varepsilon \text { and }\|w\| \leq \varepsilon .
$$

Summing up, we have proved that

$$
\Phi(t, s w+\varphi(t)-\varphi(s t)) \neq 0 \quad \text { if either }|t|=\varepsilon \text { or }\|w\|=\varepsilon .
$$

Namely, the system (8.10a) and (8.10b) has no solution on the boundary $\partial U$ for $0 \leq s \leq 1$.

Step 2: By Step 1, we obtain from Brown [22, Theorem 9.9] that $i(G, 0)$ is equal to the index of the mapping given by the formula

$$
(t, w) \longmapsto(\Phi(t, \varphi(t)), w-K w) .
$$


Hence we have, by the product formula ([17, Theorem 5.3.16], [22, Theorem 11.3]),

$$
i(G, 0)=i\left(\left.(I-K)\right|_{W}, 0\right) \cdot i(\Phi(\cdot, \varphi(\cdot)), 0) .
$$

However, since $\left.(I-K)\right|_{W}$ is invertible, it follows from formula (8.1) that

$$
i\left(\left.(I-K)\right|_{W}, 0\right)= \pm 1 .
$$

Furthermore, it follows from an application of Brown [22, Theorem 9.9] that

$$
i(\Phi(\cdot, \varphi(\cdot)), 0)=0, \pm 1
$$

since $\Phi(\cdot, \varphi(\cdot))$ maps a subset of $\mathbf{R}$ into $\mathbf{R}$.

By combining formulas (8.11), (8.12) and (8.13), we have proved the desired assertion

$$
|i(G, 0)| \leq 1 .
$$

The proof of Theorem 8.1 is now complete.

It is an easy consequence of the additivity property of degree that if $p$ is a regular value of $G$, that is, if $G^{-1}(p)$ contains no critical points, and if $\operatorname{deg}(G, U, p)=n$, then the nonlinear operator equation $G(u)=p$ has at least $|n|$ distinct solutions in $U$. This result can be extended as follows (see [52, Corollary 6.3]):

Corollary 8.1 Let $U$ be an open bounded subset of $X$ such that $p \notin G(\partial U)$. Assume that $\operatorname{deg}(G, U, p)=n$ and that

$$
\operatorname{dim} N\left(G^{\prime}(u)\right) \leq 1 \quad \text { for all } u \in G^{-1}(p) \cap U .
$$

Then the nonlinear operator equation $G(u)=p$ has at least $|n|$ distinct solutions in $U$.

Proof If not all of the solutions are isolated, there is nothing to prove. If all of the solutions are isolated, then the conclusion follows immediately from the additivity property of degree and Theorem 8.1 .

The proof of Corollary 8.1 is complete.

\subsection{Application to the resonance case}

In this subsection we apply Theorem 8.1 or Corollary 8.1 to the resonance case. More precisely, we consider the semilinear elliptic boundary value problem (1.2) with $\lambda:=\lambda_{1}$ :

$$
\begin{cases}A u-\lambda_{1} u+f(u)=0 & \text { in } D \\ B u=a\left(x^{\prime}\right) \frac{\partial u}{\partial \nu}+b\left(x^{\prime}\right) u=0 & \text { on } \partial D\end{cases}
$$

and prove that the semilinear problem (1.7) has at least two nontrivial solutions. form

To do so, we rewrite the semilinear problem (1.7) in the nonlinear operator

$$
G\left(u, \lambda_{1}\right)=u-\lambda_{1} K u+K(F(u))=0 .
$$

Recall that $\lambda_{1}$ is the first reciprocal eigenvalue of $K$ and further that $\lambda_{1}$ is algebraically simple:

$$
\lambda_{1} K e_{0}=e_{0} \quad \text { in } X=C(\bar{D}) .
$$

The next theorem is a generalization of Szulkin [52, Theorem 6.4] to the degenerate case: 
Theorem 8.2 Assume that hypotheses (H.1) and (H.2) are satisfied and let $f(t)$ be a $C^{1}$ function on $\mathbf{R}$ such that $f(0)=0$. Moreover, we assume that

$$
\lim _{t \rightarrow \pm \infty} f^{\prime}(t)=c
$$

and that

$$
0<c_{0} \leq \lambda_{1}-f^{\prime}(t) \leq c_{1}<\lambda_{2} .
$$

Then the nonlinear operator equation (8.14) has at least two nontrivial solutions $u_{1}$, $u_{2}$ if one of the following conditions is satisfied (see Figure 8.2):

(i) $f^{\prime}(0)<0<c$.

(ii) $c<0<f^{\prime}(0)$.

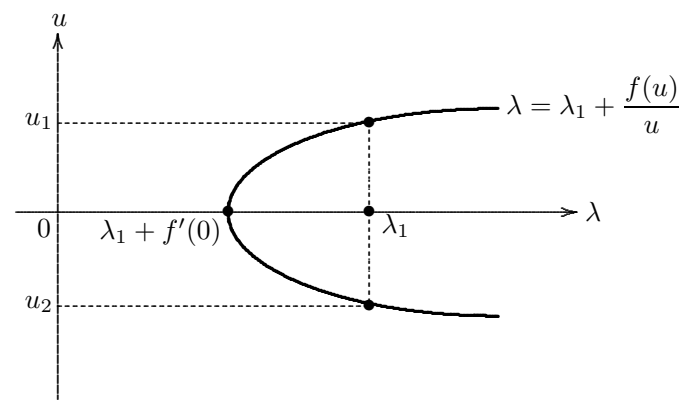

Fig. 8.2 The formal solution curve $\lambda=\lambda_{1}+\frac{f(u)}{u}$ in the case (i)

Proof The proof of Theorem 8.2 is divided into three steps.

Step 1: First, it follows from condition (8.14) that

$$
\mu_{2}\left(\lambda_{1}-f^{\prime}(u)\right)<\mu_{2}\left(\lambda_{2}\right)=1 .
$$

Hence we have the assertion

$$
\operatorname{dim} N\left(G_{u}\left(u, \lambda_{1}\right)\right) \leq 1 \quad \text { for any } u \in X .
$$

More precisely, we have the formula

$$
\operatorname{dim} N\left(G_{u}\left(u, \lambda_{1}\right)\right)= \begin{cases}1 & \text { if } \mu_{1}\left(\lambda_{1}-f^{\prime}(u)\right)=1 \\ 0 & \text { if } \mu_{1}\left(\lambda_{1}-f^{\prime}(u)\right) \neq 1\end{cases}
$$

By condition (8.15), we obtain that the mapping

$$
u: \longmapsto \lambda_{1} K u-K(F(u))
$$

is asymptotically linear and has the asymptote

$$
K\left(\lambda_{1}-c\right) \text {. }
$$


Step 2: We consider the case where condition (i) is satisfied. Then it follows that

$$
\mu_{1}\left(\lambda_{1}-c\right)=\frac{\lambda_{1}-c}{\lambda_{1}}<1
$$

Since we have the formula

$$
G\left(\cdot, \lambda_{1}\right)=I-\left(\lambda_{1} K-K(F(\cdot))\right)
$$

and assertions (8.17) and (8.18), we obtain from [29, Chapter 5, Proposition 5.8.11] that

$$
\operatorname{deg}\left(G\left(\cdot, \lambda_{1}\right), B(0, R), 0\right)=(-1)^{0}=1
$$

if the open ball $B(0, R)$ is sufficiently large. Indeed, it suffices to note that the operator $K\left(\lambda_{1}-c\right)$ has no eigenvalue greater than 1 .

On the other hand, we have, by condition (i) and condition (8.16),

$$
\mu_{1}\left(\lambda_{1}-f^{\prime}(0)\right)=\frac{\lambda_{1}-f^{\prime}(0)}{\lambda_{1}}>1
$$

and

$$
\mu_{2}\left(\lambda_{1}-f^{\prime}(0)\right)=\frac{\lambda_{1}-f^{\prime}(0)}{\lambda_{2}} \leq \frac{c_{1}}{\lambda_{2}}<1
$$

Therefore, by applying the Leray-Schauder index formula (3.5) (see Theorem 3.7) we obtain that

$$
i\left(G\left(\cdot, \lambda_{1}\right), 0\right)=(-1)^{1}=-1 .
$$

Indeed, it suffices to note that the operator

$$
K\left(\lambda_{1}-f^{\prime}(0)\right)
$$

has only one eigenvalue $\mu_{1}\left(\lambda_{1}-f^{\prime}(0)\right)$ greater than 1 .

Now we assume that all the solutions of the nonlinear operator equation $G\left(u, \lambda_{1}\right)=0$ are isolated. Then, we have, by formulas (8.19) and (8.20),

$$
\operatorname{deg}\left(G\left(\cdot, \lambda_{1}\right), B(0, R), 0\right)-i\left(G\left(\cdot, \lambda_{1}\right), 0\right)=2 .
$$

Therefore, by using Theorem 8.1 with

$$
\begin{aligned}
& C(u):=\lambda_{1} K u-K(F(u)), \\
& K:=\lambda_{1} K, \quad N(u):=K(F(u)),
\end{aligned}
$$

and the additivity property of degree we find that there must exist at least two additional nontrivial solutions $u_{1}, u_{2}$ of the nonlinear equation $G\left(u, \lambda_{1}\right)=0$ (see the proof of Corollary 8.1).

Step 3: Similarly, we can prove that if condition (ii) is satisfied, formula (8.21) holds true.

The proof of Theorem 8.2 is complete.

Results similar to our last theorem have been obtained in Ambrosetti-Mancini [9] and [10] by means of completely different methods.

For Theorem 8.2, we give a simple example of the nonlinear term $f(t)$ : 
Example 8.1 If $\lambda_{1}, \lambda_{2}$ are eigenvalues of the non-negative, selfadjoint operator $\mathfrak{A}$, we let

$$
f(t)=: \begin{cases}\frac{\lambda_{1}}{2} t+\frac{1}{2} \lambda_{1}-\lambda_{2} & \text { for } t>2, \\ \frac{\lambda_{1}}{2} t^{2}-\frac{\lambda_{1}+\lambda_{2}}{2} t+\frac{\lambda_{1}}{2} & \text { for } 1<t \leq 2, \\ \frac{\lambda_{1}-\lambda_{2}}{2} t & \text { for }-1 \leq t \leq 1, \\ -\frac{\lambda_{1}}{2} t^{2}-\frac{\lambda_{1}+\lambda_{2}}{2} t-\frac{\lambda_{1}}{2} & \text { for }-2 \leq t<-1, \\ \frac{\lambda_{1}}{2} t-\frac{\lambda_{1}}{2}+\lambda_{2} & \text { for } t<-2 .\end{cases}
$$

Then it is easy to verify that

$$
\begin{aligned}
& f^{\prime}(0)=\frac{\lambda_{1}-\lambda_{2}}{2}, \\
& c=\frac{\lambda_{1}}{2}, \quad c_{0}=\frac{\lambda_{1}}{2}, \quad c_{1}=\frac{\lambda_{1}+\lambda_{2}}{2} .
\end{aligned}
$$

\section{Concluding remarks}

This paper is devoted to the study of static bifurcation theory for a class of degenerate boundary value problems for semilinear elliptic differential operators of second-order, which includes as particular cases the Dirichlet and Robin problems. The purpose of this paper is to generalize some results of Szulkin [52] to the degenerate case. The approach here is distinguished by the extensive use of the ideas and techniques characteristic of the recent developments in the $L^{p}$ theory of pseudo-differential operators ([69, Part II $]$ ). In the proof of main theorems 1.2 , 1.3, 1.4 and 8.2 we re-work and expand in a different sprit the material of the previous papers [57] through [62] and [70], even though there is a lot of overlap in the contents of the present paper and those of them. This makes the present paper fairly self-contained.

This paper will provide a solid foundation for the reader interested in elliptic boundary value problems and topological methods in nonlinear analysis such as the bifurcation theory from a simple eigenvalue due to Crandall-Rabinowitz [26] and Dancer [27] and the Leray-Schauder degree introduced by Leray-Schauder [39], via the Semenov approximation in Chemistry.

For the semilinear elliptic boundary value problem (1.2), we give an overview of the classical Schauder theory in Szulkin [52] versus the theory of pseudo-differential operators in the present paper (see Table 9.1 below).

Finally, it should be noticed that Anh-My [14] studied semilinear Dirichlet problems for a strongly degenerate elliptic system of Hamiltonian type (see [34]) and further that Papageorgiou-Zhang [45] studied nonlinear Dirichlet problems for the sum of a $p$-Laplacian and a Laplacian with concave terms (see [41]).

\section{Appendix: The maximum principle in $L^{p}$ Sobolev spaces}

In this appendix we formulate various maximum principles for second-order, elliptic differential operators with discontinuous coefficients such as the weak and strong maximum principles (Theorems A.1 and A.2) and the Hopf boundary point 


\begin{tabular}{|c|c|c|}
\hline $\begin{array}{c}\text { Type of } \\
\text { nonlinearity }\end{array}$ & $\begin{array}{c}\text { Classical } \\
\text { Schauder theory }\end{array}$ & $\begin{array}{c}\text { Theory of } \\
\text { pseudo-differential operators }\end{array}$ \\
\hline \hline $\begin{array}{c}\text { Asymptotically linear } \\
\text { nonlinearity case }\end{array}$ & {$[52$, Theorem 1.3$]$} & Theorem 1.2 \\
\hline $\begin{array}{c}\text { Bounded nonlinearity } \\
\text { case }\end{array}$ & {$[52$, Theorem 1.4$]$} & Corollary 1.1 \\
\hline$[52$, Theorem 5.2$]$ & Theorem 1.3 \\
Theorem 1.4
\end{tabular}

Table 9.1 An overview of the classical Schauder theory versus the theory of pseudo-differential operators for the semilinear elliptic boundary value problem (1.2)

lemma (Lemma A.1) in the framework of $L^{p}$ Sobolev spaces. The results here are adapted from Bony [20], Troianiello [72, Chapter 3] and also Taira [64, Chapter 8].

Let $D$ be a bounded domain in Euclidean space $\mathbf{R}^{N}, N \geq 3$, with boundary $\partial D$ of class $C^{1,1}$. We consider a second-order, uniformly elliptic differential operator $A$ with real discontinuous coefficients in non-divergence form

$$
A u:=-\sum_{i, j=1}^{N} a^{i j}(x) \frac{\partial^{2} u}{\partial x_{i} \partial x_{j}}+\sum_{i=1}^{N} b^{i}(x) \frac{\partial u}{\partial x_{i}}+c(x) u .
$$

More precisely, we assume that the coefficients $a^{i j}(x), b^{i}(x)$ and $c(x)$ of the differential operator $A$ satisfy the following three conditions:

(1) $a^{i j}(x) \in L^{\infty}(D), a^{i j}(x)=a^{j i}(x)$ for almost all $x \in D$ and there exist a constant $\lambda>0$ such that

$$
\frac{1}{\lambda}|\xi|^{2} \leq \sum_{i, j=1}^{N} a^{i j}(x) \xi_{i} \xi_{j} \leq \lambda|\xi|^{2} \quad \text { for almost all } x \in D \text { and all } \xi \in \mathbf{R}^{N} .
$$

(2) $b^{i}(x) \in L^{\infty}(D)$ for all $1 \leq i \leq N$.

(3) $c(x) \in L^{\infty}(D)$ and $c(x) \geq 0$ for almost all $x \in D$.

First, we state a variant of the weak maximum principle in the framework of $L^{p}$ Sobolev spaces, due to Bony [20] ([72, Chapter 3, Lemma 3.25]):

Theorem A.1 (the weak maximum principle) If a function $u \in W^{2, p}(D), N<$ $p<\infty$, satisfies the condition

$$
A u(x) \leq 0 \quad \text { for almost all } x \in D,
$$

then we have the inequality

$$
\max _{\bar{D}} u \leq \max _{\partial D} u^{+}
$$


where

$$
u^{+}(x)=\max \{u(x), 0\} \quad \text { for } x \in \bar{D} .
$$

A detailed proof of Theorem A.1 is given in Taira [67, Theorem 8.1].

Secondly, the Hopf boundary point lemma reads as follows ([72, Chapter 3, Lemma 3.26], [68, Lemma 6.1]):

Lemma A.1 (the boundary point lemma) Assume that a function $u \in W^{2, p}(D)$, $N<p<\infty$, satisfies the condition

$$
A u(x) \leq 0 \quad \text { for almost all } x \in D .
$$

If $u(x)$ attains a non-negative, strict local maximum at a point $x_{0}^{\prime}$ of $\partial D$, then we have the inequality

$$
\frac{\partial u}{\partial \boldsymbol{\nu}}\left(x_{0}^{\prime}\right)>0
$$

(see Figure 1.1).

Finally, we can obtain the following strong maximum principle for the operator $A$ ([20, Théorème 2], [72, Chapter 3, Theorem 3.27], [68, Theorem 6.2]):

Theorem A.2 (the strong maximum principle) Assume that a function $u \in$ $W^{2, p}(D), N<p<\infty$, satisfies the condition

$$
A u(x) \leq 0 \quad \text { for almost all } x \in D
$$

If $u(x)$ attains a non-negative maximum at an interior point $x_{0}$ of $D$, then it is a (non-negative) constant function.

Acknowledgements I am grateful to the referees for their valuable suggestions and for informing me of additional references, which improved the presentation of this paper.

\section{References}

1. Adams, R. A. and Fournier, J. J. F.: Sobolev spaces, second edition. Pure and Applied Mathematics, vol. 140. Elsevier/Academic Press, Amsterdam Heidelberg New York Oxford (2003)

2. Agmon, S.: Lectures on elliptic boundary value problems. Van Nostrand, Princeton, New Jersey (1965)

3. Agmon, S., Douglis, A. and Nirenberg, L.: Estimates near the boundary for solutions of elliptic partial differential equations satisfying general boundary conditions I. Comm. Pure Appl. Math. 12, 623-727 (1959)

4. Amann, H.: On the number of solutions of nonlinear equations in ordered Banach spaces. J. Functional Analysis 11, 346-384 (1972)

5. Amann, H.: Fixed point equations and nonlinear eigenvalue problems in ordered Banach spaces. SIAM Rev. 18, 620-709 (1976)

6. Amann, H.: Nonlinear eigenvalue problems having precisely two solutions. Math. Z. 150, 27-37 (1976)

7. Amann, H., Ambrosetti, A, and Mancini, G.: Elliptic equations with noninvertible Fredholm linear part and bounded nonlinearities. Math. Z., 158, 179-194 (1978)

8. Ambrosetti, A. and Malchiodi, A.: Nonlinear analysis and semilinear elliptic problems. Cambridge Studies in Advanced Mathematics, no. 104, Cambridge University Press, Cambridge (2007) 
9. Ambrosetti, A. and Mancini, G.: Existence and multiplicity results for nonlinear elliptic problems with linear part at resonance. The case of the simple eigenvalue. J. Differential Equations 28, 220-245 (1978)

10. Ambrosetti, A. and Mancini, G.: Theorems of existence and multiplicity for nonlinear elliptic problems with noninvertible linear part. Ann. Scuola Norm. Sup. Pisa, Cl. Sci. (4) 5, 15-28 (1978)

11. Ambrosetti, A. and Mancini, G.: Sharp nonuniqueness results for some nonlinear problems. Nonlinear Analysis TMA 3, 635-645 (1979)

12. Ambrosetti, A. and Prodi, G.: On the inversion of some differentiable mappings with singularities between Banach spaces. Ann. Mat. Pura Appl. 93, 231-247 (1972)

13. Ambrosetti, A. and Prodi, G.: A primer of nonlinear analysis, paperback edition (with corrections). Cambridge Studies in Advanced Mathematics, no. 34. Cambridge University Press, Cambridge (1993)

14. Anh, C. T. and My, B. K.: Existence and non-existence of solutions to a Hamiltonian strongly degenerate elliptic system. Adv. Nonlinear Anal. 8, 661-678 (2019)

15. Aronszajn, N., Krzywicki, A. and Szarski, J.: A unique continuation theorem for exterior differential forms on Riemannian manifolds. Ark. Mat. 4, 417-453 (1962)

16. Berestycki, H. : Remarques sur le nombre de solutions de certains problèmes semi-linéaires elliptiques. C. R. Acad. Sci. Paris Sér. A-B 289, A633-A636 (1979)

17. Berger, M. S.: Nonlinearity and Functional Analysis. Academic Press, New York (1977)

18. Berger, M. S.: Nonlinear problems with exactly three solutions. Indiana Univ. Math. J. 28, 689-698 (1979)

19. Bergh, L. and Löfström, J.: Interpolation spaces, an introduction. Springer-Verlag, Berlin Heidelberg New York (1976)

20. Bony, J.-M.: Principe du maximum dans les espaces de Sobolev. C. R. Acad. Sc. Paris Sér. A-B 265, A333-A336 (1967)

21. Bourdaud, G.: $L^{p}$-estimates for certain non-regular pseudo-differential operators. Comm. Partial Differential Equations 7, 1023-1033 (1982)

22. Brown, R. F.: A topological introduction to nonlinear analysis, third edition. Springer, Cham (2014)

23. Chang, K.-C.: Methods in nonlinear analysis. Springer Monographs in Mathematics Springer-Verlag, Berlin (2005)

24. Chazarain, J. et Piriou, A.: Introduction à la théorie des équations aux dérivées partielles linéaires. Gauthier-Villars, Paris (1981)

25. Chow, S. N. and Hale, J. K.: Methods of bifurcation theory. Springer-Verlag, New York Heidelberg Berlin (1982)

26. Crandall, M. G. and Rabinowitz, P. H.: Bifurcation from simple eigenvalues. J. Functional Analysis 8, 321-340 (1971)

27. Dancer, E. N.: Global solution branches for positive mappings. Arch. Rational Mech. Anal., 52, 181-192 (1973)

28. de Figueiredo, D. G.: Positive solutions of semilinear elliptic problems. In: Differential equations, Lecture Notes in Mathematics, no. 957, pp. 34-87, Springer-Verlag, Berlin Heidelberg New York (1982)

29. Drábek, P. and Milota, J.: Methods of nonlinear analysis, Applications to differential equations, second edition. Birkhäuser Advanced Texts: Basler Lehrbücher. Birkhäuser/Springer Basel AG, Basel (2013)

30. Gilbarg, D. and Trudinger, N. S.: Elliptic partial differential equations of second order, reprint of the 1998 edition. Classics in Mathematics, Springer-Verlag, New York Berlin Heidelberg Tokyo (2001)

31. Hörmander, L.: The analysis of linear partial differential operators III, 1994 edition. Grundlehren Math. Wiss., Springer-Verlag, Berlin (1994)

32. Iwasaki, C.: The asymptotic expansion of the fundamental solution for parabolic initialboundary value problems and its application. Osaka J. Math. 31, 663-728 (1994)

33. Kazdan, J. L.: Unique continuation in geometry. Comm. Pure Appl. Math. 41, 667-681 (1988)

34. Kogoj, A. and Lanconelli, E.: On semilinear $\Delta_{\lambda}$-Laplace equation. Nonlinear Analysis TMA 75, 4637-4649 (2012)

35. Kohn, J. J. and Nirenberg, L.: Non-coercive boundary value problems. Comm. Pure Appl. Math. 18, 443-492 (1965)

36. Krasnosel'skii, M. A.: Positive solutions of operator equations. P. Noordhoff, Groningen (1964) 
37. Kreı̆n, M. G. and Rutman, M. A.: Linear operators leaving invariant a cone in a Banach space. Amer. Math. Soc. Transl. 10, 199-325 (1962)

38. Landesman, E. A. and Lazer, A. C.: Nonlinear perturbations of linear elliptic boundary value problems at resonance. J. Math. Mech. 19, 609-623 (1970)

39. Leray, J. et Schauder, J.: Topologie et équations fonctionelles. Ann. Sci. École Norm. Sup. 51, 45-78 (1934)

40. Lyapunov, A. M.: The general problem of the stability of motion, Translated by A. T. Fuller from Édouard Davaux's French translation (1907) of the 1892 Russian original. With an editorial (historical introduction) by Fuller, a biography of Lyapunov by V. I. Smirnov, and the bibliography of Lyapunov's works collected by J. F. Barrett. Lyapunov centenary issue. Internat. J. Control, 55, 531-773 (1992)

41. Marano, S. A. and Mosconi, S. J. N.: Some recent results on the Dirichlet problem for $(p, q)$-Laplace equations. Discrete Contin. Dyn. Syst. Ser. S 11, 279-291 (2018)

42. Miranda, C.: Partial Differential Equations of Elliptic Type, second revised edition. Springer-Verlag, New York Berlin (1970)

43. Nirenberg, L.: Topics in nonlinear functional analysis, revised reprint of the 1974 original. Courant Lecture Notes in Mathematics, No, 6. New York University, Courant Institute of Mathematical Sciences, New York; American Mathematical Society, Providence, Rhode Island (2001)

44. Papageorgiou, N. S., Rădulescu, V. D. and Repovš, D. D.: Nonlinear analysis - theory and methods. Springer Monographs in Mathematics, Springer, Cham (2019)

45. Papageorgiou, N. S. and Zhang, C.: Noncoercive resonant $(p, 2)$-equations with concave terms. Adv. Nonlinear Anal. 9, 228-249 (2020)

46. Plastock, R. A.: Nonlinear Fredholm maps of index zero and their singularities. Proc. Amer. Math. Soc. 68, 317-322 (1978)

47. Rabinowitz, P. H.: Some global results for nonlinear eigenvalue problems. J. Functional Analysis 7, 487-513 (1971)

48. Reed, M. and Simon, B.: Methods of modern mathematical physics, IV: Analysis of operators. Academic Press, San Diego New York Boston London Sydney Tokyo Toronto (1978)

49. Runst, T. and Sickel, W.: Sobolev spaces of fractional order, Nemytskij operators, and nonlinear partial differential equations. Walter de Gruyter, Berlin New York (1996)

50. Schmidt, E.: Zur Theorie der linearen und nichtlinearen Integralgleichungen, III. Teil. Über die Auflösung der nichtlinearen Integralgleichung und die Verzweigung ihrer Lösongen. Math. Ann. 65, 370-399 (1908)

51. Semenov, N. N.: Chemical kinetics and chain reactions. Clarendon Press, Oxford (1935)

52. Szulkin, A.: On the number of solutions of some semilinear elliptic boundary value problems. Nonlinear Analysis TMA 6, 95-116 (1982)

53. Taira, K.: On some degenerate oblique derivative problems. J. Fac. Sci. Univ. Tokyo Sec. IA Math. 23, 259-287 (1976)

54. Taira, K.: Boundary value problems for elliptic integro-differential operators. Math. Z. 222, 305-327 (1996)

55. Taira, K.: Bifurcation for nonlinear elliptic boundary value problems I. Collect. Math. 47, 207-229 (1996)

56. Taira, K.: Diffusive logistic equations with degenerate boundary conditions. Mediterr. J. Math. 1, 315-365 (2004)

57. Taira, K.: Degenerate elliptic eigenvalue problems with indefinite weights. Mediterr. J. Math. 5, 133-162 (2008)

58. Taira, K.: Degenerate elliptic boundary value problems with asymmetric nonlinearity. J. Math. Soc. Japan 62, 431-465 (2010)

59. Taira, K.: Multiple solutions of semilinear degenerate elliptic boundary value problems. Math. Nachr., 284, 1-19 (2011)

60. Taira, K.: Multiple solutions of semilinear degenerate elliptic boundary value problems II. Math. Nachr. 284, 1554-1566 (2011)

61. Taira, K.: Degenerate elliptic boundary value problems with asymptotically linear nonlinearity. Rend. Circ. Mat. Palermo (2) 60, 283-308 (2011)

62. Taira, K.: Multiple solutions of semilinear elliptic problems with degenerate boundary conditions. Mediterr. J. Math. 10, 731-752 (2013)

63. Taira, K.: A mixed problem of linear elastodynamics. J. Evol. Equ. 13, 481-507 (2013)

64. Taira, K.: Semigroups, boundary value problems and Markov processes, second edition. Springer Monographs in Mathematics, Springer-Verlag, Berlin Heidelberg New York (2014) 
65. Taira, K.: Analytic semigroups and semilinear initial-boundary value problems, second edition. London Mathematical Society Lecture Note Series, vol. 434. Cambridge University Press, Cambridge (2016)

66. Taira, K.: Spectral analysis of the hypoelliptic Robin problem. Ann. Univ. Ferrara Sez. VII Sci. Mat. 65, 171-199 (2019)

67. Taira, K.: Dirichlet problems with discontinuous coefficients and Feller semigroups. Rend. Circ. Mat. Palermo (2) 69, 287-323 (2020)

68. Taira, K.: Ventcel' boundary value problems for elliptic Waldenfels operators. Boll. Unione Mat. Ital. 13, 213-256 (2020)

69. Taira, K.: Boundary value problems and Markov processes: Functional analysis methods for Markov processes, third edition. Lecture Notes in Mathematics, no. 1499, SpringerVerlag, Berlin Heidelberg New York (2020)

70. Taira, K. and Umezu, K.: Bifurcation for nonlinear elliptic boundary value problems II. Tokyo J. Math. 19, 387-396 (1996)

71. Triebel, H.: Theory of function spaces II. Monographs in Mathematics, vol. 84. Birkhäuser, Basel (1992)

72. Troianiello, G. M.: Elliptic differential equations and obstacle problems. The University Series in Mathematics. Plenum Press, New York London (1987)

73. Wloka, J.: Partial differential equations. Cambridge University Press, Cambridge (1987)

74. Yosida, K.: Functional analysis, reprint of the sixth (1980) edition. Classics in Mathematics, Springer-Verlag, Berlin Heidelberg New York (1980)

75. Zeidler, E.: Nonlinear functional analysis and its applications I. Springer-Verlag, New York Berlin Heidelberg (1986) 